\title{
British Thoracic Society guideline for diagnostic flexible bronchoscopy in adults
}

\author{
I A Du Rand, ${ }^{1}$ J Blaikley, ${ }^{2}$ R Booton, ${ }^{3} \mathrm{~N}$ Chaudhuri, ${ }^{4}$ V Gupta, ${ }^{2}$ S Khalid, ${ }^{5}$ S Mandal, ${ }^{6}$ \\ J Martin, ${ }^{4}$ J Mills, ${ }^{7}$ N Navani, ${ }^{8}$ N M Rahman, ${ }^{9}$ J M Wrightson, ${ }^{9}$ M Munavvar, ${ }^{7}$ \\ on behalf of the British Thoracic Society Bronchoscopy Guideline Group
}

- Additional material is published online only. To view please visit the journal online (http://dx.doi.org/10.1136/ thoraxjnl-2013-203618).

${ }^{1}$ Worcestershire Royal Hospital, Worcestershire Acute Hospitals NHS Trust, Worcester, UK

${ }^{2}$ The University of Manchester, Manchester, UK

${ }^{3}$ The University of Manchester, Manchester Academic Health Science Centre, University Hospital South Manchester NHS Foundation Trust, Manchester, UK

${ }^{4}$ University Hospital of South Manchester, Manchester, UK ${ }^{5}$ Royal Blackburn Hospital, Lancashire, UK

'Lane Fox Unit, St Thomas' Hospital, London, UK

${ }^{7}$ Lancashire Teaching Hospitals NHS Trust, Preston, UK ${ }^{8}$ University College London Hospital and MRC Clinical Trials Unit, National Institute for Health Research University College London Hospitals Biomedical Research Centre, London, UK

${ }^{9}$ Oxford Centre for Respiratory Medicine, NIHR Oxford Biomedical Research Centre, Oxford Respiratory Trials Unit, University of Oxford, Oxford, UK

\section{Correspondence to} Dr Ingrid Du Rand,

Worcestershire Royal Hospital, Aconbury East, Charles Hastings Way, Worcester, WR5 1DD, UK;

ingrid.durand@nhs.net

\section{SUMMARY OF RECOMMENDATIONS}

Monitoring, precautions and complications

- All patients undergoing bronchoscopy should have heart rate, respiratory rate, blood pressure and oxygen saturation recorded repeatedly, including before, during and after the procedure. (Grade D)

- All bronchoscopy units should undertake periodic audit of bronchoscopic performance, including efficacy, complications and patient satisfaction surveys. (Good practice point $(\sqrt{ })$ )

- All Trusts should have a 'safe sedation policy', and ensure all bronchoscopy unit staff, including trainees, receive appropriate training. $(\sqrt{ })$

\section{Hypoxaemia}

- Patients should be monitored by continuous pulse oximetry during bronchoscopy. (Grade C)

- Oxygen supplementation should be used when desaturation is significant (pulse oximeter oxygen saturation $\left(\mathrm{SpO}_{2}\right)>4 \%$ change, or $\left.\mathrm{SpO}_{2}<90 \%\right)$ and prolonged $(>1 \mathrm{~min})$ to reduce the risk of hypoxaemia-related complications. (Grade D)

- The risks of hypoxaemia-related complications are associated with baseline arterial oxygen saturation $\left(\mathrm{SaO}_{2}\right)$ and lung function, comorbidity, sedation and procedural sampling. Fitness for bronchoscopy should incorporate an assessment of these elements, and appropriate monitoring and preprocedure optimisation. (Grade D)

\section{Cardiac arrhythmias}

- Continuous ECG monitoring should be used when there is a high clinical risk of arrhythmia. (Grade D)

- When there is a high risk of arrhythmia, oxygen saturations, pulse rate and blood pressure should be optimised. Appropriate aftercare monitoring and instructions should be given. (Grade D)

- Resuscitation equipment should be readily available. $(\sqrt{ })$

- Intravenous access should be established before sedation is given and maintained until discharge. $(\sqrt{ })$

\section{Bleeding complications}

- Perform coagulation studies, platelet count and haemoglobin concentration when there are clinical risk factors for abnormal coagulation. (Grade D)

- Bronchoscopy with lavage can be performed with platelet counts $>20000$ per $\mu \mathrm{L}$. Liaise with the local haematology team regarding the need for platelet transfusion before bronchoscopy if endobronchial biopsy (EBB) or transbronchial lung biopsy (TBLB) is planned. (Grade D)

- Discontinue clopidogrel 7 days prior to consideration of EBB and TBLB. Low-dose aspirin alone can be continued. (Grade C)

- Anticoagulants should be managed according to published guidelines as set out in appendix 7 of this guideline. $(\sqrt{ })$

- The risk of biopsy needs to be weighed against the potential for benefit and appropriate informed consent obtained. $(\sqrt{ })$

\section{Pneumothorax}

- A chest radiograph should be obtained if a patient is symptomatic or there is a clinical suspicion of possible pneumothorax after TBLB. (Grade D)

- Fluoroscopic screening may improve diagnostic yield of TBLB in focal but not diffuse lung disease. (Grade D)

- Patients should be advised of the potential for delayed complications following TBLB and provided with written information regarding likely symptoms and action required. (Grade D)

\section{Fever and infection}

- Patients should receive written information regarding post-bronchoscopy fever (PBF) and appropriate management advice. (Grade $\mathrm{C}$ )

- Antibiotic prophylaxis is not warranted before bronchoscopy for the prevention of endocarditis, fever or pneumonia. (Grade B)

\section{SAFETY OF FLEXIBLE BRONCHOSCOPY IN SPECIFIC MEDICAL CONDITIONS \\ Asthma}

- Patients' asthma control should be optimised prior to bronchoscopy, especially when bronchoalveolar lavage (BAL) is likely to be performed. (Grade C)

- Nebulised bronchodilators should be considered before bronchoscopy in patients with asthma. $(\sqrt{ })$

\section{Chronic obstructive pulmonary disease}

- Chronic obstructive pulmonary disease (COPD) treatment should be optimised prior to bronchoscopy when possible. (Grade D)

- Bronchoscopists should be cautious when sedating patients with COPD. (Grade D)

\section{Ischaemic heart disease}

- Liaison with cardiologists should be considered in high-risk patients with cardiac disease and if 
flexible bronchoscopy (FB) is indicated within 4-6 weeks after myocardial infarction (MI). (Grade D)

- FB should ideally be delayed for 4 weeks after MI. (Grade D)

\section{Haemoptysis}

- Consider bronchoscopy after a normal CT if the patient is high risk for lung carcinoma or if the haemoptysis continues. (Grade D)

\section{Older patients}

- Age alone should not be a contraindication for bronchoscopy. (Grade D)

- The older patient may require reduced doses of benzodiazepines/opioids sedation. (Grade D)

\section{Patients who are immunosuppressed}

- When a diagnosis is not likely to be obtained through noninvasive measures, bronchoscopy with BAL can be considered to provide diagnostic information. (Grade C)

- TBLB is helpful in lung transplant recipients when rejection is a possibility. (Grade C)

\section{SEDATION}

\section{Premedication}

- Anticholinergics (glycopyrrolate or atropine) should not routinely be used prior to bronchoscopy due to a lack of clinical benefit and a possible increased risk of haemodynamic changes. (Grade A)

- Premedication for bronchoscopy is not routinely indicated. (Grade C)

\section{Sedation}

- Intravenous sedation should be offered to patients undergoing bronchoscopy, provided there are no contraindications. (Grade B)

- Some patients will tolerate unsedated bronchoscopy well, and patient preference should be sought. (Grade B)

- Sedative drugs should be titrated to provide the desired depth of sedation, given significant inter-patient variability in required doses. (Grade B)

- The desired depth of sedation is one in which verbal contact is possible at all times. (Grade D)

- Bronchoscopists are encouraged to document an assessment of sedation depth as part of the procedural report. $(\sqrt{ })$

\section{Benzodiazepines}

- Intravenous midazolam is the preferred drug for sedation, having a rapid onset of action, being titratable to provide the required depth of sedation, and being reversible. (Grade B)

- No more than $5 \mathrm{mg}$ midazolam should be initially drawn up into any syringe prior to bronchoscopy for patients under the age of 70 (2 mg midazolam for patients over 70 ) to prevent potential inadvertent oversedation associated with the practice of routinely drawing up $10 \mathrm{mg}$ midazolam. (Grade D)

- Only low-strength midazolam $(1 \mathrm{mg} / \mathrm{mL})$ should be available within bronchoscopy suites. High-strength midazolam (2 mg/ $\mathrm{mL}$ or $5 \mathrm{mg} / \mathrm{mL}$ ) should be restricted to general anaesthesia, intensive care and other areas where its use has been formally risk assessed. (Grade D)

\section{Propofol}

- While propofol has similar efficacy to midazolam, it should only be used when administered by practitioners formally trained in its administration (eg, anaesthetists) since it has a narrow therapeutic window beyond which general anaesthesia is achieved. (Grade B)

\section{Opioids}

- Combination opioid and midazolam sedation should be considered in patients to improve bronchoscopic tolerance. (Grade B)

- When opioids are used, short-acting agents (such as fentanyl or alfentanil) should be used to minimise post-procedural sedation. (Grade D)

- When combination sedatives are used, opioids should be administered first and allowed time to become maximally effective before administration of any other agent. (Grade D)

\section{Topical anaesthesia}

- Lidocaine should be used for topical anaesthesia during bronchoscopy, unless contraindicated. (Grade A)

- Nasal topical anaesthesia is most effectively provided using 2\% lidocaine gel. (Grade A)

- Both cricothyroid and spray-as-you-go techniques are effective in delivering lidocaine to the vocal cords and trachea. (Grade B)

- Nebulisation is not recommended as a technique for delivering lidocaine to the airways. (Grade B)

- $1 \%$ lidocaine solution should be used for spray-as-you-go administration. (Grade A)

- To reduce the risk of lidocaine toxicity, bronchoscopists should use the lowest dose of lidocaine sufficient to prevent excessive coughing and provide patient comfort. (Grade D)

- Bronchoscopists should remain vigilant for objective and subjective symptoms of lidocaine toxicity, particularly given significant inter-patient variability in lidocaine absorption and metabolism. (Grade B)

- Bronchoscopists should monitor and document the total lidocaine dose delivered at all sites during bronchoscopy. $(\sqrt{ })$

\section{Sampling and diagnostic accuracy}

- Bronchoscopists should maintain a record of their personal diagnostic accuracy for FB. $(\sqrt{ })$

\section{Lung cancer}

- A diagnostic level of $85 \%$ should be attainable when definite endobronchial tumour is visible. (Grade B)

- At least five biopsy samples should be taken when endobronchial tumour is visible to maximise diagnostic yield and the volume of biopsy tissue and to allow for tumour phenotyping and genotyping. (Grade D)

- When endobronchial tumour is visible, brushings and washings can increase the diagnostic yield of the procedure. (Grade D)

- A chest CT scan should be performed prior to a diagnostic bronchoscopy in patients with suspected lung cancer. (Grade D)

\section{Interstitial lung disease}

- In suspected sarcoidosis, EBBs should be considered to increase the diagnostic yield. (Grade C)

- TBLB is recommended for the diagnosis of stage II-IV sarcoidosis. (Grade C)

- In patients with diffuse interstitial lung disease (ILD), five to six TBLBs should be taken from the same lung. (Grade D) 
- Fluoroscopy should be considered for TBLB in patients with localised or focal parenchymal lung disease. (Grade D)

\section{Diagnosis of infection}

\section{Patients who are immunocompromised}

- In patients with pulmonary infiltrates who are immunocompromised and in whom tuberculosis (TB) is considered unlikely, BAL alone is usually sufficient to achieve a diagnosis. In areas or populations with high prevalence of $\mathrm{TB}$, TBLB may be considered in addition. (Grade C)

- BAL or bronchial washings should be sent for microscopy for acid fast bacteria (AFB) and for mycobacterial culture in patients with pneumonia who are immunocompromised. (Grade C)

- Post-bronchoscopy sputum could be collected in patients who are immunocompromised and suspected to have TB. (Grade D)

- TBLB and EBB for invasive aspergillosis may be avoided if BAL galactomannan test is available due to the high sensitivity and specificity of the latter and inherent risks with the biopsies. (Grade C)

- In patients suspected to have invasive aspergillosis, BAL should be sent for microscopy for hyphae and fungal culture; a BAL galactomannan test should be considered to further improve diagnostic yield. (Grade C)

\section{Patients who are immunocompetent}

- Bronchoscopy may be considered in patients with non-resolving or slowly resolving pneumonia, especially if they are current or ex smokers and older than 50 years. (Grade C)

- If bronchoscopy is performed for community-acquired pneumonia, BAL specimens should be sent for legionella PCR and atypical pathogens. (Grade $\mathrm{C}$ )

- Bronchoscopy may be considered if the patient is suspected to have TB when sputum smear is negative. (Grade $\mathrm{C}$ )

- In cases of suspected TB, BAL, bronchial aspirates and postbronchoscopy sputum appear to be complementary and should all be analysed. (Grade C)

- In areas with high or intermediate prevalence of TB, patients undergoing bronchoscopy for another indication should have samples sent routinely for cultures for TB. (Grade C)

\section{INTENSIVE CARE UNITS}

- The external diameter of a bronchoscope used in the intensive care unit (ICU) setting should be carefully selected according to the external diameter of the bronchoscope, the size of the airway support device (endotracheal tube (ET) or laryngeal mask) and the type of airway device used. (Grade D)

- Prophylactic bronchoscopy and lavage should not be used to prevent post-lobectomy atelectasis in ventilated patients. (Grade A)

- Bronchoscopy may be considered in specific circumstances for the relief of atelectasis in intubated and ventilated patients. (Grade D)

- Bronchoscopy may be considered in ventilated patients with haemoptysis if CT imaging has been performed and is unhelpful, or is not possible. (Grade D)

- Directed non-invasive diagnostic strategies (eg, blind catheter aspiration) should be used first line in preference to bronchoscopy in ventilated patients with suspected ventilatorassociated pneumonia. (Grade A)

- When such non-invasive diagnostic techniques fail to identify a responsible organism, bronchoscopy should be considered for the diagnosis of ventilator-associated pneumonia. (Grade D)
- Patients in the ICU should be considered at high risk from complications when undergoing bronchoscopy. (Grade D)

- All potential risk factors (ventilator parameters, clotting dysfunction) should be corrected as far as possible before undertaking bronchoscopy. (Grade D)

- The risks and benefits of bronchoscopy should be carefully considered in mechanically ventilated patients. $(\sqrt{ })$

- Continuous multimodal physiological monitoring should occur during and after bronchoscopy in the ICU setting. (Grade C)

- Patients should be monitored after the procedure for complications, including pneumothorax, even when a biopsy has not been taken. (Grade D)

- Continuous positive airway pressure (CPAP) plus oxygen support may be considered in patients with hypoxia undergoing bronchoscopy to prevent desaturation and postprocedure requirement for mechanical ventilation. (Grade B)

- When patients require non-invasive ventilation prior to bronchoscopy, the procedure should be conducted in an environment where intubation and ventilatory support are readily accessible. (Grade D)

- Bronchoscopy should be undertaken cautiously in patients with documented or suspected raised intracranial pressure (ICP). (Grade D)

- Care must be exercised to ensure adequate ventilation and oxygenation is maintained during bronchoscopy in intubated patients. $(\sqrt{ })$

- Adequate sedation and analgesia should be provided for patients undergoing bronchoscopy in an intensive care setting. The risks of these procedures should be carefully balanced with their potential benefit in ventilated patients. (Grade D)

- Clinicians administering sedation/anaesthesia/analgesia should be acquainted with the use of these agents, and the anaesthetist/intensivist is usually best placed to fulfil this role. (Grade D)

\section{DISINFECTION}

- All personnel involved in cleaning and decontaminating bronchoscopes must receive specific training in infection control practices and decontamination processes. (Grade D)

- Decontamination and disinfection should be carried out at the beginning and end of each list and after each patient use. If drying cabinets or storage chambers are unavailable bronchoscopes should be decontaminated no more than $3 \mathrm{~h}$ before the procedure to eliminate colonisation of pathogens. (Grade D)

- Bronchoscopes should be cleaned in designated cleaning areas. Used scopes must be separated from clean scopes to prevent cross contamination. (Grade D)

- Thorough cleaning, brushing and flushing of all accessible channels with enzymatic or low foaming detergent remains the most important initial stage of the cleaning process. (Grade D)

- Single-use suction valves should replace reusable valves wherever possible. Single-use valves must be discarded after each procedure. (Grade D)

- Reusable valves should be used only with one bronchoscope and stored alongside the scope for traceability. (Grade D)

- Single-use accessories should be selected over reusable accessories wherever possible. (Grade D)

- When it is necessary to use reusable accessories they must be cleaned according to the manufacturer's recommendations. (Grade D) 
- Tracking of patient use of equipment and cleaning processes must be completed after each use. (Grade D)

- On the grounds of staff safety, manual disinfection is no longer recommended. (Grade D)

- Bronchoscopes should be processed in automated endoscope reprocessors (AERs). (Grade D)

- Aldehyde-based disinfectants are no longer recommended. (Grade C)

- Alternative, recommended disinfectants should be used in accordance with the manufacturer's instructions. (Grade D)

- Disinfectant times should be those recommended by disinfectant manufacturers. (Grade D)

- Universal decontamination procedures should be performed before and after all procedures to avoid transmission of HIV. (Grade D)

- The use of 70\% alcohol after final rinse is no longer recommended as it is considered to act as a fixative. (Grade D)

- Drying cabinets/storage chambers are recommended for storing clean bronchoscopes. Compatibility of bronchoscopes must be confirmed with individual instrument manufacturers. (Grade D)

- Bronchoscopes stored in drying cabinets or storage chambers should be reprocessed in accordance with the manufacturer's recommendations. (Grade D)

- When drying cabinets or storage chambers are not available, bronchoscopes must be stored in a hanging position, with sufficient space between instruments to avoid cross contamination. (Grade D)

- Valves must not be attached to bronchoscopes during storage. (Grade D)

- Bronchoscopes must be cleaned and disinfected before and after placing in carrying cases as these cases cannot be disinfected. Bronchoscopes should not be stored in carrying cases. (Grade D)

- A record must be kept of each bronchoscope and reusable accessory used on each individual patient. Tracking each step of the decontamination cycle and personnel involved should also be recorded. This will facilitate tracing if an increase in contamination by organisms is identified amongst bronchoscopy patients. (Grade D)

- AERs should be self-disinfected at the beginning of each day. (Grade D)

- AERs must be validated on instillation and following introduction of new disinfectants according to Health Technical Memorandum 01 (HTM-01). (Grade D)

- Sterile water or filtered water should be used for the final rinse. Tap water is not recommended. (Grade D)

- Regular testing of AERs and final rinse water for mycobacteria must be carried out according to HTM-01. (Grade D)

- Compatibility of bronchoscopes with disinfectant and AER manufacturers' instruction should be checked. $(\sqrt{ })$

- A record of which bronchoscope and other reusable equipment are used on an individual patient should be kept and also of the decontamination procedure. $(\sqrt{ })$

- There is currently no known decontamination method that prevents transmission of variant Creutzfeldt-Jakob disease (vCJD). Record keeping and identification of high-risk cases are advised. $(\sqrt{ })$

\section{STAFFING}

- Open troughs of disinfectant are not recommended. (Grade D)

- Staff handling disinfectants should always wear full personal protective equipment in line with $\mathrm{COSHH}$ (control of substances hazardous to health) risk assessment. (Grade D)
- Medical histories of staff should be recorded including preexisting asthma, skin and mucosal sensitivities. (Grade D)

- Pre-employment baseline lung function, such as spirometry, should be measured and recorded. (Grade D)

- Annual lung function measurements, such as spirometry, should be performed on all personnel directly exposed to disinfectants. (Grade D)

- Immunisation against hepatitis B and TB should be confirmed in all bronchoscopy personnel before employment. Vaccinations should be offered if necessary. (Grade D)

- Hypodermic needles or other sharp instruments should not be used to remove tissue samples from biopsy forceps. Blunt-ended needles or sterile plastic toothpicks are preferable. (Grade D)

- Reusable spiked forceps are not recommended. (Grade D)

- A minimum of two qualified nurses are required during bronchoscopy procedures: one assistant nurse and another dedicated to monitoring the patient's response to the medication and procedure. (Grade D)

- A qualified nurse is required to recover a patient after bronchoscopy. (Grade D)

- Advanced procedures may require additional staff. (Grade D)

- In patients with suspected TB, bronchoscopy should be performed in an appropriately engineered and ventilated area, and the bronchoscopy team should use adequate protection, including masks. $(\sqrt{ })$

\section{PATIENT SATISFACTION}

- Verbal and written patient information explaining indications and what to expect during the procedure, and potential complications should be provided to improve patient tolerance. (Grade C)

- Patients should be offered sedation during FB to improve patient tolerance. (Grade B)

- It is sufficient for patients to have no food by mouth for $4 \mathrm{~h}$ and to allow clear fluids by mouth up to $2 \mathrm{~h}$ before bronchoscopy. (Grade D)

- Patients who had sedation should be advised not to drive, sign legally binding documents or operate machinery for $24 \mathrm{~h}$ after the procedure. $(\sqrt{ })$

\section{CONSENT}

- Practitioners undertaking FB should be familiar with, and adhere to the national and local guidance for obtaining informed consent. $(\sqrt{ })$

\section{INTRODUCTION}

\section{Clinical context and need for a guideline}

Flexible bronchoscopy (FB) is a safe and frequently performed procedure for the assessment, diagnosis, and treatment of patients with respiratory disease. The procedure and applications of FB have progressively evolved and expanded since it was first introduced in $1968 .^{1} \mathrm{FB}$ is now established as an essential diagnostic and therapeutic tool in respiratory medicine.

The British Thoracic Society (BTS) published the 2001 guidelines on diagnostic FB. ${ }^{2}$ This document is well respected, used and referenced in the UK and beyond. Literature searches for the 2001 guideline were completed in 1999, but numerous studies have been published in this field since, providing adequate information to revise and update the evidenced-based recommendations. 
In August 2007 the Standards of Care Committee (SOCC) of the BTS invited the interventional pulmonology specialist advisory group of the BTS to produce evidence-based guidelines for advanced diagnostic and therapeutic FB and to update and revise the 2001 BTS guideline on diagnostic FB. ${ }^{2}$ The working party decided to start with the new guideline on advanced diagnostic and therapeutic FB which was published in November 2011. ${ }^{3}$ The proposal to update and revise the BTS guideline on diagnostic FB was approved by the BTS SOCC in November 2010 and work on the guideline started in February 2011. Appendix 1 of this guideline lists the members of the BTS Bronchoscopy Guideline Group.

\section{Target audience of the guideline}

This guideline is aimed primarily at respiratory practitioners in the UK but may be of relevance to other healthcare systems around the world. It is intended to inform those who undertake or intend to undertake $\mathrm{FB}$ and procedures described within the guideline, and to inform other healthcare professionals as to what may be the indications, procedures, likely response and complications of FB in adults. Practitioners using this guideline need to ensure that they follow safe practices and keep patient safety paramount at all times.

\section{Scope of the guideline}

This guideline was formulated following consultation with stakeholders from the medical and nursing professions, patient groups and healthcare management. Basic diagnostic procedures in adults using a flexible bronchoscope are included in the guideline.

\section{Topics covered in the guideline}

- Monitoring of a patient during the procedure.

- Specific precautions, contraindications and complications.

- Sedation, premedication and topical anaesthesia.

- FB in specific patient groups.

- Role of bronchoscopy in infections.

- FB in the ICU.

- Cleaning and disinfection of equipment.

- Staffing and staff safety.

- Diagnostic accuracy and specific procedures.

- Patient satisfaction and patient care.

Topics not covered in the guideline

- Training in bronchoscopy (The BTS is producing separate guidance on training).

- Advanced diagnostic and therapeutic FB. ${ }^{3}$

- Rigid bronchoscopy.

- FB used for intubation, percutaneous tracheostomy placements and intraoperative complications.

- Paediatric FB.

- FB performed under general anaesthetic.

\section{METHODOLOGY}

This guideline is based on the best available evidence and is a revised update of the BTS guideline on diagnostic $\mathrm{FB}^{2}$ published in 2001. The methodology used to write the guideline adheres strictly to the criteria as set by the BTS guideline production manual and the Appraisal of Guidelines for Research and Evaluation (AGREE) collaboration in the document 'The AGREE Instrument', which is available online: http://www. agreecollaboration.org/1/agreeguide/

\section{Clinical questions and literature search}

Clinical questions were gathered in the PICOT (Patient, Intervention, Control, Outcome and Time) format to define the scope of the guideline and inform the literature search.

Systematic electronic database searches were conducted to identify potentially relevant studies for inclusion in the guideline. For each topic area the following databases were searched: Ovid MEDLINE (from 1988) (including MEDLINE In Process), Ovid EMBASE (from 1988), Ovid CINAHL (from 1982) and the Cochrane Library (from 1992) (including the Cochrane Database of Systematic Reviews, the Database of Abstracts of Reviews of Effects, the Cochrane Central Register of Controlled Trials, the Health Technology Assessment database and the NHS Economic Evaluation Database). The search strategies are available in appendix 2 .

The searches were first run in January 2011 and were updated in January 2012 and June 2012. Searches were saved and alerts sent via email on a monthly basis to identify newly published literature to date. Searches included a combination of indexed terms and free text terms, and were limited to English language publications only. The initial search identified 22865 potential papers.

\section{Appraisal of the literature}

Appraisal was performed using the criteria stipulated by the AGREE collaboration. One individual (IDR) read the title and abstract of each article retrieved by the literature searches and decided whether the paper was (1) definitely relevant, (2) possibly relevant or (3) not relevant to the project. A total of 9121 papers were identified to review for inclusion of the guideline. Criteria formulated for initial screening of the abstracts into these three groups were:

- Whether the study addressed the clinical question.

- Whether the appropriate study type was used to produce the best evidence to answer the clinical question.

- Abstract was in English.

- Studies in which exclusively rigid bronchoscopy was used were not evaluated.

- Abstracts were not rejected on the basis of the journal of publication, country in which the research was performed or published or the date of publication.

The full paper was obtained for all relevant or possibly relevant abstracts and allocated to the relevant section(s):

- Sedation, premedication and topical anaesthesia.

- Monitoring, precautions, contraindications and complications.

- Specific conditions.

- Bronchoscopy in the ICU.

- Infections.

- Cleaning, disinfecting and staff safety.

- Diagnostic accuracy.

- Patient satisfaction and consent.

The first screening process identified 9121 abstracts to be reviewed, 1824 abstracts did not meet the criteria as set out above, 1504 studies used FB to collect samples for research purposes and 1731 case reports in FB were identified. Two guideline reviewers independently reviewed the abstracts of the remaining 4062 studies to identify 2197 papers to be appraised for the guideline. The two leads for each section independently appraised each paper assigned to them using the Scottish Intercollegiate Guidelines Network (SIGN) critical appraisal checklists. A web-based guideline development tool (http://www. bronchoscopy-guideline.org, designed by IDR) was used for 1505 critical appraisals of 522 studies. The website enabled 
each pair of reviewers to collaborate online and produce evidence tables electronically. The reliability of the evidence in each individual study was graded using the SIGN critical appraisal checklists and is shown in the evidence tables $(++,+$ or -). The body of evidence for each recommendation was summarised into evidence statements and graded using the SIGN grading system (see table 1). Disagreements were resolved by discussion with the section partner and the Guideline Group.

\section{Considered judgement and grading of the evidence}

The Guideline Group used the online derived evidence tables to judge the body of evidence and grade recommendations for this guideline. The evidence tables are available in appendix 3 for review and are published electronically on the BTS website.

When evidence was lacking to answer the formulated clinical questions, expert opinions were obtained for formal consensus statements using the Delphi method.

The following were considered in grading the recommendations:

- The available volume of the body of evidence.

- How applicable the obtained evidence was in making recommendations for the defined target audience of this guideline.

- Whether the evidence was generalisable to the target population for the guideline.

- Whether there was a clear consistency in the evidence obtained to support recommendations.

- What the implications of recommendations will be on clinical practice in terms of recourses and skilled expertise.

- Cost effectiveness was not reviewed in detail as in-depth economic analysis of recommendations falls beyond the scope of this guideline.

Recommendations were graded from A to D according to the strength of the evidence, as listed in table 2. Important practical points lacking any research evidence were highlighted as 'good practice points' $(\sqrt{ })$.

The grading system used to grade recommendations for this revised guideline differs from the system used to grade the recommendations for the 2001 BTS guideline on diagnostic FB, shown in table 3 . Readers of the guideline are therefore advised to review both grading systems and to note that apparent changes in recommendations between guidelines may be due to

Table 1 Revised grading system for levels of evidence in evidence-based guidelines

\begin{tabular}{ll}
\hline Grade & Evidence \\
\hline $1++$ & $\begin{array}{l}\text { High-quality meta-analyses, systematic reviews of RCTs or RCTs with a } \\
\text { very low risk of bias }\end{array}$ \\
$1+$ & $\begin{array}{l}\text { Well conducted meta-analyses, systematic reviews of RCTs or RCTs with } \\
\text { a low risk of bias }\end{array}$ \\
Meta-analyses, systematic reviews or RCTs, or RCTs with a high risk of \\
bias
\end{tabular}

Table 2 Grades of recommendations used for this guideline

Grade Type of evidence

A At least one meta-analysis, systematic review or RCT rated as 1++ and directly applicable to the target population or

A systematic review of RCTs or a body of evidence consisting principally of studies rated as $1+$ directly applicable to the target population and demonstrating overall consistency of results

B A body of evidence including studies rated as 2++ directly applicable to the target population and demonstrating overall consistency of results or Extrapolated evidence from studies rated as $1++$ or $1+$

C A body of evidence including studies rated as $2+$ directly applicable to the target population and demonstrating overall consistency of results or Extrapolated evidence from studies rated as $2++$

D Evidence level 3 or 4 or Extrapolated evidence from studies rated as $2+$

$\sqrt{ } \quad$ An important practical point for which there is no research evidence, no is there likely to be any research evidence. The guideline group wishes to emphasise these as good practice points

$\mathrm{RCT}$, randomised controlled trial.

the use of different grading systems rather than a change in the recommendation itself.

\section{Drafting of the guideline}

The Guideline Group corresponded regularly by email and meetings of the full group were held in February 2011, September 2011, December 2011, March 2012, May 2012 and June 2012. The guideline was discussed at an open session at the BTS Summer Meeting in July 2012. A revised draft guideline document was circulated to all the relevant stakeholders for consultation in July 2012 followed by a period of online consultation. The BTS SOCC reviewed the draft guideline in June 2012. A list of stakeholders is available for review in appendix 4 of this guideline.

Table 3 Grading system used in the 2001 British Thoracic Society (BTS) guideline on diagnostic flexible bronchoscopy

\begin{tabular}{ll}
\hline Level & Evidence \\
\hline la & Evidence obtained from meta-analysis of RCTs \\
Ib & Evidence obtained from at least one RCT \\
Ila & $\begin{array}{l}\text { Evidence obtained from at least one well designed controlled study } \\
\text { without randomisation }\end{array}$ \\
IIb & $\begin{array}{l}\text { Evidence obtained from at least one other type of well designed } \\
\text { quasi-experimental study }\end{array}$ \\
III & $\begin{array}{l}\text { Evidence obtained from well designed non-experimental descriptive } \\
\text { studies such as comparative studies, correlation studies and case- } \\
\end{array}$ \\
IV & $\begin{array}{l}\text { Evidence obtained from expert committee reports of opinions and/ } \\
\text { or clinical experiences of respected authorities }\end{array}$
\end{tabular}

\section{Grade Type of recommendation}

A (la, lb) Requires at least one RCT as part of a body of literature of overall good quality and consistency addressing the specific recommendation

B (Ila, Ilb, Requires availability of well conducted clinical studies but no RCTs III) on the topic of recommendation

C (IV) Requires evidence from expert committee reports or opinions and/ or clinical experience of respected authorities. Indicates absence of directly applicable studies of good quality

$\mathrm{RCT}$, randomised controlled trial. 
The Guideline Group members adhered to the BTS policy for the Declaration of Interests, and if appropriate, specific interests are declared in appendix 1.

The guideline will be reviewed within 5 years from the date of publication (2018).

\section{AUDIT AND RESEARCH RECOMMENDATIONS}

Audit:

- All those undertaking FB are advised to maintain personal records of each procedure, including indication, outcome and complications for audit purposes.

- Periodic audit of bronchoscopy practice, including patient satisfaction surveys.

Research:

- Utility of all bronchoscopic samples, including brush and BAL for phenotyping and genotyping in patients with advanced non-small cell lung cancer (NSCLC).

- Randomised assessment of the utility of bronchoscopy in the relief of lobar collapse/atelectasis in ventilated patients.

- Further assessment of the use of jet ventilation during bronchoscopy in mechanically ventilated patients.

- Further assessment of optimal analgesia and sedation for safe bronchoscopy in mechanically ventilated patients.

\section{Training}

Training in FB does not fall in the scope of this guideline.

\section{Audit standards}

The following standards provide criteria which may form the basis of future audits:

- A serious adverse event rate of $<1 \%$ (box 1 ).

- A 'safe sedation policy' and appropriate training in sedation for all bronchoscopy unit staff, including trainees and interval audit of sedation practice.

- Utilisation of the bronchoscopy safety checklist (see appendix $5)$.

- An $85 \%$ diagnostic rate for FB with visible endobronchial tumour.

- Periodic patient feedback to inform improvement and revision of the endoscopy service.

\section{MONITORING, PRECAUTIONS AND COMPLICATIONS}

FB is an increasingly important diagnostic, well tolerated procedure that can be performed safely on an outpatient basis. In the largest retrospective series $(n=20986)$, serious complications occurred in $1.1 \%$ with a mortality of $0.02 \% .^{4}$ The commonest adverse events reported, though not universally in all

\section{Box 1 Suggested serious adverse events}

- Severe bleeding (see table 5)

- Cardiac arrhythmia requiring treatment

- Seizures

- Myocardial infarction/pulmonary oedema

- Pneumothorax requiring aspiration/intercostal drain

- Oversedation requiring ventilatory support or reversal

- Hospitalisation

- Admission to intensive care unit

- Death studies, included tachycardia/bradycardia, major and minor bleeding, bronchospasm/laryngospasm, cough, dyspnoea, sore throat, apnoea, seizure, desaturation, pneumothorax and pulmonary oedema. Other smaller studies report complication rates of $5-32 \%$, and mortality rates of $0-0.8 \%$, but these studies are limited by their retrospective nature, the variable definition of adverse events and limited follow up.

Smaller prospective studies suggest that the rate of adverse events may be higher than previously reported. Hehn et $a l^{5}$ demonstrated respiratory complications in 4.3\%, nonrespiratory complications in $2.8 \%$ and mortality in $0.1 \%$. In addition, Bechara et $a l^{6}$ reported adverse events in $35 \%$ of 300 bronchoscopies performed, $60 \%$ of which were classified as mild and $8 \%$ as severe. Approximately $6 \%$ of patients were hospitalised and procedure-related deaths occurred in $1.4 \% .^{6}$

There is an increased risk of adverse events with increasing age but the absolute frequency is low. Chronological age should not be a contraindication for bronchoscopy. ${ }^{5} 7$ Patient position during the procedure does not influence complication rates, with the exception that desaturation $>4 \%$ is more common in the sitting position. ${ }^{8}$

Many factors will influence the risk of complications, including patient characteristics and factors related to the bronchoscopic unit (including sedation practice and the sampling procedures employed). Utilisation of a WHO safety checklist aids in identifying specific possible complications (see appendix 5) Complications, particularly serious adverse events (box 1), patient satisfaction and efficacy should be routinely monitored by every bronchoscopy unit.

Recommendation

- All patients undergoing bronchoscopy should have heart rate, blood pressure and oxygen saturation recorded repeatedly, including before, during and after the procedure. (Grade D)

Good practice points

- All bronchoscopy units should undertake periodic audit of bronchoscopic performance, including efficacy, complications and patient satisfaction surveys. $(\sqrt{ })$

- All Trusts should have a 'safe sedation policy', and ensure all bronchoscopy unit staff, including trainees, receive appropriate training. $(\sqrt{ })$

\section{Hypoxaemia}

Monitoring patients with pulse oximetry during bronchoscopy is an accurate non-invasive method for assessing hypoxaemia. ${ }^{9-11}$ Significant decreases in oxygen saturation are commonly seen during bronchoscopy, commencing with administration of sedation and worsening on passage through the vocal cords. ${ }^{8} 9$ 12-16 Patient positioning 81016 and intra-procedural sampling may also influence oxygen saturations as may airway suctioning. ${ }^{17} 18$ Van Zwam et al ${ }^{8}$ described a twofold greater incidence of desaturation $>4 \%$ and $\mathrm{SpO}_{2}<90 \%$ in the sitting position compared with supine. The use of preprocedure oxygen and the specific sampling procedure (BAL vs wash vs brush vs biopsy) was predictive of a higher rate of desaturation episodes $(<90 \%)$, but baseline saturations were not (desaturation to $<90 \%$ was seen in: BAL $89 \%$, wash $44 \%$, brush $15 \%$, biopsy 10\%). ${ }^{19}$ Milman et $\mathrm{l}^{11}$ (using benzodiazepine premedication without oxygen supplementation) demonstrated that $38 \%$ of patients desaturate $\left(\mathrm{SpO}_{2}<90 \%\right)$ before bronchoscopy, increasing to $80 \%$ of patients during the procedure. The proportion remains high after the procedure, with $60 \%$ of patients desaturating. Similarly, more severe desaturation $\left(\mathrm{SpO}_{2}<85 \%\right)$ was seen in $10 \%$, $35 \%$ and $15 \%$, respectively. No differences in oxygen saturation 
were described in a comparison of trans-nasal or trans-oral bronchoscopic approaches. ${ }^{20}$

The majority of desaturations are transient and do not require specific intervention. ${ }^{21}$ Supplemental oxygen given via nasal or pharyngeal catheter can reduce the incidence, degree or duration of desaturation. ${ }^{11} 1622$ In patients undergoing BAL and TBLBs for diffuse ILD, significant hypoxaemia during bronchoscopy can be avoided with routine supplemental oxygen compared with breathing room air alone, but the proportion of patients requiring supplemental oxygen in general bronchoscopic practice is variable, ranging between $5 \%$ and $32 \%$, and is dependent on forced expiratory volume in $1 \mathrm{~s}\left(\mathrm{FEV}_{1}\right)$ (or peak expiratory flow rate (PEFR)). ${ }^{12} 21$

Patients with abnormal PEFR demonstrate an increased requirement for oxygen supplementation (PEFR $<60 \%$ predicted: $58 \%$, $<45 \%$ predicted: $83 \%)^{12}$ and Jones and O'Driscoll ${ }^{21}$ demonstrated a greater risk of desaturation to $<90 \%$ and need for oxygen supplementation with declining $\mathrm{FEV}_{1}$ (table 4).

In studies examining the effect of BAL volume on oxygen saturation, inconsistent results have been reported. ${ }^{15} 23$

There are no studies that address a safe saturation threshold. Schiffman et $a l^{22}$ noted that significant desaturation could be prevented with $4 \mathrm{~L} / \mathrm{min}$ oxygen supplementation but this did not impact on the rate of cardiac arrhythmia (sinus tachycardia, sinus bradycardia, ventricular or atrial premature contractions). Similarly, Lundgren et al ${ }^{18}$ did not demonstrate an increase in cardiac arrhythmia, despite hypoxaemia and increased cardiac work in $30 \%$ of patients. Two further studies demonstrated no significant increase in the frequency of bradycardia/tachycardia or premature atrial/ventricular activity with or without oxygen supplementation. ${ }^{11} 20$

Hypoxaemia can be effectively minimised by using a nasal or pharyngeal catheter for oxygen supplementation at either 2 or $3 \mathrm{~L} / \mathrm{min} .{ }^{11}$ In hypoxaemic respiratory failure $(\mathrm{RR}>35$, arterial oxygen pressure $\left(\mathrm{PaO}_{2}\right) /$ fractional inspired oxygen $\left.\left(\mathrm{FiO}_{2}\right)<200\right)$ non-invasive positive pressure ventilation $\left(\mathrm{FiO}_{2} 0.5\right.$, adjusted to maintain $\mathrm{SaO}_{2}>92 \%$ ) is superior to a high-flow venturi mask (constant $\mathrm{FiO}_{2}$ 0.9) during bronchoscopy for nosocomial pneumonia. ${ }^{24}$ Several studies, including those post BAL, suggest that hypoxaemia may persist for at least $2 \mathrm{~h}$ after the procedure but there is no evidence relating to the duration for oxygen supplementation post procedure. ${ }^{25-27}$

Oxygen supplementation should be used in patients with persistent significant desaturation $\left(\mathrm{SaO}_{2}\right.$ change $>4 \%$ or $\mathrm{SaO}_{2}<90 \%$ ). Target oxygen saturations should be consistent with the principles of published guidance on oxygen therapy.

Evidence statements

- Hypoxaemia is common during bronchoscopy, it is frequently transient, and only considered significant if

Table 4 Patients showing a fall in $\mathrm{SpO}_{2}$ and requiring supplemental oxygen during FB

\begin{tabular}{lll}
\hline $\mathrm{FEV}_{\mathbf{1}}$ in litres & $\begin{array}{l}\text { Patients with } \\
\mathrm{SpO}_{2}<90 \%(\%)\end{array}$ & $\begin{array}{l}\text { Patients requiring } \\
\text { supplemental oxygen (\%) }\end{array}$ \\
\hline$<0.5$ & 93 & 71 \\
$0.51-1.0$ & 48 & 32 \\
$1.01-1.5$ & 25 & 14 \\
$1.51-2.0$ & 17 & 8 \\
$>2.0$ & 10 & 5 \\
\hline
\end{tabular}

Adapted from Jones and O'Driscoll ${ }^{21}$

$\mathrm{FEV}_{1}$, forced expiratory volume in $1 \mathrm{~s} ; \mathrm{SpO}_{2}$, pulse oximeter oxygen saturation. prolonged ( $>1 \mathrm{~min})$. Hypoxaemia is more common in the sitting position, with oral or intravenous sedation, with decreasing $\mathrm{FEV}_{1}$ or PEFR, or in patients who require supplemental oxygen before the procedure. The use of suction can exacerbate hypoxaemia. (Evidence level $2++$ )

- Pulse oximetry demonstrates excellent correlation with arterial gas analysis. (Evidence level 3)

- The use of supplemental oxygen can reduce the severity of hypoxaemia, either by nasal or pharyngeal catheter, at flow rates of at least $2 \mathrm{~L} / \mathrm{min}$. (Evidence level $2+$ ).

- Oxygen supplementation should be targeted to patients with persistent significant desaturation $\left(\mathrm{SpO}_{2}\right.$ change $>4 \%$ or $\mathrm{SpO}_{2}<90 \%$ ). Target oxygen saturations should be consistent with the principles of published guidance on oxygen therapy. (Evidence level 3)

\section{Recommendations}

- Patients should be monitored by continuous pulse oximetry during bronchoscopy. (Grade C)

- Oxygen supplementation should be used when desaturation is significant $\left(\mathrm{SpO}_{2}>4 \%\right.$ change, or $\left.\mathrm{SpO}_{2}<90 \%\right)$ and prolonged $(>1 \mathrm{~min})$ to reduce the risk of hypoxaemia-related complications. (Grade D)

- The risks of hypoxaemia-related complications are associated with baseline $\mathrm{SaO}_{2}$ and lung function, comorbidity, sedation and procedural sampling. Fitness for bronchoscopy should incorporate an assessment of these elements, and appropriate monitoring and preprocedure optimisation. (Grade D)

\section{Cardiac complications}

Hypoxaemia related to $\mathrm{FB}$ is commonly associated with an increase in cardiac workload with elevations of heart rate (approximately 40\% above baseline), blood pressure (a rise of $30 \%$ above baseline) and cardiac index (approximately 17-32\% of baseline). ${ }^{14} 182829$ Despite this, major arrhythmias are rare during bronchoscopy but believed to be related to myocardial ischaemia. The rate-pressure product (heart rate $\times$ systolic blood pressure) during bronchoscopy can approach or exceed the level associated with silent myocardial ischaemia, particularly in patients with hypertension. ${ }^{14} 1828$ Increases in systolic blood pressure and heart rate during the bronchoscopy are associated with ECG change in 15\% (ST-T change in 4\%, transient right bundle branch block in 3\%). ${ }^{29}$ In addition, ECG changes correlate with older age, higher pack-year smoking history but not lung function or changes in oxygen saturation. Cardiac strain has been reported to occur in $21 \%$ of patients over the age of 60 years. $^{29}$

\section{Arrhythmia}

Schiffman et $a^{22}$ demonstrated that bronchoscopy was associated with sinus tachycardia in $55-58 \%$, sinus bradycardia in $5-8 \%$, premature ventricular contraction in $8 \%$ and atrial premature contraction in $3-5 \%$, with no significant difference according to oxygen supplementation. Payne et $a l^{20}$ demonstrated a $60 \%$ prevalence of baseline minor arrhythmia and 5\% incidence of new minor arrhythmia with bronchoscopy.

Katz et $a l^{30}$ documented $12 \%$ of patients with at least an occasional atrial or ventricular ectopic beat before the procedure compared with $80 \%$ of patients during or after bronchoscopy. Major cardiac arrhythmias (defined as five or more atrial ectopics/min, supraventricular tachycardia, or five or more ventricular ectopics/min, multiform ectopic beats, couplets or ventricular tachycardia) increased from $4 \%$ before the procedure to $40 \%$ during/after the procedure. Atrial arrhythmias occur at widely differing stages of the procedure, but ventricular 
arrhythmias occur mainly on passage through the vocal cords. Maximum ventricular arrhythmia is correlated with minimum oxygen saturations. Asymptomatic ST-T wave changes occur in $6 \%$ of patients, typically correlating with maximum heart rates. Oxygen saturations can remain lower than preprocedure levels $3 \mathrm{~h}$ after bronchoscopy in $30 \%$ of patients.

\section{Myocardial infarction}

Acute MI is considered a contraindication to bronchoscopy within 4-6 weeks. Dweik et $a l^{31}$ retrospectively analysed the safety of bronchoscopies within 30 days of an acute MI and noted that mortality $(5 \%)$ was limited to patients with active ischaemia at the time of bronchoscopy.

Evidence statements

- Bronchoscopy increases cardiac rate, blood pressure and cardiac index. The rate pressure product is often sufficient to cause myocardial ischaemia. (Evidence level 2+)

- Sinus tachycardia and atrial or ventricular premature contractions are the commonest arrhythmia noted before, during and after bronchoscopy. (Evidence level 3)

- Ventricular arrhythmia (mostly premature contraction, bi and trigeminy) occurs most commonly on passage through the cords and is associated with low oxygen saturations. Oxygen saturations may remain below preprocedure levels for over $3 \mathrm{~h}$ in a third of patients. (Evidence level 3)

- Myocardial ischaemia during bronchoscopy is related to heart rate and blood pressure, rather than oxygen saturation per se. It also correlates with increasing age and smoking history. (Evidence level 3)

- Bronchoscopy within 30-days of acute MI is associated with a 5\% mortality (related to active ischaemia). In the absence of active ischaemia, when good clinical justification is made, bronchoscopy can be performed. (Evidence level 3)

- Incidence of cardiac arrhythmia is not affected by oxygen supplementation. (Evidence level 3)

Recommendations

- Continuous ECG monitoring should be used when there is a high clinical risk of arrhythmia. (Grade D)

- When there is a high risk of arrhythmia, oxygen saturations, pulse rate and blood pressure should be optimised. Appropriate aftercare monitoring and instructions should be given. (Grade D)

Good practice points

- Resuscitation equipment should be readily available. $(\sqrt{ })$

- Intravenous access should be established before sedation is given and maintained until discharge. $(\sqrt{ })$

\section{Bleeding complications}

It is difficult to subclassify bleeding during FB into minor, moderate or severe based on an estimate or measures of blood loss during the procedure. Aspirated blood is collected and mixed with saline, adrenaline and suctioned secretions and an accurate measure is not possible. Classification by the type of clinical intervention necessary to stop the bleeding and stabilise the patient is an easier and reproducible measure of bleeding (see table 5, adapted from Ernst et $a l^{32}$ ).

Minor bleeding occurs in $0.19 \%$ and severe bleeding in $0.26 \%$ of bronchoscopies. ${ }^{4}$ Clinical risk factors for bleeding correlate with abnormal coagulation but the rate of biopsy-related bleeding in patients with clinical risk factors is only $11 \%$ (box 2). In patients with known abnormal coagulation, bleeding occurred in a similar $11 \% .^{33}$ The type of biopsy, abnormal coagulation, platelets, haemoglobin or creatinine does not reliably or consistently predict bleeding risk for bronchoscopy.

\begin{tabular}{ll} 
Table 5 & Classification of bleeding during bronchoscopy \\
\hline No bleeding & $\begin{array}{l}\text { Traces of blood with no need for continuous suctioning } \\
\text { Bleeding stops spontaneously }\end{array}$ \\
Mild bleeding & $\begin{array}{l}\text { Continued suctioning of blood from the airways } \\
\text { Bleeding stops spontaneously } \\
\text { Intubation of the biopsied segment with the bronchoscope } \\
\text { into the wedge position }\end{array}$ \\
Moderate & $\begin{array}{l}\text { Use of adrenaline or cold saline to stop bleeding } \\
\text { bleeding }\end{array}$ \\
Severe bleeding & $\begin{array}{l}\text { Placement of bronchus blocker or catheter, applying fibrin } \\
\text { sealant } \\
\text { Resuscitation, blood transfusion, admission to critical care unit } \\
\text { or death }\end{array}$ \\
\hline Adapted from Ernst et al. ${ }^{32}$
\end{tabular}

Over two-thirds of patients who develop bleeding have normal coagulation and no clinical risk factors for bleeding.

The majority of bleeding is mild to moderate with only $3 \%$ estimated at being more than $100 \mathrm{~mL}$. Approximately $90 \%$ of bleeding stops spontaneously or requires local vasoconstrictor therapy only (adrenaline/cocaine). ${ }^{33}$ Appendix 6 provides a management approach to bleeding complications during FB.

At bronchoscopy, clinically significant bleeding was seen in $0.83 \%$, increasing to $1.9 \%$ with biopsy, including TBLB. ${ }^{34}$ TBLB caused mild-moderate bleeding in $0.8 \%$ whereas EBB bleeding occurs in only $0.45 \% .^{34}$ Severe bleeding is more common in TBLB than EBB but remains $<20 \mathrm{~mL}$ in the majority of cases of TBLB (92\%). ${ }^{35}$ Spontaneous resolution of bleeding occurred in two-thirds and was treated with local instillation of adrenaline in the remainder. ${ }^{35}{ }^{34}$ Platelet levels or coagulation studies prior to TBLB do not predict bleeding in procedures in which bleeding occurs. ${ }^{35}$

Diette et $a l^{36}$ prospectively studied 720 procedures, including 38 lung transplants. Transplant recipients are more likely to have TBLB and to receive aspirin therapy, to have blood loss $>25 \mathrm{~mL}$ and to have the procedure terminated early for bleeding. In multivariate analysis, independent predictors of greater blood loss included lung transplant, performance of TBLB, longer procedure time and older patient age. A smaller prospective study found that bleeding was quantitatively similar between patients with and without lung transplant and typically minor. ${ }^{35}$

Weiss et $a l^{37}$ prospectively reported 66 bronchoscopies in 47 bone marrow transplant recipients with thrombocytopenia (20\% had platelets $<20000 / \mathrm{mL} ; 67 \%$ platelets $<50000 / \mathrm{mL}$; $88 \%$ platelets $<100000 / \mathrm{mL})$. Bleeding-related complications were reported in $6.9 \%$ and were usually minor.

In renal failure, including haemodialysis and non-dialysis patients, bronchoscopic biopsy and transbronchial needle aspiration (TBNA) were associated with an overall complication rate of $8 \% .^{38}$

The concomitant use of clopidogrel with transbronchial biopsy leads to excessive $(>100 \mathrm{~mL})$ bleeding in patients taking

\section{Box 2 Clinical risk factors for abnormal coagulation}

- Anticoagulant therapy

- Evidence of liver disease

- History, family history or physical evidence of bleeding tendency

- Active bleeding or pre-procedure transfusion 
clopidogrel alone (89\% vs 3.4\%) and clopidogrel with aspirin $(100 \%$ vs $3.4 \%) .{ }^{32}$ Bleeding rates are significantly higher in all categories of bleeding (minor/moderate/severe) but can be controlled in most instances by bronchoscopic means. Need for transfusion or death following haemorrhage secondary to clopidogrel is rare. ${ }^{32}$ See appendix 7 for an algorithm for the management of patients on warfarin or clopidogrel undergoing FB.

Evidence statements

- Minor bleeding occurs in $0.19 \%$ and severe bleeding in $0.26 \%$ of bronchoscopies. (Evidence level 3)

- The routine performance of coagulation studies, platelet or haemoglobin counts are of no value in predicting the risk or severity of bleeding. (Evidence level 3)

- Coagulation studies, platelet count and haemoglobin values should be estimated when clinical risk factors indicate a likelihood of abnormal coagulation. However, over two-thirds of patients with significant bleeding possess normal coagulation and no clinical risk factors for bleeding. (Evidence level 3)

- Bleeding complications in patients with thrombocytopenia undergoing bronchoscopy and lavage are approximately $7 \%$. No data are available regarding the safety of TBLB or EBB in thrombocytopenia but the majority of bleeding complications relate to epistaxis. (Evidence level 3)

- Clopidogrel causes bleeding, ranging from mild to severe, when performing TBLB. (Evidence level $2+$ )

- TBLB causes a twofold increase in the risk of mild-moderate bleeding and a threefold increase in the risk of severe bleeding compared with EBB. However, the overall risk remains small and TBLB rarely causes significant blood loss (92\% of patients experience blood loss $<20 \mathrm{~mL}$ ) and typically resolves spontaneously or with endoscopic instillation of cocaine/adrenaline. (Evidence level 3)

- Bronchoscopic biopsy and TBNA in patients receiving haemodialysis, or in patients with renal failure without dialysis, result in a higher rate of bleeding complications ( 8\%; 4\% major, $4 \%$ minor) than the general population. (Evidence level 3 )

- Lung transplantation may predispose patients to greater blood loss at bronchoscopic biopsy, including TBLB. (Evidence level 2-)

\section{Recommendations}

- Perform coagulation studies, platelet count and haemoglobin concentration when there are clinical risk factors for abnormal coagulation. (Grade D)

- Bronchoscopy with lavage can be performed with platelet counts $>20000$ per $\mu \mathrm{L}$. Liaise with the local haematology team regarding the need for platelet transfusion before bronchoscopy if EBB or TBLB is planned. (Grade D)

- Discontinue clopidogrel 7 days prior to consideration of EBB and TBLB. Low-dose aspirin alone can be continued. (Grade C)

\section{Good practice points}

- Anticoagulants should be managed according to published guidelines as set out in appendix 7 of this guideline. $(\sqrt{ })$

- The risk of biopsy needs to be weighed against the potential for benefit and appropriate informed consent obtained. $(\sqrt{ })$

\section{Pneumothorax}

Pneumothorax following bronchoscopy for any indication occurs at a rate of 1 in $1000(0.1-0.16 \%)^{4}{ }^{39}$ but was as high as $0.4-0.8 \%$ in some smaller series. ${ }^{40}{ }^{41}$ In contrast the rate of pneumothorax in TBLB has been reported to be significantly higher between $1 \%$ and $6 \%{ }^{43942} 43$ or higher still in TBLB of diffuse abnormality (9\%). ${ }^{43-46}$ TBLB remains a safe outpatient procedure with a low incidence of delayed complications. ${ }^{47}$

In relation to all adverse events, pneumothorax represents approximately $10 \%$ of all complications but rarely complicates bronchoscopy without TBLB or therapeutic bronchoscopy. ${ }^{4}$ Pneumothorax is rarely total and often delayed ( $40 \%$ of pneumothoraces) ${ }^{4}$ and when present may require intercostal tube drainage (40-70\% of cases). ${ }^{39} 42 \quad 43 \quad 47$ The frequency of pneumothorax is related to age and the number of TBLBs. ${ }^{5} 44$ Routine performance of a chest $\mathrm{x}$-ray after TBLB rarely provides useful clinical information in the absence of symptoms ${ }^{43} 4448$ and may not be required. In the absence of a routine chest x-ray, monitoring for the development of symptoms associated with pneumothorax should continue for $2 \mathrm{~h}$. The rate of pneumothorax following TBLB does not appear significantly different with or without fluoroscopy, ${ }^{45}$ but fluoroscopy may be of value to improve the diagnostic yield in focal rather than diffuse lung disease (focal $4.3 \%$, diffuse $9 \%$ ). ${ }^{43}$

Evidence statements

- Risk of pneumothorax from all bronchoscopic procedures is 1 in $1000(0.1 \%)$ but increases to between 1 in 100 to 1 in 16 (1-6\%) following TBLB. (Evidence level 3)

- Pneumothorax may be delayed (up to $2 \mathrm{~h}$ in $40 \%$ of cases) and may require intercostal tube drainage (Evidence level 3)

- Routine performance of a chest x-ray after TBLB rarely provides useful clinical information in the absence of symptoms. (Evidence level 3)

- Fluoroscopy may reduce the rate of pneumothorax in focal lung disease. (Evidence level 3)

Recommendations

- A chest radiograph should be obtained if a patient is symptomatic or there is a clinical suspicion of possible pneumothorax after TBLB. (Grade D)

- Fluoroscopic screening may improve diagnostic yield of TBLB in focal but not diffuse lung disease. (Grade D)

- Patients should be advised of the potential for delayed complications following TBLB and provided with written information regarding likely symptoms and action required. (Grade D)

\section{Fever and infection}

Post bronchoscopy fever (PBF) is not reported in a large prospective study of complications in over 20000 patients ${ }^{4}$ but appears relatively common in other smaller prospective studies focusing on $\mathrm{PBF}(5-10 \%){ }^{49}{ }^{50} \mathrm{PBF}$ is most typically seen approximately $8 \mathrm{~h}$ (range $4-24 \mathrm{~h}$ ) following BAL (13\%) when it is associated with an acute inflammatory response characterised by fever $>38^{\circ} \mathrm{C}$, neutrophilic leucocytosis, elevated C-reactive protein, fibrinogen and proinflammatory cytokines, ${ }^{50-52}$ and an absence of bacteraemia. ${ }^{495053}$ Fever is typically less than $40^{\circ} \mathrm{C}$, lasts on average $14 \mathrm{~h}$ but is rarely accompanied by a chest $\mathrm{x}$-ray infiltrate. ${ }^{50}$ Antibiotic prophylaxis does not prevent PBF, pneumonia or the proinflammatory cytokine response. ${ }^{53} 54$

A true bacteraemia post bronchoscopy occurs in $6-8 \%$ of patients, ${ }^{49} 55$ most commonly coagulase negative or positive staphylococci, non-haemolytic or $\beta$-haemolytic streptococci, Citrobacter or Klebsiella species. ${ }^{49} 5455$ National Institute for Health and Clinical Excellence guidance in March 2008 also concluded that 'Antibacterial prophylaxis is not recommended for the prevention of endocarditis in patients undergoing procedures of the upper and lower respiratory tract (including bronchoscopy)'. ${ }^{56}$ Evidence statements

- PBF is common, particularly after BAL, and associated with a non-infective acute inflammatory response, typically starting after discharge from hospital. (Evidence level $2++$ ) 
- Antibiotic prophylaxis does not prevent PBF or pneumonia. (Evidence level 1++)

- Bacteraemia occurs in 6-8\% of patients undergoing bronchoscopy. (Evidence level 3)

Recommendations

- Patients should receive written information regarding PBF and appropriate management advice. (Grade C)

- Antibiotic prophylaxis is not warranted before bronchoscopy for the prevention of endocarditis, fever or pneumonia. (Grade B)

\section{SAFETY OF FB IN SPECIFIC MEDICAL CONDITIONS Patients with asthma}

The safety of bronchoscopy in asthma has been studied in research and clinical settings. Bronchoscopy often causes a fall in $\mathrm{FEV}_{1}$. In healthy volunteers the mean fall in $\mathrm{FEV}_{1}$ has been reported on average to be between $9 \%$ and $17 \%,{ }^{15}{ }^{57}$ however it can be over $20 \% .{ }^{58}$ In patients with asthma the mean fall has been reported to be $10-26 \% .^{15} 5759$ The majority of studies comparing the fall in $\mathrm{FEV}_{1}$ between patients with asthma and healthy volunteers found no difference between the groups. ${ }^{15} 5860$ Patients with increased bronchial hyper-reactivity may have a greater fall in $\mathrm{FEV}_{1}{ }^{57}{ }^{61}$ however this has not been universally reported. ${ }^{58}$ In particular, BAL can cause a fall in the $\mathrm{FEV}_{1} .5859$

Complication rates post bronchoscopy range between $3.5 \%$ and $12 \%$ depending on how the complications are reported and what procedures were performed. ${ }^{15}{ }^{60-63}$ In addition, patients with severe asthma are more likely to require oral corticosteroids post bronchoscopy. ${ }^{58}$ Many of the studies use bronchodilators prior to bronchoscopy to increase the $\mathrm{FEV}_{1} .{ }^{57} 58 \quad 6062$ Although this does not seem to reduce the percentage fall in $\mathrm{FEV}_{1}$ it may increase the absolute $\mathrm{FEV}_{1}$ at the end of the procedure, as the patient starts from a higher baseline.

Evidence statements

- Up to $10 \%$ of patients with asthma may develop respiratory symptoms post bronchoscopy. (Evidence level 2-)

- BAL is associated with more symptoms in patients with asthma compared with bronchoscopy alone. (Evidence level 2-)

\section{Recommendation}

- Patients' asthma control should be optimised prior to bronchoscopy, especially when BAL is likely to be performed. (Grade C)

Good practice point

- Nebulised bronchodilators should be considered before bronchoscopy in patients with asthma. $(\sqrt{ })$

\section{Patients with COPD}

Bronchoscopy in patients with COPD appears to carry a greater risk compared with those with normal lung function. An increased risk of complications has been reported in severe COPD (defined as $\mathrm{FEV}_{1}<50 \%$ predicted or $\mathrm{FEV}_{1}<1 \mathrm{~L}$ and with $\mathrm{FEV}_{1} /$ forced vital capacity $\left.<69 \%\right) .{ }^{64}$ Five percent of patients with COPD compared with $0.6 \%$ of controls experienced complication: pneumonia, respiratory failure and desaturation. ${ }^{64}$ In a separate study, bronchoscopy safety was studied in patients with hypercapnia, $77 \%$ of whom had COPD. ${ }^{65}$ Desaturation occurred in $30 \%$ of the study population, $55 \%$ of patients developed wheezing and the procedure was terminated early in $20 \%$ of patients. ${ }^{65}$ A randomised control trial studied the effect of inhaled salbutamol $(200 \mu \mathrm{g})$ on $\mathrm{FEV}_{1}$ after bronchoscopy in moderate to severe COPD. ${ }^{66}$ No difference was observed (change in $\mathrm{FEV}_{1}$ ) in the group receiving salbutamol compared with placebo. Nine percent of patients experienced desaturation during bronchoscopy, with no difference again being observed between treatment groups.

In research bronchoscopy comparable complication rates have been reported. Hattotuwa et $a l^{67}$ reported a complication rate of $9 \%$, including haemoptysis (5\%), pneumothorax (2\%) and bronchospasm (2\%). All patients were given $2.5 \mathrm{mg}$ nebulised salbutamol prior to the procedure.

Evidence statements

- Patients with COPD have a higher risk of desaturation and bronchoconstriction compared with controls, however this is not a universal finding. (Evidence level 2-)

- Nebulised salbutamol administered prior to bronchoscopy does not alter the post-bronchoscopy complication rate in patients with COPD. (Evidence level 1-)

\section{Recommendations}

- COPD treatment should be optimised prior to bronchoscopy when possible. (Grade D)

- Bronchoscopists should be cautious when sedating patients with COPD. (Grade D)

\section{Patients with ischaemic heart disease}

The haemodynamic changes during FB might increase the risk of myocardial damage during the procedure. One study has shown an increased risk of ischaemic ECG changes during the procedure in patients over $60 .^{29}$ A retrospective study investigated the safety of bronchoscopy in 20 patients after acute MI. The procedure was performed an average of 12 days after MI. ${ }^{31}$ One death occurred in this study - a patient with acute myocardial ischaemia before and during the procedure. No other complications were reported. A retrospective study of patients undergoing bronchoscopy on a coronary care unit reported no difference in complications in subjects after MI compared with those without MI. ${ }^{68}$

The American perioperative guidelines recommend that elective surgery is avoided for 4-6 weeks after an acute event resulting in myocardial damage. ${ }^{69}$

Evidence statements

- Active myocardial ischaemia is a contraindication to bronchoscopy. (Evidence level 3)

- FB can increase the risk of active ischaemia, haemodynamic compromise, arrhythmia and further ischaemic events after MI. (Evidence level 3)

Recommendations

- Liaison with cardiologists should be considered in high-risk patients with cardiac disease and if FB is indicated within 4 6 weeks after MI. (Grade D)

- FB should ideally be delayed for 4 weeks after MI. (Grade D)

\section{Bronchoscopy for haemoptysis}

Bronchoscopy can be used to investigate haemoptysis. CT scans have changed the diagnostic pathway and should always be considered prior to bronchoscopy. Two studies have investigated the role of bronchoscopy following a thoracic CT scan. One study in Turkey found a bleeding site in $80 \%$ of 203 individuals even if the CT and chest x-ray were normal. ${ }^{70} \mathrm{~A}$ different study $(n=200)$ found an endobronchial diagnosis in $0.5 \%$ of individuals when the CT was normal. ${ }^{71}$

Recommendation

- Consider bronchoscopy after a normal CT if the patient is high risk for lung carcinoma or if the haemoptysis continues. (Grade D) 


\section{Bronchoscopy in the older patient}

Comorbid disease is more likely in the older patient with a potential increase in bronchoscopy risk. Several studies have shown older patients tolerate the procedure well, with no increase in complications. ${ }^{72-74}$ A prospective cohort study ${ }^{5}$ suggested that complication rates for pneumothorax and transient hypotension increased with age. When the safety of bronchoscopy was investigated in patients over 80 years old, higher complication rates and mortality rates were reported and these patients were also more likely to be mechanically ventilated after bronchoscopy. ${ }^{75}$

Recommendations

- Age alone should not be a contraindication for bronchoscopy. (Grade D)

- The older patient may require reduced doses of benzodiazepines/opioids for sedation. (Grade D)

\section{Bronchoscopy in patients who are immunosuppressed}

FB can provide useful diagnostic information when treating patients with respiratory problems who are immunosuppressed (see 'Diagnosis of infection' in this guideline for details of diagnostic value). Bronchoscopy and associated diagnostic techniques are not without risk in this patient population and this should be taken into account before performing a bronchoscopy. BAL has a reported complication rate of $0-49 \%$, depending on how the complications were defined, and deaths associated with BAL have been reported. $^{76-82}$ The main complications associated with BAL are desaturation, a drop in $\mathrm{FEV}_{1}$ and haemorrhage.

Protected specimen brushes (PSBs) can sometimes be used for similar indications to BAL. One study involved patients who were immunocompromised following bone marrow transplant ${ }^{78}$ and showed that the complication rates for PSBs were significantly higher than for BAL (36\% vs 14\%).

TBLB can carry a significant higher complication risk (30\%) compared with BAL alone. ${ }^{79} 81$ Pneumothorax, haemorrhage and desaturation were reported to be complications associated with TBLB. ${ }^{79} 81$

Three studies comparing the overall mortality in patients investigated with $\mathrm{FB}$ and patients investigated with non-invasive techniques for obtaining diagnostic samples failed to demonstrate that obtaining samples by bronchoscopy significantly reduces mortality. ${ }^{77} 78$ One study suggested that early FB to obtain diagnostic samples is associated with a lower mortality compared with delayed bronchoscopy for the same indication. ${ }^{82}$

In patients who have had lung transplantation, TBLB may be the only means of diagnosing allograft rejection. It has previously been reported that lung transplant patients have a higher risk of bleeding ( $>25 \mathrm{~mL}$ blood) (44\%) compared to other patients undergoing bronchoscopy, resulting in $5.4 \%$ of procedures being terminated early. ${ }^{36}$ Larger studies have suggested that significant bleeding occurs in $25 \%{ }^{83}$ or $13 \%$ of patients ${ }^{84}$ depending on how bleeding is diagnosed. The largest study ${ }^{85}$ suggested that bronchoscopy in lung transplant patients carried no increased risk compared with other patient groups and reported a complication risk of $0.7 \%$. It should be noted that only $57 \%$ of procedures in this study included TBLB. ${ }^{85}$ Other groups have reported higher complication rates of between $4.8 \%$ and $22 \% .^{83} 8486$ The main complications associated with TBLB are oversedation, pneumothorax and haemorrhage.

Evidence statements

- PSB has a low diagnostic yield, is rarely positive when BAL is negative and has a higher complication rate than BAL. (Evidence level 2+)
- BAL in patients who are immunocompromised has a diagnostic rate of $45-62 \%$ for infection with an associated complication rate of $0-49 \%$. (Evidence level 2++)

- TBLB carries an increased complication rate compared with BAL of around 30\%, which is mainly explained by the increased risk of pneumothorax. (Evidence level $2++$ )

- Lung transplant patients may be at higher risk of bleeding than other patients who are immunosuppressed. (Evidence level 2-)

- TBLB has a reported complication rate of between $0.7 \%$ and $22 \%$, with the main complications being oversedation, pneumothorax and haemorrhage. (Evidence level $2++$ )

Recommendations

- When a diagnosis is not likely to be obtained through noninvasive measures, bronchoscopy with BAL can be considered to provide diagnostic information. (Grade C)

- TBLB is helpful in lung transplant recipients when rejection is a possibility. (Grade $\mathrm{C}$ )

\section{PREMEDICATION, SEDATION AND TOPICAL ANAESTHESIA FOR FB \\ Premedication}

Various drugs have been considered to have a potential role as premedication, including anticholinergics (atropine and glycopyrrolate), other drugs with cardiovascular activity (eg, clonidine and labetalol), fenoterol, benzodiazepines and opioids.

\section{Anticholinergics}

Anticholinergics, such as atropine and glycopyrrolate, have been postulated to reduce cough and improve bronchoscopic views by reducing airway secretions, and also to prevent vasovagal reactions and reduce reflex bronchoconstriction. Three randomised controlled trials (cumulatively studying more than 1300 patients) examined anticholinergics and failed to demonstrate any consistent clinical benefit for patients undergoing bronchoscopy. ${ }^{87-89}$ Anticholinergics were associated with an increase in haemodynamic fluctuations (tachycardia and hypertension).

\section{Drugs with cardiovascular activity}

Hypertension and tachycardia can commonly occur during bronchoscopy. Several drugs that may blunt the cardiovascular response to bronchoscopy have been studied, postulating a role in avoidance of myocardial ischaemia and arrhythmias.

Two small randomised controlled trials examined the role of clonidine, a centrally acting antihypertensive, and demonstrated a blunting of cardiovascular responses (including blood pressure, heart rate and noradrenaline surges), although hypotension requiring treatment was seen at higher doses of clonidine and there was no improvement in patient tolerance. ${ }^{90} 91$

A randomised study of intravenous labetalol failed to demonstrate any changes in haemodynamic parameters or procedural tolerance associated with labetalol administration. ${ }^{92}$ Surprisingly, there was no tachycardia or hypertension seen in patients in the control arm (who received relatively high doses of sedation), suggesting that the sedation regime is a significant determinant of haemodynamic responses.

Further studies are required to define potential patient benefit of cardiovascular-related premedication and should be powered for endpoints such as myocardial ischaemia and arrhythmias.

Premedication with other drugs

Other small randomised controlled trials have indicated a benefit for several premedication drugs, but study limitations (size, design and lack of detailed participant characteristics) mean that further investigation is required: fenoterol may 
reduce cough rate and topical anaesthesia requirements ${ }^{93}$; dextromethorphan may lower sedation and topical anaesthesia requirements while improving patient tolerance ${ }^{94}$; low-dose oral lorazepam is associated with more favourable bronchoscopy recall at $24 \mathrm{~h}$, but not immediately after the procedure. ${ }^{95}$

The use of premedication with benzodiazepines (such as lorazepam) and opioid-like drugs (such as dextromethorphan) should be discouraged if the same class of drug is to be administered intravenously during bronchoscopy.

\section{Evidence statements}

- Three randomised controlled trials have failed to demonstrate significant clinical benefits associated with anticholinergics. Their use in bronchoscopy may be associated with an increased rate of cardiovascular adverse effects. (Evidence level $1+$ )

- There is a lack of evidence suggesting benefit of routine premedication for bronchoscopy. Small randomised studies suggest a potential role for several agents (clonidine, dextromethorphan, fenoterol, lorazepam), but such findings require validation in much larger studies of well characterised patients. (Evidence level 1-)

\section{Recommendations}

- Anticholinergics (glycopyrrolate or atropine) should not routinely be used prior to bronchoscopy due to a lack of clinical benefit and a possible increased risk of haemodynamic changes. (Grade A)

- Premedication for bronchoscopy is not routinely indicated. (Grade C)

\section{Sedation}

The majority of bronchoscopists in the UK use sedation for bronchoscopy, with only $5-10 \%$ routinely performing bronchoscopy on unsedated patients. ${ }^{96} 97$ Five randomised controlled trials, ${ }^{95}$ 98-101 one cohort study ${ }^{102}$ and one qualitative study ${ }^{103}$ examined patient and physician preference for sedation during bronchoscopy. These studies provided a broad consensus that patients and physicians usually preferred sedation for bronchoscopy, examining domains such as comfort, tolerance, bronchoscopic ease and willingness to undergo a repeat procedure. Some studies ${ }^{96} 100104$ suggested that a subset of patients tolerate unsedated bronchoscopy well, suggesting that sedation should remain an option dependent on patient preference and comorbidities (which may limit suitability for sedation).

\section{Evidence statements}

- Patients and physicians usually prefer the use of sedation for bronchoscopy. (Evidence level 1+)

\section{Recommendations}

- Intravenous sedation should be offered to patients undergoing bronchoscopy, provided there are no contraindications. (Grade B)

- Some patients will tolerate unsedated bronchoscopy well, and patient preference should be sought. (Grade B)

\section{Administration of sedation}

Sedation for bronchoscopy is usually the responsibility of bronchoscopists, although some centres use anaesthetistdelivered sedation. Bronchoscopist-delivered sedation should be carefully titrated using small incremental doses to avoid oversedation, particularly given significant variability of patient response to sedatives. The practice of non-titrated single large bolus dose sedation regimes is strongly discouraged. ${ }^{105}$

The desired depth of sedation is usually 'conscious' sedation, in which the patient maintains airway patency and cardiorespiratory function and verbal contact with the patient is possible at all times, ${ }^{106}$ although interventional bronchoscopy may occasionally require deeper sedation provided by formal anaesthetic support. Patients who are more deeply sedated should have the same level of care and monitoring as those undergoing a formal general anaesthetic. An Intercollegiate Working Party of the UK Academy of Medical Royal Colleges, chaired by the Royal College of Anaesthetists, has produced a summary of safe sedation practice (table 6).

Assessment and quantification of sedation depth may be aided by various tools, including the Ramsay Scale $\mathrm{e}^{107}$ and the Modified Observer's Assessment of Alertness/Sedation score ${ }^{108}$ (see appendix 9). These tools have particular utility in documentation of sedation level as part of the bronchoscopy record.

Evidence statement

- There is a significant and unpredictable inter-patient variability in required doses of sedative drugs. (Evidence level 1+)

Recommendations

- Sedative drugs should be titrated to provide the desired depth of sedation, given significant inter-patient variability in required doses. (Grade B)

- The desired depth of sedation is one in which verbal contact is possible at all times. (Grade D)

\section{Good practice point}

- Bronchoscopists are encouraged to document an assessment of sedation depth as part of the procedural report. $(\sqrt{ })$

Drugs used as sedatives for bronchoscopy

Please refer to appendix 8 , tables 1 and 2 .

A 2003 UK survey found that $78 \%$ of bronchoscopists routinely use midazolam sedation, with midazolam and fentanyl/ alfentanil being the most frequent combination sedation regime used. $^{96}$ Eleven randomised controlled trials ${ }^{101} 1^{108-118}$ have investigated various drugs and drug combinations for bronchoscopy sedation, including benzodiazepines (eg, midazolam), propofol (and its novel pro-drug fospropofol), ketamine and opioids (eg, fentanyl, alfentanil).

\section{Benzodiazepines and propofol}

Benzodiazepines (eg, midazolam) cause sedation, anxiolysis and anterograde amnesia by binding to and increasing the activity of

Table 6 Intercollegiate Working Party of the UK Academy of Medical Royal Colleges-summary of safe sedation practice ${ }^{106}$

\begin{tabular}{|c|c|}
\hline Domain & Safe practice \\
\hline $\begin{array}{l}\text { Patient } \\
\text { assessment }\end{array}$ & $\begin{array}{l}\text { 'Checklist' identification of sedation risk factors prior to } \\
\text { procedure. Instructions on activities subsequent to procedure } \\
\text { provided }\end{array}$ \\
\hline Level of sedation & $\begin{array}{l}\text { Sedation provided only to the level of 'conscious' sedation, } \\
\text { in which verbal contact is possible }\end{array}$ \\
\hline $\begin{array}{l}\text { Intravenous } \\
\text { sedation }\end{array}$ & $\begin{array}{l}\text { Secure venous access mandatory } \\
\text { Specific antagonist drugs should be to hand (see appendix } \\
\text { 8) } \\
\text { When combination sedation used, opioids should be given } \\
\text { first, and caution should be taken to avoid oversedation }\end{array}$ \\
\hline Monitoring & $\begin{array}{l}\text { Defined professionals should have responsibility for } \\
\text { monitoring patient safety and making a written record. Pulse } \\
\text { oximeter monitoring should be continued until discharge } \\
\text { from unit. Consider monitoring blood pressure and ECG in } \\
\text { higher risk patients }\end{array}$ \\
\hline Oxygen therapy & $\begin{array}{l}\text { Nasal cannula and facial mask oxygen delivery should be } \\
\text { available }\end{array}$ \\
\hline Facilities & $\begin{array}{l}\text { Patient trolley should be capable of being tipped head } \\
\text { down } \\
\text { Resuscitation equipment should be immediately available }\end{array}$ \\
\hline
\end{tabular}


$\gamma$-aminobutyric acid (GABA), a major brain neuroinhibitory transmitter. Other benzodiazepines (eg, diazepam and lorazepam) have been used for bronchoscopy, but midazolam has particular suitability given a rapid peak effect and a relatively short half life. Natural variability in the action of cytochrome P450 (CYP) 3A4 and 3A5, responsible for benzodiazepine metabolism, may prolong elimination half life by up to sixfold in $5-8 \%$ of the population. ${ }^{119}$

Flumazenil, a benzodiazepine antagonist, can effectively reverse benzodiazepine oversedation and must be immediately available, although its administration should not be part of routine sedation practice. ${ }^{105}$ Given that flumazenil has a shorter half life than midazolam, physicians should be aware of the risks of re-sedation and respiratory depression when flumazenil's effects cease.

A 2008 National Patient Safety Agency (NPSA) report highlighted cases of harm and death resulting from administration of excessive doses of midazolam. ${ }^{105}$ To prevent inadvertent injection of high-strength midazolam solution ( 2 or $5 \mathrm{mg} / \mathrm{mL}$ ), the NPSA mandates that only low-strength midazolam solution $(1 \mathrm{mg} / \mathrm{mL})$ should be available in clinical areas, unless a formal risk assessment has been undertaken.

Propofol (and its pro-drug fospropofol) is another sedative hypnotic that exerts its actions, in part, by increasing the activity of GABA. Together with ketamine, these drugs have a relatively narrow therapeutic window between 'conscious' sedation and general anaesthesia, and are currently recommended for use solely by anaesthetists in the $\mathrm{UK}^{106}$

Midazolam and propofol have been shown in randomised and cohort studies to improve the experience of having a FB, ${ }^{98-100}$ reduce procedural discomfort, ${ }^{98} \quad 99102$ cause anterograde amnesia, ${ }^{95} 99$ increase willingness of patients to have further procedures, ${ }^{95} 99$ without worsening the adverse event profile.

Studies comparing propofol with midazolam ( \pm opioid) have found that propofol is associated with a shorter recovery time than midazolam (although the effect size is relatively small) and both regimes are associated with similar adverse event profiles. ${ }^{109-111} 115118$ Most studies suggest that patient tolerance is broadly similar for midazolam and propofol, although two studies favoured propofol-Clark et $a l^{110}$ found a similar assessment of tolerance and cough by the bronchoscopist, but patient assessment favoured propofol. Lo et $a l^{115}$ compared bispectral index guided propofol sedation with clinically guided midazolam sedation (both with alfentanil) and found patient and physician assessment to be improved for propofol (although this group was associated with a lower mean blood pressure, of uncertain significance). Further work has compared combined propofol/alfentanil and propofol/ketamine sedation, finding high satisfaction scores for both, although worryingly, both regimes were reported as frequently being associated with oxygen saturations $<90 \%$ despite supplemental oxygen. ${ }^{114}$

\section{Opioids}

The mechanism of action of opioids such as fentanyl and alfentanil is not completely understood, but they are known to be $\mu$-opioid receptor agonists, causing analgesia, sedation and suppression of the cough reflex. Fentanyl and alfentanil have favourable pharmacological profiles for bronchoscopy, having a rapid peak effect and a relatively short half life. The competitive antagonist, naloxone, effectively reverses opioid-induced respiratory depression and oversedation. For oversedated patients who have received benzodiazepine and opioid, initial reversal with flumazenil (rather than naloxone) is recommended, unless the patient has received a particularly high dose of opioid.

Data supporting the use of monoagent sedation with opioids are limited. One small randomised study compared midazolam with alfentanil sedation, reporting a small improvement in physician-assessed cough score associated with alfentanil, with similar patient and physician assessment of discomfort and physician assessment of procedural ease. ${ }^{113}$

Addition of an opioid to midazolam or propofol improves procedural cough, reduces lidocaine usage and increases patient procedural tolerance. ${ }^{117} 118120$ The risk of oversedation may increase when using combination agents, although several studies have failed to demonstrate any increase in clinically significant adverse events. ${ }^{117} 118120$ Opioids should be administered (and allowed to reach maximal effect) prior to administration of any other agent. ${ }^{106}$

Evidence statements

- Midazolam and propofol are sedative agents that can improve bronchoscopy tolerance, decrease discomfort, improve bronchoscopic conditions and increase willingness of patients to have further bronchoscopies. (Evidence level 1+)

- Propofol is associated with a shorter recovery time and a similar adverse event profile as midazolam, although it has been evaluated in the context of medical professionals who are expert in its use. Propofol has a variable and narrow therapeutic window and higher doses cause general anaesthesia. There is no reversal agent or antagonist available for propofol. (Evidence level 1+)

- Addition of an opioid to midazolam or propofol is associated with an improvement in cough, reduced lidocaine usage and increased patient tolerance to bronchoscopy. (Evidence level $1+$ )

Recommendations

- Benzodiazepines

- Intravenous midazolam is the preferred drug for sedation, having a rapid onset of action, being titratable to provide the required depth of sedation and being reversible. (Grade B)

- No more than $5 \mathrm{mg}$ midazolam should be initially drawn up into any syringe prior to bronchoscopy for patients under the age of 70 ( $2 \mathrm{mg}$ midazolam for patients over 70$)$ to prevent potential inadvertent oversedation associated with the practice of routinely drawing up $10 \mathrm{mg}$ midazolam. (Grade D)

- Only low-strength midazolam $(1 \mathrm{mg} / \mathrm{mL})$ should be available within bronchoscopy suites. High-strength midazolam $(2 \mathrm{mg} /$ $\mathrm{mL}$ or $5 \mathrm{mg} / \mathrm{mL}$ ) should be restricted to general anaesthesia, intensive care and other areas where its use has been formally risk assessed. (Grade D)

- Propofol

- While propofol has similar efficacy to midazolam, it should only be used when administered by practitioners formally trained in its administration (eg, anaesthetists) since it has a narrow therapeutic window beyond which general anaesthesia is achieved. (Grade B)

- Opioids

- Combination opioid and midazolam sedation should be considered in patients to improve bronchoscopic tolerance. (Grade B)

- When opioids are used, short-acting agents (such as fentanyl or alfentanil) should be used to minimise post-procedural sedation. (Grade D) 
- When combination sedatives are used, opioids should be administered first and allowed time to become maximally effective before administration of any other agent. (Grade D)

Sedation in specific patient groups

Please refer to appendix 8, tables 1 and 2 .

\section{Respiratory failure}

Risk of oversedation and respiratory depression may be increased when using combined sedation for patients in respiratory failure, although one cohort study failed to demonstrate this. Dreher et $a l^{120}$ compared midazolam with combined midazolam/alfentanil sedation in 30 patients with type I or II respiratory failure. A small, but similar increase in partial pressure of $\mathrm{CO}_{2}$ was seen in both groups, and combined therapy was associated with improved patient and physician satisfaction. Nevertheless, caution is recommended when administering sedation to patients in respiratory failure.

\section{Older patients}

Older patients are likely to require lower doses of sedatives for bronchoscopy and may have prolonged after effects. A study of flunitrazepam (a benzodiazepine) found that, for the same dose of sedative, patients over 60 years old had a prolonged period of amnesia, with a extended duration of impaired coordination (which should be considered when patients are being discharged $)^{121}$ After reports of harm secondary to oversedation in older patients undergoing therapeutic gastrointestinal endoscopy, a 2004 National Confidential Enquiry into Patient Outcome and Death recommended that the practice of routinely drawing up $10 \mathrm{mg}$ midazolam should stop and no more than $2 \mathrm{mg}$ should be initially drawn up for patients over 70 years old (5 mg for patients under 70 years old). ${ }^{122} 123$

\section{Comorbidities}

Other conditions that are likely to require dose modification include hepatic impairment, heart failure and renal impairment.

\section{Concomitant medications}

Benzodiazepines, fentanyl and alfentanil are metabolised by CYP 3A4 and there is a risk of prolonged sedation in those receiving concomitant medications that inhibit these enzymes, such as antifungals, antiretrovirals, calcium channel blockers and macrolide antibiotics.

\section{Substance misusers}

A cohort study found that substance misusers are likely to need higher doses of sedatives during bronchoscopy. ${ }^{124}$

\section{Topical anaesthesia}

Methods for providing airway topical anaesthesia

Lidocaine is the most commonly used drug for topical anaesthesia of the upper airways and tracheobronchial tree, and a variety of preparations and modes of application are used. Lidocaine stabilises the neuronal membrane by inhibiting ionic fluxes, thereby preventing the initiation and conduction of action potentials. Topical anaesthesia has been achieved using 2\% gel (nasal), 10\% spray (nasal and oropharyngeal) and 1-4\% solution ('spray-as-you-go' via the bronchoscope working channel; injection into the trachea through the cricothryoid membrane; nebulised; using a dropper in the oropharynx; and applied to the nasal passages using soaked pledgets).

Lidocaine administered to the larynx and tracheobronchial tree significantly reduces incidence of cough and stridor, and reduces the requirement for sedative drugs. ${ }^{125}$ Other drugs, such as benzocaine and tetracaine, have been used, but lidocaine is the best characterised with a low risk of adverse effects (including methaemoglobinaemia) ${ }^{126}$ Cocaine has previously been used for nasal anaesthesia, but is no longer routinely available in the UK and can be associated with myocardial ischaemia and infarction. ${ }^{127} 128$

\section{Nasal topical anaesthesia}

Nasal lidocaine is most effectively provided using lidocaine gel. In the UK, 2\% lidocaine gel preparations are available in volumes of $6 \mathrm{~mL}$, although smaller volumes may be sufficient. Several studies have demonstrated that patients prefer $2 \%$ lidocaine gel to lidocaine spray, lidocaine-soaked swabs or EMLA cream. ${ }^{129-131}$

\section{Oropharyngeal topical anaesthesia}

Oropharyngeal topical anaesthesia is conventionally provided using $10 \%$ lidocaine spray (10 mg per actuation, usually two to five actuations), although nebulised lidocaine has also been used. One small randomised study ${ }^{132}$ found similar efficacy in cough suppression of $10 \%$ lidocaine spray and nebulised $4 \%$ lidocaine, although the groups were not well matched (particularly smoking status) and subsequent studies have suggested a lack of efficacy of nebulised lidocaine (see below).

\section{Laryngeal and tracheobronchial topical anaesthesia}

Several techniques may be used to administer lidocaine to the larynx and lower airways, including:

- 'Spray-as-you-go' delivery, in which lidocaine is applied via the bronchoscope working channel. Repeated application allows lidocaine delivery to the entire airway.

- Direct injection into the upper trachea using a needle passed through the cricothyroid membrane, allowing lidocaine delivery to the larynx and trachea prior to bronchoscope insertion. Further lidocaine doses to the bronchial tree may be administered as required via the bronchoscope.

Spray-as-you-go and cricothyroid lidocaine have had limited comparative evaluation. One small randomised study found a decreased cough rate, but similar patient experience, associated with cricothyroid administration (vs spray-as-you-go). ${ }^{133} \mathrm{~A}$ further small randomised study found bronchoscopic conditions, but not patient assessment, were improved for cricothyroid administration compared with nebulised lidocaine. ${ }^{134}$

The evidence for use of nebulised lidocaine is conflicting. The largest randomised controlled trial ${ }^{135}$ (150 patients) of nebulised $4 \%$ lidocaine versus saline placebo found no benefit to nebulised lidocaine (comparing additional doses of lidocaine required, and cough and comfort scores), while doubling the total dose of lidocaine administered in those who received nebulised treatment. All patients also received lidocaine spray to the nasopharynx and oropharynx, a small volume of lidocaine solution to the vocal cords and main bronchi, and sedation.

The optimal concentration of lidocaine solution has been investigated in three studies, which demonstrated that volume, rather than concentration, of lidocaine determined efficacy of airway topical anaesthesia. A randomised spray-as-you-go study, comparing the same volume of $1 \%$ and $2 \%$ lidocaine (delivered to the larynx and tracheobronchial tree), found no difference in subjective and objective measures of cough suppression. ${ }^{136}$ Another randomised study compared similar volumes of $1 \%$, $1.5 \%$ and $2 \%$ lidocaine trickled onto the back of the patient's tongue, which was allowed to be aspirated into the larynx. ${ }^{137}$ Although a relatively small study, $1 \%$ was found to be equally 
efficacious at cough suppression and had similar requirements for additional lidocaine during bronchoscopy. In a randomised awake fibre-optic intubation study, ${ }^{138}$ lidocaine was delivered using spray-as-you-go to the hypopharynx and larynx until adequate topical anaesthesia for intubation was produced. The same volume of $2 \%$ lidocaine solution was found to be as effective as $4 \%$ ( $1 \%$ lidocaine solution was not evaluated).

Evidence statements

- Topical anaesthesia with lidocaine reduces cough and decreases sedation requirements. (Evidence level 1-)

- While other topical anaesthetics such as benzocaine, tetracaine and cocaine have been studied, lidocaine is the best characterised and has a lower risk of methaemoglobinaemia. (Evidence level 3)

- Good quality randomised studies suggest lower concentration lidocaine is as effective as higher concentrations in achieving cough amelioration during bronchoscopy. (Evidence level 1+)

\section{Recommendations}

- Lidocaine should be used for topical anaesthesia during bronchoscopy, unless contraindicated. (Grade A)

- Nasal topical anaesthesia is most effectively provided using 2\% lidocaine gel. (Grade A)

- Both cricothyroid and spray-as-you-go techniques are effective in delivering lidocaine to the vocal cords and trachea. (Grade B)

- Nebulisation is not recommended as a technique for delivering lidocaine to the airways. (Grade B)

- 1\% lidocaine solution should be used for spray-as-you-go administration. (Grade A)

\section{The maximal safe dose of lidocaine}

Lidocaine has a favourable safety profile when used for bronchoscopy, although high topical doses may cause high serum concentrations, associated with subjective and lifethreatening objective signs of toxicity (particularly affecting the central nervous and cardiovascular systems-see appendix 8, table 1).

Peak serum concentrations usually occur from 20 to $45 \mathrm{~min}$ after topical application, ${ }^{137-139}$ although concentrations of active (but less potent) metabolites (eg, monoethylglycinexylidide) continue to rise for at least 2 h. ${ }^{140}$ Several studies have examined the maximal safe dose of lidocaine, with conflicting results. Lidocaine toxicity is conventionally accepted to be likely above serum concentrations of $5 \mu \mathrm{g} / \mathrm{mL}$, although studies suggest that subjective side effects may occur at much lower serum concentrations,

Frey et $a l^{141}$ studied 154 patients who received a mean dose of $15.4 \mathrm{mg} / \mathrm{kg}$ lidocaine (including nebulised lidocaine and nasal gel lidocaine). A total of $62 \%$ of patients had euphoria or dizziness (subjective signs of toxicity) despite only one patient having serum concentration $>5 \mu \mathrm{g} / \mathrm{mL}$.

Martin et $a l^{142}$ studied 39 volunteer healthcare workers having unsedated bronchoscopy who received a median dose of $9.6 \mathrm{mg} / \mathrm{kg}$ lidocaine (including nebulised and nasal gel lidocaine). A total of $92 \%$ had subjective side effects.

Other studies failed to demonstrate side effects with average lidocaine doses of 7.1-9.3 mg/kg. ${ }^{137} 139140142$ However, these data should be interpreted with caution given significant interpatient variability between dose administered and serum concentration (partly due to unpredictable absorption at different parts of the respiratory tract), with serum concentrations $>5 \mu \mathrm{g} / \mathrm{mL}$ occasionally seen even for lower doses of administered lidocaine. ${ }^{137} 140142143$ Given randomised studies suggesting that a low cough score is achievable with a total lidocaine dose of less than $160 \mathrm{mg},{ }^{118}{ }^{135}$ bronchoscopists should strive to use the lowest dose of lidocaine possible, while still ensuring good bronchoscopic conditions and patient comfort.

While lidocaine absorption varies throughout the respiratory tract, bronchoscopists should assess and record the total lidocaine dose administered during bronchoscopy (including nasal, oropharyngeal, laryngeal and tracheobronchial lidocaine). Appendix 8, table 3 illustrates the lidocaine dose associated with various formulations and concentrations.

\section{Factor influencing the risk of lidocaine toxicity during} bronchoscopy

Please refer to appendix 8 .

Risk of toxicity increases in those with hepatic and cardiac dysfunction, and significant renal impairment. Care should be taken with older patients, although one small study found no difference in adverse events associated with bronchoscopic lidocaine administration (mean dose $10.6 \mathrm{mg} / \mathrm{kg}$ ) when comparing patients aged $30-50$ years with those aged $60-75$ years. ${ }^{144}$

Evidence statements

- For the same dose of administered lidocaine, there is significant inter-patient variability in the corresponding peak serum lidocaine concentration. (Evidence level 1+)

- Doses up to $15.4 \mathrm{mg} / \mathrm{kg}$ may be used without serious adverse events, but subjective symptoms of lidocaine toxicity (eg, dizziness, euphoria) were reported in studies using $\geq 9.6 \mathrm{mg} / \mathrm{kg}$ lidocaine. (Evidence level 2+)

- Low total doses of lidocaine $<160 \mathrm{mg}$ may be associated with effective cough suppression. (Evidence level 1+)

Recommendations

- To reduce the risk of lidocaine toxicity, bronchoscopists should use the lowest dose of lidocaine sufficient to prevent excessive coughing and provide patient comfort. (Grade D)

- Bronchoscopists should remain vigilant for objective and subjective symptoms of lidocaine toxicity, particularly given significant inter-patient variability in lidocaine absorption and metabolism. (Grade B)

Good practice point

- Bronchoscopists should monitor and document the total lidocaine dose delivered at all sites during bronchoscopy. $(\sqrt{ })$

\section{SAMPLING AND DIAGNOSTIC ACCURACY}

Bronchoscopy is an important tool in the diagnostic pathway for patients with potential lung cancer, with interstitial lung disease and with pulmonary infection. A variety of sampling methods can be used during FB. A how to guide on sampling techniques can be found in appendix 10 .

\section{Lung cancer}

In patients with lung cancer, a chest CT scan should be performed prior to a bronchoscopy and patients with central lesions on the CT scan should be offered bronchoscopy if the nodal status does not influence the management decision. The diagnostic yield from bronchoscopy depends on whether the tumour is visible within the bronchial tree. Many studies have demonstrated the sensitivity of bronchial biopsy with forceps, which ranges from 48\% to 93\% (American College of Chest Physicians guidelines 2007, table 2). ${ }^{145}$

Studies have shown that the addition of bronchial brush specimens and washings increases the diagnostic yield of bronchoscopy in patients with lung cancer. The systematic review and meta-analysis carried out by the American College of Chest Physicians $^{145}$ identified 35 studies of patients with central disease undergoing bronchoscopy. The pooled data in 4507 
patients demonstrated an overall combined sensitivity of $88 \%$ for bronchoscopic techniques. Biopsy of visible lesions alone had a yield of $74 \%$ while washings had a sensitivity of $48 \%$ and yield from brushings was 59\%. McLean et al ${ }^{146}$ analysed 2238 bronchoscopies carried out between January 1991 and December 1992 in five centres in Scotland. When endobronchial tumour was seen, sensitivity for brushings and washings combined was $47 \%$. The yield from biopsy alone was $82 \%$ and when combined with brushings and washings the yield increased to $87 \%$. When the tumour was not visible the sensitivity of brushings and washings combined was $9 \% .{ }^{146}$

The optimal number of bronchial biopsy specimens in cases of visible endobronchial tumour has also been studied., Gellert ${ }^{147}$ demonstrated that at least five samples are required to achieve a $90 \%$ probability of a positive malignant biopsy for a visible tumour. ${ }^{147}$ Popovich et al ${ }^{148}$ demonstrated in peripheral lung cancer lesions biopsied using TBLB that only a $45 \%$ diagnostic yield was achieved with one biopsy and in some cases the diagnosis was not achieved before the fifth or sixth biopsy. They suggested that up to 10 biopsies may be required.

Based on recent evidence, it is reasonable to expect a diagnostic yield of $85 \%$ when the tumour is visible at bronchoscopy using bronchial biopsy. ${ }^{149} 150$ Slade $e t$ al ${ }^{149}$ examined the diagnostic yield from FB at a single centre between 2001 and 2007. In patients with tumour visible at bronchoscopy, the yield increased from $75 \%$ in 2001 to $94.5 \%$ in 2007.

The management of patients with advanced NSCLC now depends on the phenotyping and genotyping of lung cancer. ${ }^{151}$ However, the currently available evidence does not clarify whether this is routinely possible with bronchoscopic samples and in particular whether bronchial brushings and washings are suitable for subclassification of NSCLC. Studies of the sequence of biopsies, brushings and washings suggest that the order of sample acquisition is not important. ${ }^{152}$ However, to maximise the volume of tissue to allow the phenotyping and genotyping of advanced NSCLC, bronchial brushings and washings may be taken after sufficient biopsy specimens have been taken.

In addition to providing pathological information, bronchoscopy enables the location and extent of an endobronchial lesion to be determined. Careful assessment of the vocal cords is mandatory, since the presence of vocal cord palsy may provide evidence of mediastinal invasion (and thus inoperability). Bronchoscopy also allows for accurate assessment of the location and therefore $\mathrm{T}$ staging of an endobronchial tumour which may have implications for surgical treatment.

Recommendations for $\mathrm{FB}$ in performing conventional TBNA and endobronchial ultrasound (EBUS) TBNA as a diagnostic technique for lung cancer are discussed in the BTS guideline for advanced diagnostic and therapeutic FB in adults, which also includes a guide on how to perform TBNA. ${ }^{3}$

Evidence statements

- In patients with visible endobronchial tumour a diagnostic yield of at least $85 \%$ is achievable. (Evidence level 2+)

- A minimum of five biopsies should be taken when endobronchial tumour is visible. (Evidence level 3)

- The diagnostic yield from bronchoscopy can be increased by undertaking brush and lavage specimens in addition to bronchial biopsy. (Evidence level 3)

\section{Recommendations}

- A diagnostic level of $85 \%$ should be attainable when definite endobronchial tumour is visible. (Grade B)

- At least five biopsy samples should be taken when endobronchial tumour is visible to maximise diagnostic yield and the volume of biopsy tissue and to allow for tumour phenotyping and genotyping. (Grade D)

- When endobronchial tumour is visible, brushings and washings can increase the diagnostic yield of the procedure. (Grade D)

- A chest CT scan should be performed prior to a diagnostic bronchoscopy in patients with suspected lung cancer. (Grade D)

\section{Interstitial lung disease}

Bronchoscopy is a useful diagnostic tool in some ILDs, particularly sarcoidosis, hypersensitivity pneumonitis and organising pneumonia. ${ }^{153}$ BAL and TBLB are frequently performed in suspected cases of ILD.

TBLB is a technique which uses biopsy forceps to obtain alveolar tissue; it is particularly useful in obtaining histological specimens for suspected cases of ILD, although crush artefact during the procedure may render the samples inadequate for histological diagnosis, particularly in idiopathic pulmonary fibrosis. The size of forceps used does not appear to affect the diagnostic yield (see appendix 10 for a step-by-step guide on how to perform TBLB). ${ }^{153}$ A retrospective review of 452 bronchoscopies in localised and diffuse lesions examined by TBLB demonstrated a diagnostic yield in $55.2 \%$ of patients with a localised lesion and $67 \%$ in those with diffuse lesions. The study also demonstrated an increase in the diagnostic yield with an increasing number of specimens, so that taking more than four biopsies increased the yield from 52\% to more than $70 \% .{ }^{43}$ The complication rate in this series was $6 \% ; 5.8 \%$ developed a pneumothorax $(3.8 \%$ required intercostal drainage) and significant bleeding was noted in $0.2 \%$, suggesting that TBLB is a useful diagnostic procedure with a low complication rate $^{43}$

The use of BAL may be controversial, since its use in diagnosis and monitoring disease activity is limited in ILD, and its current strength is in research in understanding the underlying pathophysiological mechanisms in ILD. ${ }^{154}$ There is a suggestion that combining techniques increases diagnostic yield. In a study investigating patients with sarcoidosis, TBLB alone was diagnostic in 58\%, TBNA alone was successful in diagnosing $17 \%$ of cases as was BAL alone; combining all three procedures led to a $100 \%$ diagnostic rate. ${ }^{155}$

No evidence currently exists for the coexistent use of BAL with TBLB, however it is reasonable to perform both these procedures in cases of suspected ILD. BAL and TBLB should be performed in areas shown to be affected on high-resolution CT $(\mathrm{HRCT})^{156}$ and both procedures should be performed on the same lung. In those with typical HRCT features of idiopathic pulmonary fibrosis, BAL and TBLB are not required for diagnosis.

Diagnostic bronchoscopic procedures have moderate (67-74\%) diagnostic sensitivity in patients with diffuse ILD, ${ }^{157} 158$ but have high diagnostic sensitivity in cases of sarcoidosis. In a study of 530 consecutive TBLBs in patients with diffuse infiltrates, the overall diagnostic yield was 50\% and $75 \%$ for stage II-III sarcoidosis. The diagnostic yield of hypersensitivity pneumonitis in this series was $92 \%$. In a case series of 183 patients with bilateral diffuse shadowing, Mitchell et al ${ }^{159}$ demonstrated that TBLB had a diagnostic yield of $77 \%$ for patients with sarcoidosis.

The diagnostic sensitivity of TBLB and BAL in sarcoidosis is estimated to be between $56 \%$ and $77 \%$, increasing with the severity of the disease. ${ }^{157}{ }^{159-164}$ One study demonstrated the sensitivity of TBLB to be $55 \%$ in stage I disease, $80 \%$ in stage II 
disease and $82 \%$ in stage III disease. ${ }^{162}$ In sarcoidosis, EBB may also prove useful, even without visible endobronchial abnormality, particularly when parenchymal disease exists. ${ }^{163}$ Combining techniques will improve diagnostic yield. ${ }^{160}{ }^{161}$ Mediastinal lymphadenopathy can be sampled by conventional or ultrasound guided TBNA-please refer to the BTS guideline for advanced diagnostic and therapeutic FB in adults for specific recommendations and evidence. ${ }^{3}$

A minimum of five to six TBLB samples should be taken and increasing the number of samples will increase the diagnostic yield, particularly in those with a localised lesion. ${ }^{164}$ A direct correlation has been shown between the number of TBLBs taken and diagnostic yield, with a $35 \%$ diagnostic yield for one to three biopsies, increasing to 69\% with 6-10 biopsies. ${ }^{164}$ Furthermore, Popovich et al ${ }^{148}$ demonstrated in peripheral lung cancer lesions biopsied using TBLB that only a $45 \%$ diagnostic yield was achieved with one biopsy and in some cases the diagnosis was not achieved before the fifth or sixth biopsy. They suggested that up to 10 biopsies may be required.

There is conflicting evidence regarding the use of fluoroscopy during TBLB. A retrospective review by Anders et al of 112 TBLBs performed with fluoroscopy and 135 without fluoroscopy found no significant difference in the yield $(72.3 \%$ vs $67.4 \%$ ); however the yield for localised lesions was lower without fluoroscopy. ${ }^{165}$ Fluoroscopy may be useful when performing TBLB on localised peripheral lesions. The diagnostic rate of TBLB without fluoroscopy varies between $60 \%$ and $65 \%,{ }^{159} 166$ and with fluoroscopy $55.2^{-73} \%{ }^{43} 165$ for localised lesions. It has therefore been suggested that fluoroscopy may be useful when performing TBLB on localised peripheral lesions. Two studies have demonstrated that knowledge of the location of the lesion from chest radiograph aids diagnostic yield. To ensure that peripheral localised lesions are being accessed correctly, fluoroscopy may therefore be required. ${ }^{43} 167$ The incidence of pneumothorax following TBLB without fluoroscopy is between 3\% and 5\%, ${ }^{166} 168$ although one study demonstrated no increase in the incidence of pneumothoraces $(0.015 \%) .{ }^{157} \mathrm{~A}$ chest $\mathrm{x}$-ray may be performed immediately if pneumothorax is suspected or the patient is symptomatic, or be done at least $2 \mathrm{~h}$ after TBLB to exclude pneumothorax. ${ }^{47}$

Evidence statements

- EBBs should be performed in patients with suspected sarcoidosis. Yield increases in patients with radiographic evidence of parenchymal disease. (Evidence level 2+)

- TBLBs should be performed in patients with radiographic stage II-IV sarcoidosis. (Evidence level 2+)

- At least five to six TBLBs should be performed in patients with ILD. (Evidence level 2-)

- There is no convincing evidence that the use of fluoroscopy reduces pneumothorax rate or diagnostic yield in non-focal lung disease. (Evidence level 3)

Recommendations

- In suspected sarcoidosis, EBBs should be considered to increase the diagnostic yield. (Grade C)

- TBLB is recommended for the diagnosis of stage II-IV sarcoidosis. (Grade C)

- In patients with diffuse ILD, five to six TBLBs should be taken from the same lung. (Grade D)

- Fluoroscopy should be considered for TBLB in patients with localised or focal parenchymal lung disease. (Grade D)

Good practice point

- Bronchoscopists should keep a record of their personal diagnostic accuracy for FB. $(\sqrt{ })$

\section{Diagnosis of infection}

Bronchoscopy is a useful procedure to diagnose infection when less invasive methods to diagnose the underlying aetiology are not suitable.

This section includes basic sampling methods (bronchial wash, BAL, TBLB, EBB and bronchial brush) but does not include advanced techniques such as EBUS TBNA, which are covered in the advanced bronchoscopy guidelines. ${ }^{3}$

The spectrum of infections encountered in immunocompromised hosts differs from that seen in immunocompetent individuals. The role of FB in obtaining diagnostic samples in these two patient groups also differs

\section{Bronchoscopy in the diagnosis of infection in the} immunocompromised host

The underlying aetiology for pneumonia in patients who are immunocompromised varies with the prevalence of disease in the community and with geographical area. Studies performed in Africa where there is a high prevalence of TB have shown the most common organism causing pneumonia in patients with HIV-related immunosuppression is mycobacterium TB. ${ }^{169}{ }^{170} \mathrm{In}$ Western countries with a lower prevalence of $\mathrm{TB}$, commonly encountered organisms are Pneumocystis jiroveci, fungal infections, bacterial infections and viral infections such as cytomegalovirs and Cryptococcus. ${ }^{171-173}$ A variety of bronchoscopic techniques can be used depending on the local population, disease prevalence and host defences (figure 1).

\section{Bronchoalveolar lavage}

BAL has been shown to have high sensitivity in $P$ jiroveci pneumonia. In the absence of prior antibiotic use for treatment or prophylaxis, BAL is reported to have a sensitivity between $90 \%$ and $98 \%$ for $P$ iroveci. ${ }^{174-179}$ Prior use of empiric antibiotic treatment reduces the sensitivity to around $64 \% .{ }^{178}$ Bilateral BAL sampling has a higher diagnostic yield compared with unilateral BAL ${ }^{179}$ and BAL from upper lobes has higher sensitivity compared with lower or middle lobe sampling techniques. ${ }^{180} 181$ The comparable sensitivity of BAL to transbronchial biopsy, ${ }^{176}$ but with fewer inherent risks, means that BAL is regarded as the gold standard investigation for suspected $P$ jiroveci infection.

BAL in patients who are immunocompromised with smearnegative pulmonary $\mathrm{TB}$ has been reported to have a sensitivity of $10-30 \%$ based on microscopy ${ }^{182-184}$ and a sensitivity of $52-95 \%$ on culture. ${ }^{182-184}$ When PCR techniques are used on BAL material, a rapid diagnosis of $\mathrm{TB}$ with reported sensitivity of $85.7 \%$ and specificity of $90.9 \% \%^{185}$ have been reported. Serological tests on BAL in patients with HIV (unlike those without HIV) with suspected pulmonary TB have shown a lower sensitivity, but the specificity remains high. ${ }^{185}$

In invasive aspergillosis, BAL is reported to show characteristic hyphae in $34-64 \%{ }^{186-188}$ and to be positive on cultures for Aspergillus in $23-85 \%$ of cases. ${ }^{186-188}$ If available, galactomannan antigen detection on BAL is useful. A meta-analysis looking at the performance of BAL galactomannan testing in detection of proven invasive aspergillosis suggested that the test has a sensitivity of $94 \%$ and a specificity of $79 \% .{ }^{189}$ Unlike the serum galactomannan test, BAL galactomannan is equally sensitive in patients with haematological or other causes of immunosuppression. ${ }^{190}$ There have been reports of false-positive results when using the serum galactomannan test, especially in patients who are receiving certain $\beta$-lactam antibiotics. ${ }^{191-194}$ The reported sensitivity for PCR on BAL in cases of invasive aspergillosis is $67-100 \%$ and the specificity $96-100 \% .^{195} 196$ The PCR 


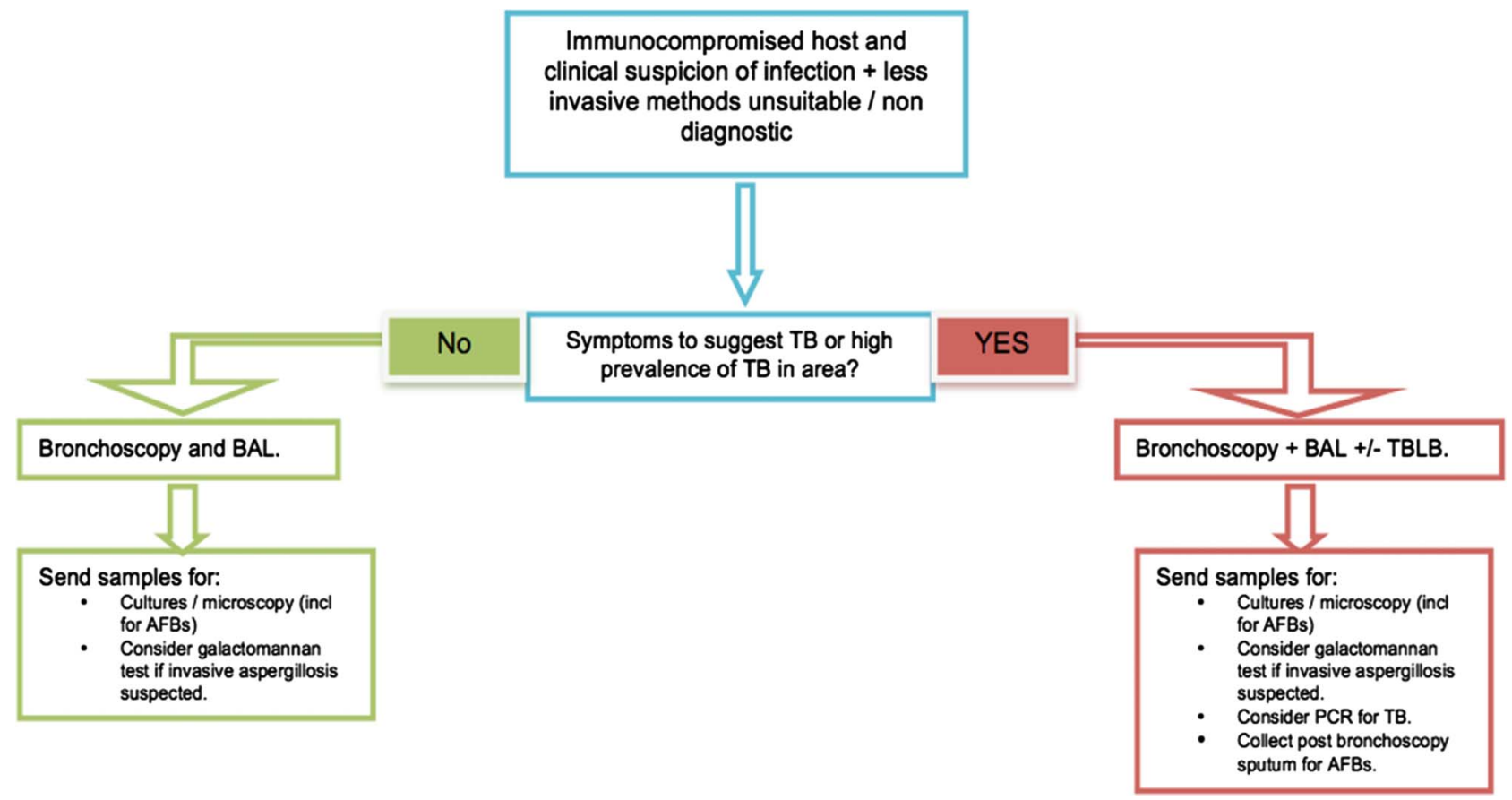

Figure 1 Diagnostic algorithm: bronchoscopy in the immunocompromised host.

techniques have some limitations, including lack of standardisation for processing of samples.

BAL and bronchial washings in patients who are immunocompromised with bacterial pneumonia have been shown to change the diagnosis in 50\% and change antibiotic therapy in $62 \%$ of patients who are HIV positive. ${ }^{197}$ In patients with AIDS and suspected cryptococcal disease, bronchoscopies with BAL and bronchial washings have a combined sensitivity on smear equal to that of TBLB and superior to that of TBLB fungal culture. $^{198}$

\section{$T B L B / E B B$ and brush biopsies}

TBLB has been reported to have a comparable yield to BAL in cases of $P$ jiroveci pneumonia in some studies, ${ }^{171} 199200$ although other studies have shown it to be inferior ${ }^{175} 176$ and some have shown it to have higher sensitivity than BAL. ${ }^{177}$ Since TBLB carries the risk of pneumothorax of between $2.5 \%$ and $3.7 \%,{ }^{176} 177$ it is not recommended in patients with suspected $P$ jiroveci pneumonia. In areas of low TB prevalence where Pneumocystis is more likely to be responsible for infection, ${ }^{173}$ TBLB may not be required during the initial bronchoscopy.

In cases of pulmonary TB in immunocompromised hosts, the sensitivity of TBLB based on positive microscopic examination is reported to be between $19 \%{ }^{183}$ and $39 \% .{ }^{182}$ Although fewer granulomata are seen on TBLB in patients who are immunocompromised compared with those who are immunocompetent, TBLB was the reported exclusive method of diagnosis in around $13 \%$ of patients in a study. ${ }^{183}$ The overall reported sensitivity for cultures of TBLB is reported to be $42-52 \% .^{182-184}$

EBB and TBLB have a lower diagnostic yield compared with BAL in the diagnosis of invasive aspergillosis. ${ }^{197} 201$ Biopsy may confirm the presence of hyphae and demonstrate pathognomonic tissue damage due to hyphae. Careful consideration must therefore be given to the risk versus benefit, especially the risk of bleeding if concurrent thrombocytopenia is present, or of pneumothorax in these critically ill patients.
Brush biopsies to diagnose $P$ jiroveci pneumonia have been consistently found to have lower sensitivity compared with BAL, with reported sensitivity between 29\% and $52 \% .{ }^{171} 175176199$ Due to its limited sensitivity, brush biopsy has a limited role in this setting.

Evidence statements

- BAL has excellent yield in P jiroveci pneumonia and is now considered the gold standard. (Evidence level 3)

- BAL has a good diagnostic yield for the majority of infections and may be sufficient as the only bronchoscopic investigation in countries with a low prevalence of TB. (Evidence level 3)

- BAL can show characteristic hyphae and has moderate sensitivity for invasive aspergillosis based on cultures.(Evidence level 3)

- BAL galactomannan test has excellent sensitivity and specificity for invasive aspergillosis and should be considered if available. (Evidence level 3)

- Post-bronchoscopy sputum is a complementary method of collecting material for staining for AFB and should be collected if the patient is able to provide a sample. (Evidence level 3)

Recommendations

- In patients with pulmonary infiltrates who are immunocompromised and in whom TB is considered unlikely, BAL alone is usually sufficient to achieve a diagnosis. In areas or populations with high prevalence of TB, TBLB may be considered in addition. (Grade C)

- BAL or bronchial washings should be sent for microscopy for AFB and for mycobacterial culture in patients with pneumonia who are immunocompromised. (Grade C)

- Post-bronchoscopy sputum could be collected in patients who are immunocompromised and suspected to have TB. (Grade D)

- TBLB and EBB for invasive aspergillosis may be avoided if BAL galactomannan test is available due to the high sensitivity and specificity of the latter and inherent risks with the biopsies. (Grade C) 
- In patients suspected to have invasive aspergillosis, BAL should be sent for microscopy for hyphae and fungal culture; a BAL galactomannan test should be considered to further improve diagnostic yield. (Grade C)

\section{Bronchoscopy in the diagnosis of infection in the} immunocompetent host

There are no randomised trials to compare the outcome of invasive tests such as bronchoscopy in the early phase of infection with non-invasive tests and/or empirical treatment in cases of community-acquired pneumonia. The current guidelines on management of community-acquired pneumonia recommend the use of non-invasive investigations. In patients with moderate to severe disease, bronchoscopy should be reserved for patients whose condition fails to respond. ${ }^{202} 203$ The role of bronchoscopy in the early phase of management of a patient with community-acquired pneumonia may be quite limited.

Bronchoscopy should be considered in patients whose condition fails to respond to initial treatment for community-acquired pneumonia. In this setting, FB can help to exclude underlying lung cancer which can be present in a significant proportion of patients with non-resolving pneumonia ${ }^{204} \mathrm{FB}$ is also useful in this patient group to diagnose and remove aspirated organic or inorganic material. In adults, the presence of a non-resolving pneumonia, lung abscess, atelectasis or collapse can indicate possible aspiration. ${ }^{205} \mathrm{FB}$ is the first-line investigation of choice in adults to remove aspirated material with reported success rates of up to $100 \%$ in experienced hands. ${ }^{205} \mathrm{~A}$ bronchoscopy in patients whose condition has failed to respond to treatment provides an opportunity to collect additional samples for microbial cultures.

In patients who are suspected to have TB but are unable to provide an adequate sputum sample, bronchoscopy can be used as an alternative to induced sputum to obtain material for staining. The overall combined yield of bronchoscopy in patients with negative sputum smears, with procedures such as BAL, bronchial biopsies, bronchial wash and post-bronchoscopy sputum, is reported to be $28.8-48.3 \%{ }^{206} 207$ based on microscopy and smears for AFB and $69.2-94 \% 206208209$ based on cultures.

\section{$B A L$ and bronchial aspirate}

Bronchial aspirates obtained for the purpose of culture and sensitivity have a significant false-positive rate due to contamination with upper respiratory tract secretions. ${ }^{210}$ Protected catheters have a lower risk of contamination. ${ }^{211}$ Protected catheter and BAL samples have a reported sensitivity based on quantitative cultures of $64.7-70 \%$ and $73.8-77 \%$ respectively in patients with community-acquired pneumonia who had a bronchoscopy within $12 \mathrm{~h}$ of hospital admission. ${ }^{212} 213$ The yield is lower when bronchoscopy is performed after treatment failure (lack of response after $72 \mathrm{~h}) .^{214}$

BAL also provides suitable material for other tests such as PCR and antigen detection. Performing the pneumococcal antigen test on BAL is reported to have a sensitivity of $54-95 \%$ and a specificity of $86.8-100 \%$ for pneumococcal pneumonia. ${ }^{215} 216$ When available, use of PCR on BAL has been shown to be useful in the detection of legionella, ${ }^{217} 218$ with reported sensitivity and specificity approaching $100 \% .^{218}$ It has been suggested that samples should routinely be sent for legionella PCR if bronchoscopy is performed. ${ }^{202}$

In patients with suspected $\mathrm{TB}$ and negative sputum smear, BAL and bronchial aspirates range in sensitivity from $23 \%$ to $28 \%$ on smears ${ }^{207} 219$ and $32-88 \%$ on cultures. ${ }^{207} 208219$ Studies have shown a high sensitivity of PCR when performed on BAL fluid with a range of $78-80.9 \% .^{220} 221$ The ability to make an early diagnosis by using PCR can be further enhanced by performing BAL smears at the same time. ${ }^{221}$ BAL PCR appears to have a false-positive rate of around $4.4 \% .^{220}$ Tests on BAL, such as enzyme-linked immunospot have high sensitivity and specificity and can rapidly distinguish between smearnegative pulmonary TB and latent TB infection. ${ }^{222} 223$

\section{$E B B$ and $T B L B$}

EBB or TBLB are useful in the diagnosis of slowly or nonresolving pneumonia if there are any visible endobronchial abnormalities or if there is a possibility of TB. There is no evidence to suggest they are useful as a routine test in patients with pneumonia.

In patients with proven pulmonary $\mathrm{TB}$, bronchial and brush biopsies show typical granulomata in 3.84-53\% of cases. $^{206} 219224$ Post-bronchoscopy sputum is culture positive in $14-46 \%$ of patients and sputum should be cultured if the patient is able to provide an adequate sample. ${ }^{207} 208$

Evidence statements

- A significant proportion of patients (11\%) with non-resolving pneumonia, who are over 50 and are ex-smokers or current smokers, may have underlying carcinoma. Bronchoscopy can play an important role in diagnosing endobronchial lesions, especially if there is an additional history of weight loss of $>3 \mathrm{~kg}$ in the preceding 3 months. (Evidence level 2-)

- PCR for legionella on BAL specimens has a sensitivity approaching 100\%. (Evidence level 2+)

- In TB, BAL, bronchial aspirates and post-bronchoscopy sputum all contribute to the overall diagnostic yield. (Evidence level 2+)

- In high-prevalence areas, routine samples for culture and microscopy for TB confirm TB in 6-8\% of patients, even if bronchoscopy is performed for another indication. (Evidence level 2+)

Recommendations

- Bronchoscopy may be considered in patients with nonresolving or slowly resolving pneumonia, especially if they are current or ex smokers and older than 50 years. (Grade C)

- If bronchoscopy is performed for community-acquired pneumonia, BAL specimens should be sent for legionella PCR and atypical pathogens. (Grade C)

- Bronchoscopy may be considered if the patient is suspected to have TB when sputum smear is negative. (Grade $\mathrm{C}$ )

- In cases of suspected TB, BAL, bronchial aspirates, and postbronchoscopy sputum appear to be complementary and should all be analysed. (Grade C)

- In areas with high or intermediate prevalence of TB, patients undergoing bronchoscopy for another indication should have samples sent routinely for cultures for TB. (Grade C)

\section{BRONCHOSCOPY IN THE ICU}

FB facilitates inspection of the upper airways and bronchial tree in critically ill patients in the ICU. In non-intubated patients the risks of bronchoscopy are those described in the section on 'Monitoring, precautions and complications'. However, bronchoscopy in the ICU commonly involves intubated patients supported by mechanical ventilation, with significant other illness and comorbidities, and therefore bronchoscopy requires careful consideration in this patient population.

\section{Bronchoscopes used in the ICU}

The specifications of a bronchoscope suitable for use in the ICU require a degree of compromise. The internal diameter of the 
ET may restrict the size of the bronchoscope, while efficient suctioning requires a larger bronchoscope with a wide suction channel. The internal diameter of the tracheal tube relative to the external diameter of the bronchoscope is an important consideration. Bronchoscopes in the non-intubated patient occupy only $10-15 \%$ of the cross-sectional area of the trachea. In contrast, a $5.7 \mathrm{~mm}$ bronchoscope occupies $40 \%$ of a $9 \mathrm{~mm}$ ET and $66 \%$ of a $7 \mathrm{~mm}$ tracheal tube. Failure to recognise this may lead to inadequate ventilation of the patient and impaction of or damage to the bronchoscope.

Lubrication is essential to facilitate passage of the bronchoscope. Tracheostomy tubes are prone to damage the bronchoscope, particularly during withdrawal when the rigid edge of the end of the tracheostomy tube can abrade the covering of the bronchoscope.

A single model (non-human) study addresses the issue of airway support device and size of bronchoscope. ${ }^{225}$ In this study, the laryngeal mask airway (LMA), reinforced LMA (RLMA) and ET were assessed in a simulated model of ventilation, measuring flow resistance during simulated inspiration and the maximum size of flexible bronchoscope (using oesophageal dilators to mimic six different sizes of bronchoscope (2.7-8.9 mm external diameter)). This study demonstrated that the LMA can accommodate a larger bronchoscope than the corresponding sizes of RLMA and ET. Mean flow resistance was 2.3 (1.7-3.5) times higher with the RLMA and 2.1 (1.2-4.2) times higher with the ET compared with the LMA, and 1.2 (0.7-1.8) times lower with the ET compared with the RLMA. Removal of the LMA aperture bars resulted in a mean decrease in flow resistance of $3.6 \%$. The study provides useful information on the maximum external diameter of the bronchoscope to be used with different airway devices. This information is provided in appendix 11 of this guideline.

\section{Recommendation}

- The external diameter of a bronchoscope used in the ICU setting should be carefully selected according to the external diameter of the bronchoscope, the size of the airway support device (ET or laryngeal mask) and the type of airway device used. (Grade D)

\section{Indications for FB in the ICU}

FB may be performed in the ICU for a wide range of diagnostic or therapeutic indications. These are considered separately in the sections below.

\section{Treatment of lobar collapse}

Bronchoscopy has been used to treat lobar collapse in intubated patients whose condition has failed to respond to treatments such as physiotherapy. Retained bronchial secretions may obstruct major airways, and local directed suction using a wide channel bronchoscope combined with saline instillation has been used to treat this condition.

A total of four case series ${ }^{226-229}$ (totalling 47 patients) and one case report ${ }^{230}$ have reported the use of bronchoscopy in ventilated patients to reverse lobar atelectasis, with good immediate outcome reported in all studies. All such studies are noncomparative and therefore subject to bias. However, bronchoscopy may be considered in patients with lobar atelectasis which is not responding to conventional treatment.

One randomised trial has been conducted assessing the utility of bronchoscopy in the prevention of atelectasis in 29 patients, ventilated after thoracic surgery (lobectomy). ${ }^{231}$ In this study, there was no difference in any outcome measured (including blood gases, spirometry, the need for further bronchoscopy, radiology, ICU and hospital stay) between those treated using bronchoscopy and those treated with suction and physiotherapy (standard care).

Evidence statement

- A well conducted randomised trial demonstrates no benefit in the use of bronchoscopy compared with physiotherapy and suction in the prevention of post-lobectomy atelectasis in ventilated patients. (Evidence level 1+)

Recommendations

- Prophylactic bronchoscopy and lavage should not be used to prevent post-lobectomy atelectasis in ventilated patients. (Grade A)

- Bronchoscopy may be considered in specific circumstances for the relief of atelectasis in intubated and ventilated patients. (Grade D)

\section{Diagnosis and management of haemoptysis/haemorrhage}

A minor degree of endotracheal bleeding is common during routine tracheal suctioning and may result from tracheal epithelial abrasions. If an intubated patient has persistent or excessive bleeding through the ET, bronchoscopy may identify the source and extent of the haemorrhage.

A total of two case series report the utility of bronchoscopy in the management or diagnosis of haemoptysis/haemorrhage in ventilated patients. $^{232} 233$ A case series of seven patients reports control of haemorrhage in six of seven patients using bronchoscopic intervention (saline/adrenaline instillation) after an average of 5.9 treatments per patient, with one patient reported as dying of haemorrhage. ${ }^{232}$

A larger case series has retrospectively compared the utility of bronchoscopy and CT in the diagnosis and management of haemoptysis in 80 cases of haemoptysis in ventilated patients. ${ }^{233}$ A bleeding site was identified with bronchoscopy in 71 (89\%) vs $64(80 \%)$ with HRCT ( $p>0.3)$. The two techniques together diagnosed the site of bleeding in $96 \%$. The final cause of haemoptysis was identified by bronchoscopy in only two patients, compared with 49 patients using HRCT.

CT is therefore likely to be the first-line diagnostic test in haemoptysis/haemorrhage in ventilated patients. Bronchoscopy may be diagnostically useful in specific circumstances when haemoptysis occurs in mechanically ventilated patients (eg, for the diagnosis of diffuse alveolar haemorrhage on bronchoscopic samples), but there is no evidence that directly addresses this issue. FB may be used in the management of haemoptysis in mechanically ventilated patients, but the evidence for its efficacy is scanty, and consideration should be given to local resources and other feasible techniques (eg, rigid bronchoscopy, surgery or interventional radiology) depending on the clinical situation.

\section{Recommendation}

- Bronchoscopy may be considered in ventilated patients with haemoptysis if CT imaging has been performed and is unhelpful, or is not possible. (Grade D)

\section{Diagnosis of infection in ICU/ventilated patients}

Bronchoscopically directed lavage or brushing techniques and 'non-invasive' techniques (blind catheter brushes) can be used to obtain microbiological samples in ventilated patients with suspected ventilator-associated pneumonia. Either qualitative or quantitative microbiological techniques can be used to analyse the samples obtained.

There are a large number of case series assessing the utility of bronchoscopy in the diagnosis of infection in ventilated patients ${ }^{234-257}$ which show great variability in the gold standard 
used for the diagnosis of ventilator-associated pneumonia (autopsy, clinical course, bronchoscopy results), the microbiological cut-off threshold for the diagnosis of confirmed infection (number of colony-forming units, number of intracellular organisms) and the exact bronchoscopic and non-bronchoscopic techniques used (BAL, protected telescope brush, telescoping catheters). This results in a wide range of reported sensitivities of bronchoscopic techniques (51-100\%), and variable comparison to non-invasive techniques. Overall, bronchoscopy and lavage or brushing appears to be safe for microbiological diagnosis in ventilated patients.

A well conducted systematic review conducted in $2008^{258}$ and then repeated in $2012^{259}$ included five randomised trials (totalling 1367 patients) which assessed the utility of invasive (bronchoscopic) versus non-invasive (blind catheter brush and other blind techniques) in reducing mortality and ICU stay in ventilated patients with clinically diagnosed ventilator-associated pneumonia. Comparing quantitative and qualitative cultures (1240 patients), there were no statistically significant differences in mortality rates $(\mathrm{RR}=0.91,95 \% \mathrm{CI} 0.75$ to 1.11$)$. In addition, there was no evidence of mortality reduction in the invasive group versus the non-invasive group $(\mathrm{RR}=0.93$, 95\% CI 0.78 to 1.11) and no significant differences between the interventions in the number of days on mechanical ventilation, length of ICU stay or antibiotic change.

Thus, evidence from a well conducted systematic review ${ }^{259}$ suggests that bronchoscopic (invasive) techniques are not superior to less invasive techniques as a diagnostic strategy in ventilated patients with suspected pneumonia, with no improvement in clinically meaningful outcomes (mortality, ICU stay, days ventilated) using more invasive diagnostic strategies. As bronchoscopic techniques are more invasive with no demonstrated benefit over less invasive techniques, this evidence suggests bronchoscopy should not be used in preference to non-invasive diagnostic strategies. However, when non-invasive diagnostic strategies are not available, bronchoscopy to confirm diagnosis may be a reasonable option, although there is no direct evidence to address this issue.

The evidence above does not address the issue of diagnosis of non-ventilator-associated pneumonia in ventilated patients (eg, patients with community-acquired pneumonia who subsequently require ventilation).

Evidence statement

- In the diagnosis of ventilator-associated pneumonia, invasive diagnostic strategies using bronchoscopy are not associated with improvement in clinically important outcomes in ventilated patients (mortality, length of ventilation and length of ICU stay) compared with 'non-invasive' techniques. (Evidence level 1-)

\section{Recommendations}

- Directed non-invasive diagnostic strategies (eg, blind catheter aspiration) should be used first line in preference to bronchoscopy in ventilated patients with suspected ventilatorassociated pneumonia. (Grade A)

- When such non-invasive diagnostic techniques fail to identify a responsible organism, bronchoscopy should be considered for the diagnosis of ventilator-associated pneumonia. (Grade D)

Transbronchial biopsies and needle aspiration in the ICU/ ventilated patients

Transbronchial biopsy may be considered in selected patients for histological diagnosis. A single case series reports the utility of transbronchial biopsy under fluoroscopy in 13 ventilated patients with progressive infiltrates. ${ }^{260}$ Transbronchial biopsy established the diagnosis in six (46\%), and was considered helpful in treatment of all these patients. However, significant complications occurred in 31\% (two pneumothoraces requiring drainage, one haemorrhage and one arrhythmia), although no deaths were attributed to the procedure. On the basis of this limited evidence, transbronchial biopsy should be cautiously used in intubated/ventilated patients, with poor diagnostic yields $(<50 \%)$ and significant potential harm.

A small (eight patient) retrospective case series described the use of TBNA in ventilated patients with abnormal lymph nodes identified on CT scan. ${ }^{261}$ In this series, there were no complications, and discounting a single case in which a definitive diagnosis could not be obtained, TBNA had a sensitivity of $83 \%$ and a specificity of $100 \%$ (giving positive predictive value of $100 \%$, negative predictive value of $50 \%)$. In five of eight (63\%) cases, TBNA led to a change in management.

This small case series suggests that TBNA may be a useful procedure in highly selected mechanically ventilated patients.

\section{Diagnosis of acute respiratory distress syndrome}

A randomised crossover study has been conducted comparing the diagnostic utility of bronchoscopy and catheter lavage in differentiating acute respiratory distress syndrome (ARDS) $(n=21)$ from those at risk of ARDS $(n=20)$ using cell profiles. ${ }^{262}$ Although this study was well conducted and demonstrated that bronchoscopy and BAL can distinguish between ARDS and acute lung injury when catheter lavage cannot, the diagnostic methods used (total protein, protein permeability, epithelial lining fluid volume and myeloperoxidase) are research tools and not in current standard practice, and the clinical implications are unknown.

\section{Precautions and contraindications to bronchoscopy on the ICU}

Critically ill patients represent a high risk group for most invasive procedures. They will often present with hypoxia, electrolyte disturbances, clotting abnormalities and arrhythmias. It is therefore important that the potential benefits of bronchoscopy outweigh the risks. Elevated prothrombin time, increased activated partial thromboplastin time, reduced fibrinogen titre or thrombocytopenia indicate clotting dysfunction which makes biopsy procedures hazardous. Critically ill patients may be more susceptible to the toxic effects of local anaesthetics. However, in the ventilated patient intravenous sedation or anaesthesia is probably the most appropriate alternative.

A case series of 37 ventilated patients with severe thrombocytopenia (platelets $<50$ ) undergoing bronchoscopy (33 BALs, 5 TBLBs) ${ }^{263}$ demonstrated only two deaths associated with the procedure $(5.4 \%)$, one of which was secondary to a major airway bleed. Minor tracheobronchial bleeds requiring no treatment were seen in $32 \%$. In this study, it is not clear which patients required platelet transfusions. However, overall, it would appear that bronchoscopy in patients with low platelets is feasible (if corrected) but associated with significant potential harm.

In a case series of 110 mechanically ventilated, highly selected patients with ARDS on standard criteria (excluded if partial pressure of $\mathrm{O}_{2}<80 \mathrm{~mm} \mathrm{Hg}$ on $\mathrm{FiO}_{2}$ of 1.0 , hypotension, ischaemic heart disease, raised ICP or ET $<7 \mathrm{~mm}$ diameter), bronchoscopy and BAL appeared to be relatively safe in the short term $(1 \mathrm{~h})$ with no clinically significant changes in oxygenation. $^{264}$ One episode of pneumothorax (without transbronchial biopsy) occurred. Thus bronchoscopy and BAL appear to be 
safe in the short term in highly selected patients with ARDS in terms of oxygenation.

\section{Recommendations}

- Patients in the ICU should be considered at high risk from complications when undergoing bronchoscopy. (Grade D)

- All potential risk factors (ventilator parameters, clotting dysfunction) should be corrected as far as possible before undertaking bronchoscopy. (Grade D)

Good practice point

- The risks and benefits of bronchoscopy should be carefully considered in mechanically ventilated patients. $(\sqrt{ })$

\section{Monitoring}

A full range of physiological monitoring is available in the ICU, including ECG (for heart rate and rhythm), continuous intra-arterial blood pressure or intermittent cuff blood pressure measurement, pulse oximetry $\left(\mathrm{SpO}_{2}\right)$ and end-tidal $\mathrm{CO}_{2}$ monitoring. Setting appropriate alarm limits for heart rate, blood pressure and $\mathrm{SpO}_{2}$ and requesting other attendant staff to monitor physiological variables during the bronchoscopy allows close monitoring for deterioration during the procedure. Adverse events require immediate withdrawal of the bronchoscope and resuscitation of the patient. The clinician must then weigh the benefits against the risks of proceeding further.

A total of 13 studies have addressed potential physiological parameters which may be altered by bronchoscopy in ventilated/ ICU patients. ${ }^{239} 243246263-271$ A randomised trial of different BAL volumes using bronchoscopy in mechanically ventilated patients demonstrated the procedure to be associated with a worsening of $\mathrm{PaO}_{2} / \mathrm{FiO}_{2}$ ratio. ${ }^{265} \mathrm{~A}$ number of case series suggest bronchoscopy and BAL are associated with variable and sometimes non-significant increases in mean arterial pressure, mean pulmonary artery pressure and $\mathrm{CO}_{2}$ in ventilated patients. $^{264}$ 266-271 Bronchoscopy and BAL are associated with worsened hypoxia and tachycardia in several studies in mechanically ventilated patients, ${ }^{239} \quad 243 \quad 263-269 \quad 271$ although these changes were clinically insignificant in most patients. In a specific study with a specific subgroup of patients with ARDS, a more than $30 \%$ drop in $\mathrm{PaO}_{2}$ was observed in $35 \%$ of patients. ${ }^{271} \mathrm{~A}$ more detailed physiological study in 18 patients undergoing bronchoscopy and BAL has demonstrated adverse change in lung compliance and resistance. ${ }^{267}$ Post-bronchoscopy pneumothorax has been reported in ventilated patients in whom transbronchial biopsy was not undertaken. ${ }^{246} 264$

\section{Evidence statements}

- Bronchoscopy with BAL in mechanically ventilated patients has been shown to be associated with a worsened oxygenation, with an $80-86 \%$ reduction in $\mathrm{PaO}_{2} / \mathrm{FiO}_{2}$ ratio from baseline regardless of the volume of BAL used. (Evidence level 1+)

- BAL has been associated with a change in lung compliance, an increase in mean arterial pressure, mean pulmonary artery pressure and rise in $\mathrm{CO}_{2}$ in ventilated patients. Hypoxia and tachycardia have been shown to be a result of BAL in several studies in mechanically ventilated patients, although these were clinically insignificant in most patients. (Evidence level 3)

- Post-bronchoscopy pneumothorax can occur in mechanically ventilated patients in the absence of TBLB. (Evidence level 3)

\section{Recommendations}

- Continuous multimodal physiological monitoring should be undertaken during and after bronchoscopy in the ICU setting. (Grade C)
- Patients should be monitored post procedure for complications, including pneumothorax even when a biopsy has not been taken. (Grade D)

\section{Ventilator settings}

Preoxygenation should be achieved by increasing the inspired oxygen concentration to $100 \%$. One hundred percent oxygen should be given during bronchoscopy and in the immediate recovery period. The ventilator should be adjusted to a mandatory setting. Triggered modes such as pressure support or assist control will not reliably maintain ventilation during bronchoscopy. The ventilator pressure limit should be increased to ensure that adequate tidal volumes are delivered during each respiratory cycle and the ventilator rate increased if necessary. Most modern microprocessor-controlled ventilators will monitor tidal volume and minute ventilation.

A special swivel connector (Portex, Hythe, UK) with a perforated diaphragm, through which the bronchoscope can be inserted, allows continued ventilation and maintenance of (positive end-expiratory pressure PEEP)/CPAP. This is particularly important when performing a bronchoscopy in patients with hypoxia (eg, ARDS) when maintenance of PEEP is important.

There is a small case series in normal human volunteers ${ }^{272}$ and a single study conducted in a dog model and six healthy human volunteers ${ }^{273}$ in support of the use of jet ventilation via the bronchoscope in ventilated patients. An 11-patient case series in healthy volunteers is in support of the use of highfrequency positive pressure ventilation during bronchoscopy. ${ }^{274}$ These techniques may be useful during bronchoscopy in the ICU, but more robust comparative studies are needed to quantify any potential benefit.

A total of three case series (totalling 46 patients) ${ }^{24} 275276$ suggest non-invasive ventilation via mask or helmet may be helpful in preventing progression to mechanical ventilation in non-ventilated patients with hypoxia undergoing bronchoscopy, but further studies are needed in this area. A small $(n=30)$ but well conducted randomised trial suggests CPAP plus oxygen is superior to oxygen alone during bronchoscopy of patients with hypoxia, preventing reduction in saturations and the need for ventilator support post procedure. ${ }^{268}$ A single case series of 40 patients assessed those requiring non-invasive ventilation for respiratory failure prior to a decision to conduct bronchoscopy. ${ }^{277}$ In these patients, $4 / 40(10 \%)$ required mechanical ventilation within $8 \mathrm{~h}$. Within $48 \mathrm{~h}, 45 \%$ were intubated, and in total 55\% required intubation (median time from bronchoscopy to intubation $=27 \mathrm{~h}$ ). This implies that patients requiring noninvasive ventilator support prior to bronchoscopy should be considered high risk for requiring intubation post procedure, and that the procedure should therefore be conducted by an experienced operator in a setting where intubation and ventilation can occur.

Monitoring intracranial pressure (ICP) in patients with a head injury is essential if sudden rises in ICP are to be avoided due to $\mathrm{CO}_{2}$ retention or other causes. Monitoring endotracheal $\mathrm{CO}_{2}$ in such patients may also help to detect falls in minute ventilation caused by the presence of the bronchoscope within the ET. Profound anaesthesia, including effective neuromuscular blockade, is often used in patients with head injury while undergoing bronchoscopy. Four studies (one randomised controlled trial ${ }^{270}$ and three others ${ }^{278-280}$ ) addressing this issue have consistently demonstrated that bronchoscopy raises ICP. A small but well conducted randomised study assessing hyperventilation via the ventilator during bronchoscopy demonstrated no difference in peak ICP, ${ }^{270}$ but suggested hyperventilation may accelerate return to baseline 
ICP after bronchoscopy in patients with closed head injury. The significance of this treatment effect on clinically important recovery is unknown. Three small non-randomised studies suggest that while ICP is significantly raised during bronchoscopy, cerebral perfusion pressure is probably maintained. ${ }^{278-280}$

Evidence statements

- A small but well conducted randomised study suggests hyperventilation via the ventilator is useful in reducing the expected rise in ICP seen during bronchoscopy in ventilated patients with closed head injury. (Evidence level 1-)

- A single but well conducted randomised trial suggests CPAP plus oxygen is superior to oxygen alone during bronchoscopy of patients with hypoxia, preventing reduction in saturations and the need for ventilator support post procedure. (Evidence level 1+)

- A total of three case series suggest non-invasive ventilation may be helpful in preventing mechanical ventilation in patients with hypoxia undergoing bronchoscopy, but further studies are needed in this area. (Evidence level 3)

\section{Recommendations}

- CPAP plus oxygen support may be considered in patients with hypoxia undergoing bronchoscopy to prevent desaturation and post-procedure requirement for mechanical ventilation. (Grade B)

- When patients require non-invasive ventilation prior to bronchoscopy, the procedure should be conducted in an environment where intubation and ventilatory support are readily accessible. (Grade D)

- Bronchoscopy should be undertaken cautiously in patients with documented or suspected raised ICP. (Grade D)

Good practice point

- Care must be exercised to ensure adequate ventilation and oxygenation is maintained during bronchoscopy in intubated patients. $(\sqrt{ })$

\section{Sedation and analgesia}

The clinical status of the patient will often determine the type and level of sedation required for bronchoscopy. Synthetic narcotics such as alfentanil or fentanyl will suppress cough and provide profound analgesia. Sedation can be induced using incremental doses of a benzodiazepine or propofol. Some patients may only require light sedation for comfort during mechanical ventilation. In such cases bronchoscopy can be undertaken with supplemental topical anaesthesia with lignocaine injected through the bronchoscope. However, care must be exercised in patients with combinations of renal failure, liver dysfunction and congestive heart failure when accumulation of lignocaine may be associated with side effects (please see section on 'Tpoical anaesthesia').

One case series of 104 patients undergoing bronchoscopy during mechanical ventilation indirectly addressed the issue of sedation level during bronchoscopy. ${ }^{271}$ The primary purpose of the study was to assess gas exchange differences before and after the procedure, however the study demonstrated significant falls in oxygenation $\left(\mathrm{PaO}_{2}<60 \mathrm{~mm} \mathrm{Hg}\right.$ with $\left.\mathrm{FiO}_{2}=0.8\right)$ in 14 patients, and this fall was independently associated in multivariate analysis with the presence of ARDS and a clinician's observation of the patient 'fighting the ventilator'. This study therefore provides weak indirect evidence that adequate sedation, to the point of the patient not fighting the ventilator, may be helpful in preventing hypoxia during bronchoscopy in ventilated patients.
Evidence statement

- Adequate sedation, to the point of the patient not fighting the ventilator, may be helpful in preventing hypoxia during FB in ventilated patients. (Evidence level 3)

Recommendations

- Adequate sedation and analgesia should be provided for patients undergoing bronchoscopy in an intensive care setting. The risks of these procedures should be carefully balanced with their potential benefit in ventilated patients. (Grade D)

- Clinicians administering sedation/anaesthesia/analgesia should be acquainted with the use of these agents, and the anaesthetist/intensivist is usually best placed to fulfil this role. (Grade D)

\section{DECONTAMINATION}

Flexible bronchoscopes are delicate, complex devices, with channels and attachments that make them difficult to clean. Although bronchoscopy carries a low risk of infection it is essential that all stages of the decontamination process are conducted correctly.

Since the last bronchoscopy guidelines were published in $2001^{2}$ the body of new evidence relating to decontamination of bronchoscopes is very limited (eight articles). Many of the recommendations are based on expert opinion.

Most bronchoscopy procedures are carried out in endoscopy units where British Society of Gastroenterology (BSG) guidelines on decontamination are followed. ${ }^{281}$ These guidelines were reviewed in $2008^{282}$; the recommendations made by BSG equally apply to bronchoscopes.

The NHS National Endoscopy Programme document 'Decontamination standards for flexible endoscopes 2008', 283 which was produced following the introduction of the Joint Advisory Group on Gastrointestinal Endoscopy accreditation, looks at safety and quality issues. This document may be considered as a basis for departmental policy. Guidelines from the Medical Devices Agency (MDA) are also available. ${ }^{284}$ A cleaning and disinfecting procedure can be found in appendix 12 of this guideline.

Many of the problems identified in the literature suggest that lack of training and poor adherence to decontamination procedures are the main cause of infection/pseudo infection. An MDA alert published in 2004 highlighted concerns with inadequate decontamination, including that of auxiliary channels. ${ }^{285}$ The Health Act 2006 stipulates the roles of decontamination leads, the need for training programmes and monitoring systems. ${ }^{286}$ Newer bronchoscopes, such as endobronchial ultrasound scopes, have extra channels, and therefore it is essential that all staff are aware of the configuration of all scopes and decontamination processes.

\section{Recommendation}

- All personnel involved in cleaning and decontaminating bronchoscopes must receive specific training in infection control practices and decontamination processes. (Grade D)

Traditionally bronchoscopes are decontaminated at the beginning of a list. When a bronchoscope has been stored in a drying cabinet or storage chamber the decontamination process prior to its use may be omitted provided it is used within the manufacturer's recommended time.

Recommendation

- Decontamination and disinfection should be carried out at the beginning and end of each list and after each patient use. 
If drying cabinets or storage chambers are unavailable bronchoscopes should be decontaminated no more than $3 \mathrm{~h}$ before the procedure to eliminate colonisation of pathogens. (Grade D)

Designated cleaning areas should ensure safe decontamination practice that allow adequate ventilation of hazardous substances and do not harm staff and patients. A one-way flow for equipment, with separate dirty and clean areas, should prevent cross contamination.

\section{Recommendation}

- Bronchoscopes should be cleaned in designated cleaning areas. Used scopes must be separated from clean scopes to prevent cross contamination. (Grade D)

All stages of the decontamination process should be carried out every time. Pre-cleaning prevents drying of particulate matter if there is unavoidable delay in the decontamination process. Standardised manual cleaning, immediately after use, removes organic material from bronchoscopes to enable thorough disinfection. All channels must always be decontaminated.

\section{Recommendation}

- Thorough cleaning, brushing and flushing of all accessible channels with enzymatic or low foaming detergent remains the most important initial stage of the cleaning process. (Grade D)

Poor cleaning and dismantling of suction valves can harbour debris and can result in continuing contamination of mycobacteria and viruses.

\section{Recommendations}

- Single-use suction valves should replace reusable valves wherever possible. Single-use valves must be discarded after each procedure. (Grade D)

- Reusable valves should be used only with one bronchoscope and stored alongside the scope for traceability. (Grade D)

The use of single-use accessories helps minimise the risk of transmission of prions.

\section{Recommendations}

- Single-use accessories should be selected over reusable accessories wherever possible. (Grade D)

- When it is necessary to use reusable accessories they must be cleaned according to manufacturer's recommendations. (Grade D)

- Tracking of patient use of equipment and cleaning processes must be completed after each use. (Grade D)

Chemical disinfectants are often toxic to the skin, mucous membranes and/or by vapour inhalation. Exposure of staff is regulated by $\mathrm{COSHH}$ guidance. Gluteraldehyde has been identified as the fifth highest cause of occupational asthma, therefore reduction of its use is recommended in favour of newer, less hazardous substances. ${ }^{287}$ There are a number of disinfectants available. The Health and Safety Executive ${ }^{288}$ offers advice on suitable alternatives.

\section{Recommendations}

- On the grounds of staff safety, manual disinfection is no longer recommended. (Grade D)

- Bronchoscopes should be processed in AERs. (Grade D)

- Aldehyde-based disinfectants are no longer recommended. (Grade C)

- Alternative, recommended disinfectants should be used in accordance with the manufacturer's instructions. (Grade D)

The choice of disinfectant will depend on compatibility with bronchoscopes and AER brand and advice should be sought from the manufacturer. Disinfectants should be used according to the manufacturer's instructions as they differ in recommended exposure time, temperature, dilution etc. The material safety data sheet must be obtained to ensure precautions are followed if applicable.

Recommendations

- Disinfectant times should be those recommended by disinfectant manufacturers. (Grade D)

- Universal decontamination procedures should be performed before and after all procedures to avoid transmission of HIV. (Grade D)

- The use of $70 \%$ alcohol after final rinse is no longer recommended as it is considered to act as a fixative. (Grade D)

Good practice points

- Compatibility of bronchoscopes with disinfectant and AER manufacturers' instructions should be checked. $(\sqrt{ })$

- A record of which bronchoscope and other reusable equipment are used on an individual patient should be kept and also of the decontamination procedure. $(\sqrt{ })$

- There is currently no known decontamination method that prevents transmission of vCJD. Record keeping and identification of high-risk cases are advised. $(\sqrt{ })$

Purpose built drying cabinets and storage chambers are now available. These devices deliver particulate filtered air through the scope channels at individual instrument appropriate temperatures and flow rates. Manufacturers claim they have been shown to prevent colonisation for up to $72 \mathrm{~h}$ or longer; their use would negate the need for bronchoscopes to undergo the decontamination cycle at the beginning of a list.

Recommendations

- Drying cabinets/storage chambers are recommended for storing clean bronchoscopes. Compatibility of bronchoscopes must be confirmed with individual instrument manufacturers. (Grade D)

- Bronchoscopes stored in drying cabinets or storage chambers should be reprocessed in accordance with the manufacturer's recommendations. (Grade D)

Bronchoscopes require storing in a manner that keeps them free from contamination from other instruments and environmental organisms. Moisture can accumulate around suction valves and ports if valves are left in situ during storage.

Recommendations

- When drying cabinets or storage chambers are not available, bronchoscopes must be stored in a hanging position, with sufficient space between instruments to avoid cross contamination. (Grade D)

- Valves must not be attached to bronchoscopes during storage. (Grade D)

- Bronchoscopes must be cleaned and disinfected before and after placing in carrying cases as these cases cannot be disinfected. Bronchoscopes should not be stored in carrying cases. (Grade D)

Mycobacteria (and subspecies), fungi, bacteria, including Pseudomonas aeruginosa and Legionella pneumophilia, have all been isolated from bronchoscope washings. Poor cleaning, storing of bronchoscopes and suction valves and contaminated tap water and AERs have all been identified as sources of contamination.

\section{Recommendation}

- A record must be kept of each bronchoscope and reusable accessory used on each individual patient. Tracking each step of the decontamination cycle and personnel involved should also be recorded. This will facilitate tracing if an increase in contamination by organisms is identified among bronchoscopy patients. (Grade D)

AER's are the recommended method of disinfecting bronchoscopes, but have been shown to be a potential source of 
infection. Validation and quality control are essential for ensuring bronchoscopes are not being contaminated with organisms during the disinfection cycle. Guidance is provided in HTM-2030 (http://www.environmentalwatersystems.co.uk/ HTM2030.htm) and from individual hospital authorised persons. The use of mains water of inappropriate quality can also be a contributory factor to the contamination of the machine and the endoscope.

\section{Recommendations}

- AERs should be self-disinfected at the beginning of each day. (Grade D)

- AERs must be validated on instillation and following introduction of new disinfectants according to HTM-01. (Grade D)

- Sterile water or filtered water should be used for the final rinse. Tap water is not recommended. (Grade D)

- Regular testing of AERs and final rinse water for mycobacteria must be carried out according to HTM-01. (Grade D)

\section{STAFF SAFETY}

Disinfectants can be hazardous to staff, they can produce irritating and sensitising vapours. Toxic effects include severe dermatitis, conjunctivitis, sinusitis and asthma.

To comply with the Health and Safety Act (1974) a detailed COSHH risk assessment must be completed and should include staff health surveillance, adequate personal protective equipment and ventilation control. A policy and equipment for dealing with spillages or disposal of disinfectants must be available. The 'Decontamination standards for flexible endoscopes 2008' document contains detailed information regarding safety issues, including staff safety. ${ }^{283}$

When handling disinfectants full personal protective equipment should be worn, including full face visors and long nitrile gloves to protect against splashes. Charcoal impregnated facemasks will protect against disinfectant vapours. Wounds and abrasions should be covered. Sealed or exhaust ventilation facilities are required to protect staff from exposure to hazardous substances such as disinfectants.

Recommendations

- Open troughs of disinfectant are not recommended. (Grade D)

- Staff handling disinfectants should always wear full personal protective equipment in line with $\mathrm{COSHH}$ risk assessment. (Grade D)

- Medical histories of staff should be recorded including preexisting asthma, skin and mucosal sensitivities. (Grade D)

- Pre-employment baseline lung function, such as spirometry, should be measured and recorded. (Grade D)

- Annual lung function measurements, such as spirometry, should be performed on all personnel directly exposed to disinfectants. (Grade D)

Bronchoscopy personnel are potentially at risk of transmission of infections such as mycobacteria. Cough-inducing procedures such as bronchoscopy pose a risk to staff from airborne mycobacteria TB. Full personal protective equipment protects staff from airborne contaminants and contaminated secretions.

There is also a potential risk to bronchoscopy personnel of blood-borne infections such as hepatitis B and HIV. Needle stick injuries can occur during re-sheathing of needles or removal of tissue from biopsy forceps. Hypodermic needles should not be used for removing tissue from forceps.

\section{Recommendations}

- Immunisation against hepatitis B and TB should be confirmed in all bronchoscopy personnel before employment. Vaccinations should be offered if necessary. (Grade D)

- Hypodermic needles or other sharp instruments should not be used to remove tissue samples from biopsy forceps. Blunt-ended needles or sterile plastic toothpicks are preferable. (Grade D)

- Reusable spiked forceps are not recommended. (Grade D)

Good practice point

- In patients with suspected TB, bronchoscopy should be performed in an appropriately engineered and ventilated area, and the bronchoscopy team should use adequate protection, including masks. $(\sqrt{ })$

\section{Nursing skill mix and staffing levels}

There is limited evidence available regarding skill mix and nurse staffing levels required during bronchoscopy procedures (www.nrls.npsa.nhs.uk/resources/? entryid45 =59896NPSA/2008/ RRR011). National Patient Safety Alert RRR011 (2008), concerning administration of sedatives during procedures such as bronchoscopy, must be considered when assessing nurse skill mix and staffing levels. The BSG guidelines $(2001)^{289}$ have made recommendations for two service models for endoscopy procedures with the recommendation that levels increase when more than one procedure room is available.

Patients require very careful monitoring of sedation levels during the procedure and throughout the recovery phase. This requires knowledge and experience of the effects and adverse effects of the sedatives and the ability to administer drugs when required.

\section{Recommendations}

- A minimum of two qualified nurses are required during bronchoscopy procedures: one assistant nurse and another dedicated to monitoring patients response to the medication and procedure. (Grade D)

- A qualified nurse is required to recover a patient after bronchoscopy. (Grade D)

- Advanced procedures may require additional staff. (Grade D) Numbers of ancillary staff, for support and cleaning of equipment, may vary according to location and cleaning methods of bronchoscopes.

\section{PATIENT SATISFACTION}

FB is necessary for the diagnosis and endobronchial treatment of respiratory disease. Many patients are fearful of the procedure and experience anxiety prior to and discomfort during the bronchoscopy, irrespective of sedation and analgesia. ${ }^{290} 291$ Increasingly emphasis is placed on patient satisfaction as an outcome measure for bronchoscopy, along with the diagnostic and therapeutic value of the procedure. ${ }^{292}$

Patient satisfaction is complex and influenced by multiple factors, measured in varied ways, and needs to be interpreted in the context of the specific procedure evaluated. Multiple factors appear to influence patient satisfaction when assessed for FB. Patient characteristics, previous healthcare experience, expectations, the procedure and post-procedure care all play a role in determining how satisfied a patient is following a FB. Patient's willingness to return for another bronchoscopy, ${ }^{292}$ visual analogue scoring (VAS) systems, adherence to post-procedure instructions and recorded numbers of complaints and medical 
negligence claims are mentioned as indicators of satisfaction in bronchoscopy.

Determining factors for patient satisfaction with bronchoscopy can be divided into patient characteristics, process or procedure factors and factors associated with the setting. Lechtzin et $a l^{292}$ found that patients more willing to return for a repeat bronchoscopy had a better general health status, experienced less pain with bronchoscope insertion, rated the information given to them better and rated the quality of the bronchoscopist higher. Hirose et al ${ }^{293}$ found that male gender, shorter examination time, more experienced bronchoscopist and less discomfort from coughing, pharyngeal pain and swallowing were associated with higher patient satisfaction. VAS for cough perception during a FB appear to correlate best with patient satisfaction in one study. ${ }^{294}$ This study further demonstrates a positive correlation between VAS for satisfaction as reported by the bronchoscopist and the patient and the total number of coughs recorded during the procedure. Bernasconi et al reported the length of the procedure as an important determining factor for satisfaction, irrespective of procedures performed during the bronchoscopy. ${ }^{295}$

Many patients undergoing a bronchoscopy are fearful of a malignant diagnosis and are anxious prior to the procedure. The discomfort experienced by patients during bronchoscopy strongly correlates to anxiety before the procedure. ${ }^{295} 296$ Adequate sedation reduces patient anxiety and improves tolerance and satisfaction with the procedure. ${ }^{98}$ Detailed preprocedure information explaining 'how' the procedure will be done and 'why' it is indicated helps to reduce pre-procedure fear in patients ${ }^{290}$ (appendix 13 provides an example of a patient information leaflet that is also available electronically on the BTS website).

Distraction methods have been used to reduce patients' levels of fear and anxiety prior to bronchoscopy. Distraction therapy with nature sounds and sights improved self-reported anxiety in a randomised trial done by Diette et al. ${ }^{297}$ Two randomised trials using music to reduce stress in patients failed to demonstrate a difference in physiological responses during the procedure $^{298}$ or procedure-related anxiety state scores. ${ }^{299}$

There is insufficient evidence to support a specific time for patients to starve prior to bronchoscopy. A Cochrane review and recent systematic review on fasting times for surgery found no evidence that participants given fluids $2-3 \mathrm{~h}$ preoperatively were at an increased risk of aspiration/regurgitation. ${ }^{300} 301$ The 2001 guideline recommended fasting time for solids of $4 \mathrm{~h}$ and clear fluids up to $2 \mathrm{~h}^{2}$ No new evidence was found of increased adverse events with this regime or evidence to support longer fasting times for FB.

Patient satisfaction is complex and influenced by multiple factors; it is increasingly recognised that measuring the patient experience in a specific setting is important to improve and transform services. The Department of Health in the UK published a document on best practice in 2009 entitled 'Understanding what matters: a guide to using patient feedback to transform care'. ${ }^{302}$ In this document the importance of patient involvement in developing and changing healthcare services is emphasised. The process of collecting and reviewing patient experience feedback is important in how healthcare services are delivered. ${ }^{302}$ The benefits of including patient feedback in review of FB services include:

- improvement in communication between patients and staff;

- building trust and confidence in the healthcare provider and service;
- informs planning and service improvement;

- patient-centred, accessible and responsive services;

- patients help to shape the services that they use.

Evidence statements

- Patient satisfaction is determined by multiple factors and depends on patient specific factors, the procedure, the setting, information given prior to the procedure, sedation used and post-procedure management. (Evidence level 3)

- Verbal and written information given prior to FB, explaining the indications and what the patient can expect during the procedure, improve patient tolerance and satisfaction. (Evidence level 3)

- Patients offered sedation during FB tolerate the procedure better and are less likely to have a failed procedure. (Evidence level 2)

Recommendations

- Verbal and written patient information explaining indications and what to expect during the procedure, and potential complications should be provided to improve patient tolerance. (Grade C)

- Patients should be offered sedation during FB to improve patient tolerance. (Grade B)

- It is sufficient for patients to have no food by mouth for $4 \mathrm{~h}$ and to allow clear fluids by mouth up to $2 \mathrm{~h}$ before bronchoscopy. (Grade D)

Good practice point

- Patients who had sedation should be advised not to drive, sign legally binding documents or operate machinery for $24 \mathrm{~h}$ after the procedure $(\sqrt{ })$

\section{CONSENT}

Patients have a right to information about their condition and the treatment options open to them and practitioners should be satisfied that they have consent from a patient, or other valid authority, before undertaking FB. Giving and obtaining consent is usually a process, not a one-off event. Patients need time to read and understand the information provided before FB and should be given the opportunity to ask questions. Patients can change their minds and withdraw consent at any time. To proceed with bronchoscopy in the absence of appropriate consent may lead to legal proceedings for assault and battery. Failure to provide adequate information to a patient, including information about the potential risks and adverse outcomes of a procedure, may lead to a negligence action against the clinician. In the UK, the principles and guidance for obtaining informed consent is outlined by the General Medical Council in a document entitled 'Consent guidance: patients and doctors making decisions together'. ${ }^{303}$

\section{Good practice point}

- Practitioners undertaking FB should be familiar with, and adhere to the national and local guidance for obtaining informed consent. $(\sqrt{ })$

The practitioner undertaking the bronchoscopy or responsible for the trainee doing the bronchoscopy is responsible for ensuring valid consent was obtained prior to the procedure. If it is not practical for this clinician to discuss the procedure with the patient, the responsibility can be delegated to someone else, provided the person the task is delegated to:

- has been suitably trained and is qualified;

- has sufficient knowledge of the proposed procedure and/or treatment to understand and discuss the risks involved with the patient; 
- has a good understanding, and agrees to act in accordance with national and local guidance on obtaining informed consent.

The patient should be provided with timely and sufficient information about the proposed FB before the procedure date. The exchange of information between the clinician and the patient is central to the process of informed consent and good decision-making. The amount of information shared with patients will vary, depending on their individual circumstances. The approach to discussions with patients should be tailored to:

- the patient's individual needs, wishes and priorities;

- the patient's unique level of understanding and knowledge of their condition, prognosis and the treatment options;

- the nature of the condition that the bronchoscopy is indicated for;

- the nature and level of risk associated with the proposed procedure.

Patients should be given the opportunity to ask any questions and practitioners must answer all questions honesty and truthfully. Care should be taken not to make any assumptions about the amount of information the patient may need or want or the patient's knowledge or understanding of the proposed procedure. A suggested patient information leaflet accompanies this guideline in appendix 13.

\section{CONCLUSION}

$\mathrm{FB}$ is an essential, established and ever expanding tool in respiratory medicine. Its practice, however, needs to be safe, effective and for the right indications.

This guideline seeks to provide a detailed, evidence-based and practical overview of best practice in this field. Extensive literature has been published in the various aspects of bronchoscopy over the last three decades, and this document has been developed by carefully appraising all the available publications, applying a rigorous, standardised methodology, and summarising the wealth of data that are available.

Particular emphasis has been placed on the safety aspects of bronchoscopy, such as patient monitoring, precautions in specific conditions, prevention and management of complications, adequate staffing, optimal sedation and disinfection. At the same time, the importance of continually auditing the practice of this indispensable technique, with the aim of achieving and maintaining the high diagnostic utility that it offers is outlined.

The primary objective of this guideline is to provide practitioners in the UK and beyond with a comprehensive guide to the technique of basic FB, using up-to-date information. For information on more advanced bronchoscopic procedures, this document is best read in conjunction with the BTS guideline for advanced diagnostic and therapeutic bronchoscopy, published in November $2011 .^{3}$

\section{REFERENCES}

1 Ikeda S, Yanai N, Ishikawa S. Flexible bronchofiberscope. Keio J Med 1968:17:1-16.

2 British Thoracic Society Bronchoscopy Guidelines Committee. British Thoracic Society guidelines on diagnostic flexible bronchoscopy. Thorax 2001;56(Suppl 1): i1-21.

3 Du Rand IA, Barber PV, Goldring J, et al. British Thoracic Society guideline for advanced diagnostic and therapeutic flexible bronchoscopy in adults. Thorax 2011;66(Suppl 3):iii1-iii21.

4 Facciolongo N, Patelli M, Gasparini S, et al. Incidence of complications in bronchoscopy. Multicentre prospective study of 20,986 bronchoscopies. Monaldi Arch Chest Dis 2009;71:8-14.
5 Hehn BT, Haponik E, Rubin HR, et al. The relationship between age and process of care and patient tolerance of bronchoscopy. J Am Geriatr SoC 2003;51:917-22.

6 Bechara R, Beamis J, Simoff $M$, et al. Practice and complications of flexible bronchoscopy with biopsy procedures. J Bronchol 2005;12:139-42.

7 Hehn B, Haponik EF. Flexible bronchoscopy in the elderly. Clin Chest Med 2001:22:301-9.

8 Van Zwam JP, Kapteijns EFG, Lahey S, et al. Flexible bronchoscopy in supine or sitting position: a randomized prospective analysis of safety and patient comfort. J Bronchol 2010;17:29-32.

9 Yildiz P, Ozgul A, Yimaz V. Changes in oxygen saturation in patients undergoing fiberoptic bronchoscopy. Chest 2002;121:1007-8.

10 Maranetra N, Pushpakom R, Bovornkitti S. Oxygen desaturation during fibreoptic bronchoscopy. J Med Assoc Thai 1990;73:258-63.

11 Milman N, Faurschou P, Grode G, et al. Pulse oximetry during fibreoptic bronchoscopy in local anaesthesia: frequency of hypoxaemia and effect of oxygen supplementation. Respiration, 1994;61:342-7.

12 Attaran D, Towhidi M, Amini M, et al. The relationship between peak expiratory flow rate before bronchoscopy and arterial oxygen desaturation during bronchoscopy. Acta Medica Iranica 2008;46:95-8.

13 Lin CC, Wu JL, Huang WC. Pulmonary function in normal subjects after bronchoalveolar lavage. Chest 1988;93:1049-53.

14 Markou NK, Kanakaki MC, Boutzouka E, et al. Fluctuations in gas exchange and cardiovascular parameters during flexible bronchoscopy. J Bronchol 1999;6:241-6.

15 Van Vyve T, Chanez P, Bousquet J, et al. Safety of bronchoalveolar lavage and bronchial biopsies in patients with asthma of variable severity. Am Rev Respir Dis 1992;146:116-21.

16 Meghjee SP, Marshall M, Redfern EJ, et al. Influence of patient posture on oxygen saturation during fibre-optic bronchoscopy. Respir Med 2001;95:5-8.

17 Arai T, Hatano Y, Komatsu K, et al. Real-time analysis of the change in arterial oxygen tension during endotracheal suction with a fiberoptic bronchoscope. Crit Care Med 1985;13:855-8.

18 Lundgren R, Haggmark S, Reiz S. Hemodynamic effects of flexible fiberoptic bronchoscopy performed under topical anesthesia. Chest 1982;82:295-9.

19 Fang W-F, Chen Y-C, Chung Y-H, et al. Predictors of oxygen desaturation in patients undergoing diagnostic bronchoscopy. Chang Gung Med J 2006;29:306-12.

20 Payne CB Jr, Goyal PC, Gupta SC. Effects of transoral and transnasal fiberoptic bronchoscopy on oxygenation and cardiac rhythm. Endoscopy 1986;18:1-3.

21 Jones AM, O'Driscoll R. Do all patients require supplemental oxygen during flexible bronchoscopy? Chest 2001;119:1906-9.

22 Schiffman PL, Westlake RE, Fourre JA, et al. Arterial oxygen saturation and cardiac rhythm during transoral fiberoptic bronchoscopy. J Med Soc N J 1982;79:723-6.

23 Pirozynski M, Sliwinski P, Zielinski J. Effect of different volumes of BAL fluid on arterial oxygen saturation. Eur Respir J 1988;1:943-7.

24 Antonelli M, Pennisi MA, Conti G, et al. Fiberoptic bronchoscopy during noninvasive positive pressure ventilation delivered by helmet. Intensive Care Med 2003;29:126-9.

25 Cole $\mathrm{P}$, Turton $\mathrm{C}$, Lanyon $\mathrm{H}$, et al. Bronchoalveolar lavage for the preparation of free lung cells: technique and complications. Br J Dis Chest 1980;74:273-8.

26 Sharma GD, Bansal SK, Kashyap S, et al. Effect of fiberoptic bronchoscopy on arterial blood gases and cardiac rhythm at a moderate altitude of 2250 meters. J Assoc Physicians India 1999:47:1056-9.

27 Hendy MS, Bateman JR, Stableforth DE. The influence of transbronchial lung biopsy and bronchoalveolar lavage on arterial blood gas changes occurring in patients with diffuse interstitial lung disease. Br J Dis Chest 1984;78:363-8.

28 Ouellette DR, Diaz J. Elevation of the double product during flexible bronchoscopy: effects of uncontrolled hypertension and the use of beta-blockade. J Bronchol 2008;15:73-7.

29 Davies L, Mister R, Spence DP, et al. Cardiovascular consequences of fibreoptic bronchoscopy. Eur Respir J 1997;10:695-8.

30 Katz AS, Michelson EL, Stawicki J, et al. Cardiac arrhythmias. Frequency during fiberoptic bronchoscopy and correlation with hypoxemia. Arch Intern Med 1981;141:603-6.

31 Dweik RA, Mehta AC, Meeker DP, et al. Analysis of the safety of bronchoscopy after recent acute myocardial infarction. Chest 1996;110:825-8.

32 Ernst A, Eberhardt R, Wahidi $M$, et al. Effect of routine clopidogrel use on bleeding complications after transbronchial biopsy in humans. Chest 2006:129:734-7.

33 Kozak EA, Brath LK. Do 'screening' coagulation tests predict bleeding in patients undergoing fiberoptic bronchoscopy with biopsy? Chest 1994;106:703-5.

34 Cordasco EM Jr, Mehta AC, Ahmad M. Bronchoscopically induced bleeding. A summary of nine years' Cleveland clinic experience and review of the literature. Chest 1991;100:1141-7.

35 Bjortuff O, Brosstad F, Boe J. Bronchoscopy with transbronchial biopsies: measurement of bleeding volume and evaluation of the predictive value of coagulation tests. Eur Respir J 1998;12:1025-7. 
36 Diette GB, Wiener CM, White P Jr. The higher risk of bleeding in lung transplant recipients from bronchoscopy is independent of traditional bleeding risks: results of a prospective cohort study. Chest 1999;115:397-402.

37 Weiss SM, Hert RC, Gianola FJ, et al. Complications of fiberoptic bronchoscopy in thrombocytopenic patients. Chest 1993;104:1025-8.

38 Mehta NL, Harkin TJ, Rom WN, et al. Should renal insufficiency be a relative contraindication to bronchoscopic biopsy? J Bronchol 2005:12:81-3.

39 Pue CA, Pacht ER. Complications of fiberoptic bronchoscopy at a university hospital. Chest 1995;107:430-2.

40 Prickett C, LeGrand P. Complications of fiberoptic bronchoscopy in a community hospital. Ala Med 1984;53:25-7.

41 D'Ippolito R, Foresi A, Castagnetti C, et al. Indications for flexible fiberoptic bronchoscopy and its safety in the very elderly. Monaldi Arch Chest Dis 2007;67:23-9.

42 Colt HG, Matsuo T. Hospital charges attributable to bronchoscopy-related complications in outpatients. Respiration 2001:68:67-72.

43 Milman N, Faurschou P, Munch EP, et al. Transbronchial lung biopsy through the fibre optic bronchoscope. Results and complications in 452 examinations. Respir Med 1994;88:749-53.

44 Izbicki G, Shitrit D, Yarmolovsky A, et al. Is routine chest radiography after transbronchial biopsy necessary? A prospective study of 350 cases. Chest 2006;129:1561-4

45 Ibrahim AS, Allangawi MH, Sattar HA, et al. Indications, diagnostic yields and complications of transbronchial biopsy over 5 years in the State of Qatar. Saudi Med J 2005:26:641-5.

46 Hue SH. Complications in transbronchial lung biopsy. Korean J Intern Med 1987:2:209-13.

47 Ahmad M, Livingston DR, Golish JA, et al. The safety of outpatient transbronchial biopsy. Chest 1986;90:403-5.

48 Milam MG, Evins AE, Sahn SA. Immediate chest roentgenography following fiberoptic bronchoscopy. Chest 1989;96:477-9.

49 Sharif-Kashani B, Shahabi P, Behzadnia N, et al. Incidence of fever and bacteriemia following flexible fiberoptic bronchoscopy: a prospective study. Acta Medica Iranica 2010:48:385-8.

50 Um S-W, Choi C-M, Lee C-T, et al. Prospective analysis of clinical characteristics and risk factors of postbronchoscopy fever. Chest 2004;125:945-52.

51 Huang JCT, Bassett MA, Levin D, et al. Acute phase reaction in healthy volunteers after bronchoscopy with lavage. Chest 2006;129:1565-9.

52 Georgiades G, Myrianthefs P, Venetsanou K, et al. Temperature and serum proinflammatory cytokine changes in patients with NSCLC after BAL. Lung 2003; 181:35-47.

53 Park JS, Lee $\mathrm{CH}$, Yim JJ, et al. Impact of antibiotic prophylaxis on postbronchoscopy fever: a randomised controlled study. Int J Tuberc Lung Dis 2011;15:528-35.

54 Haynes J, Greenstone MA. Fibreoptic bronchoscopy and the use of antibiotic prophylaxis. Br Med J Clin Res ed 1987;294:1199.

55 Yigla M, Oren I, Bentur L, et al. Incidence of bacteraemia following fibreoptic bronchoscopy. Eur Respir J 1999;14:789-91.

56 Centre for Clinical Practice at NICE. Prophylaxis against infective endocarditis (CG64). London: National Institute for Health and Clinical Excellence (NICE), 2008.

57 Djukanovic R, Wilson JW, Lai CK, et al. The safety aspects of fiberoptic bronchoscopy, bronchoalveolar lavage, and endobronchial biopsy in asthma. Am Rev Respir Dis 1991:143(4 Pt 1):772-7.

58 Moore WC, Evans MD, Bleecker ER, et al. Safety of investigative bronchoscopy in the Severe Asthma Research Program. J Allergy Clin Immunol 2011;128:328-36.e3.

59 Kariyawasam HH, Aizen M, Kay AB, et al. Safety and tolerability of three consecutive bronchoscopies after allergen challenge in volunteers with mild asthma. Thorax 2007;62:557-8.

60 Rankin JA, Snyder PE, Schachter EN, et al. Bronchoalveolar lavage. Its safety in subjects with mild asthma. Chest 1984:85:723-8.

61 Humbert M, Robinson DS, Assoufi B, et al. Safety of fibreoptic bronchoscopy in asthmatic and control subjects and effect on asthma control over two weeks. Thorax 1996;51:664-9.

62 Elston WJ, Whittaker AJ, Khan LN, et al. Safety of research bronchoscopy, biopsy and bronchoalveolar lavage in asthma. Eur Respir J 2004;24:375-7.

63 Tapanainen L, Lindqvist A, Halme M, et al. Investigative bronchoscopy and endobronchial biopsy is well tolerated in hyperreactive asthma patients. Respir Med 2002;96:466-8.

64 Peacock AJ, Benson-Mitchell R, Godfrey R. Effect of fibreoptic bronchoscopy on pulmonary function. Thorax 1990;45:38-41.

65 Chechani V. Flexible bronchoscopy in patients with hypercapnia. J Bronchol 2000;7:226-32

66 Stolz D, Pollak V, Chhajed PN, et al. A randomized, placebo-controlled trial of bronchodilators for bronchoscopy in patients with COPD. Chest 2007;131:765-72.

67 Hattotuwa KL, Gizycki MJ, Ansari TW, et al. The effects of inhaled fluticasone on airway inflammation in chronic obstructive pulmonary disease: a double-blind, placebo-controlled biopsy study. Am J Respir Crit Care Med 2002;165:1592-6.
68 Dunagan DP, Burke HL, Aquino SL, et al. Fiberoptic bronchoscopy in coronary care unit patients: indications, safety, and clinical implications. Chest 1998;114:1660-7.

69 Fleisher LA, Beckman JA, Brown KA, et al. ACC/AHA 2007 guidelines on perioperative cardiovascular evaluation and care for noncardiac surgery. Circulation 2007;116:e418-500

70 Ozgul MA, Turna A, Yildiz P, et al. Risk factors and recurrence patterns in 203 patients with hemoptysis. Tuberk Toraks 2006;54:243-8.

71 Thirumaran $M$, Sundar $R$, Sutcliffe IM, et al. Is investigation of patients with haemoptysis and normal chest radiograph justified? Thorax 2009;64:854-6.

72 O'Hickey S, Hilton AM. Fibreoptic bronchoscopy in the elderly. Age Ageing 1987:16:229-33.

73 Macfarlane JT, Storr A, Wart MJ, et al. Safety, usefulness and acceptability of fibreoptic bronchoscopy in the elderly. Age Ageing 1981;10:127-31.

74 Knox AJ, Mascie-Taylor BH, Page RL. Fibreoptic bronchoscopy in the elderly: 4 years' experience. Br J Dis Chest 1988;82:290-3.

75 Rokach A, Fridlender ZG, Arish N, et al. Bronchoscopy in octogenarians. Age Ageing 2008;37:710-13.

76 Glazer M, Breuer R, Berkman N, et al. Use of fiberoptic bronchoscopy in bone marrow transplant recipients. Acta Haematol 1998;99:22-6.

77 Azoulay E, Mokart D, Rabbat A, et al. Diagnostic bronchoscopy in hematology and oncology patients with acute respiratory failure: prospective multicenter data. Crit Care Med 2008:36:100-7.

78 Dunagan DP, Baker AM, Hurd DD, et al. Bronchoscopic evaluation of pulmonary infiltrates following bone marrow transplantation. Chest 1997:111:135-41.

79 Hofmeister CC, Czerlanis C, Forsythe S, et al. Retrospective utility of bronchoscopy after hematopoietic stem cell transplant. Bone Marrow Transplant 2006;38:693-8

80 Feinstein MB, Mokhtari M, Ferreiro R, et al. Fiberoptic bronchoscopy in allogeneic bone marrow transplantation: findings in the era of serum cytomegalovirus antigen surveillance. Chest 2001:120:1094-100.

81 Jain $P$, Sandur $S$, Meli $Y$, et al. Role of flexible bronchoscopy in immunocompromised patients with lung infiltrates. Chest 2004;125:712-22.

82 Shannon VR, Andersson BS, Lei $X$, et al. Utility of early versus late fiberoptic bronchoscopy in the evaluation of new pulmonary infiltrates following hematopoietic stem cell transplantation. Bone Marrow Transplant 2010;45:647-55.

83 Chhajed PN, Glanville AR. Management of hypoxemia during flexible bronchoscopy. Clin Chest Med 2003;24:511-16.

84 McWilliams TJ, Williams TJ, Whitford HM, et al. Surveillance bronchoscopy in lung transplant recipients: risk versus benefit. J Heart Lung Transplant 2008;27:1203-9.

85 Smith $\mathrm{L}$, Singer JP, Hayes $\mathrm{M}$, et al. An analysis of potential risk factors for early complications from fiberoptic bronchoscopy in lung transplant recipients. Transp/ Int 2012;25:172-8.

86 Hopkins PM, Aboyoun CL, Chhajed PN, et al. Prospective analysis of 1,235 transbronchial lung biopsies in lung transplant recipients. J Heart Lung Transplant 2002;21:1062-7

87 Malik JA, Gupta D, Agarwal AN, et al. Anticholinergic premedication for flexible bronchoscopy: a randomized, double-blind, placebo-controlled study of atropine and glycopyrrolate. Chest 2009;136:347-54.

88 Williams T, Brooks T, Ward C. The role of atropine premedication in fiberoptic bronchoscopy using intravenous midazolam sedation. Chest 1998;113:1394-8.

89 Cowl CT, Prakash UB, Kruger BR. The role of anticholinergics in bronchoscopy. A randomized clinical trial. Chest 2000;118:188-92.

90 Matot I, Kramer MR. Sedation in outpatient bronchoscopy. Respir Med 2000;94:1145-53.

91 de Padua Al, de Castro M, Schmidt A, et al. Clonidine as a pre-anesthetic agent for flexible bronchoscopy. Respir Med 2004;98:746-51.

92 Fox BD, Krylov $Y$, Leon $\mathrm{P}$, et al. Benzodiazepine and opioid sedation attenuate the sympathetic response to fiberoptic bronchoscopy. Prophylactic labetalol gave no additional benefit. Results of a randomized double-blind placebo-controlled study. Respir Med 2008:102:978-83.

93 Vesco D, Kleisbauer JP, Orehek J. Attenuation of bronchofiberscopy-induced cough by an inhaled beta 2-adrenergic agonist, fenoterol. Am Rev Respir Dis 1988;138:805-6.

94 Schwarz Y, Greif J, Lurie 0, et al. Dextromethorphan premedication reduces midazolam requirement: objective and subjective parameters in peribronchoscopy. Respiration 2007:74:314-19.

95 Maltais F, Laberge F, Laviolette M. A randomized, double-blind, placebo-controlled study of lorazepam as premedication for bronchoscopy. Chest 1996;109:1195-8.

96 Pickles J, Jeffrey M, Datta A, et al. Is preparation for bronchoscopy optimal? Eur Respir J 2003;22:203-6.

97 Smyth CM, Stead RJ. Survey of flexible fibreoptic bronchoscopy in the United Kingdom. Eur Respir J 2002;19:458-63.

98 Putinati S, Ballerin L, Corbetta L, et al. Patient satisfaction with conscious sedation for bronchoscopy. Chest 1999;115:1437-40.

99 Gonzalez R, De-La-Rosa-Ramirez I, Maldonado-Hernandez A, et al. Should patients undergoing a bronchoscopy be sedated? Acta Anaesthesio/ Scand 2003:47:411-15. 
100 Hatton MQ, Allen MB, Vathenen AS, et al. Does sedation help in fibreoptic bronchoscopy? BMJ 1994;309:1206-7.

101 Silvestri GA, Vincent BD, Wahidi MM, et al. A phase 3, randomized, double-blind study to assess the efficacy and safety of fospropofol disodium injection for moderate sedation in patients undergoing flexible bronchoscopy. Chest 2009;135:41-7

102 Maguire GP, Rubinfeld AR, Trembath PW, et al. Patients prefer sedation for fibreoptic bronchoscopy. Respirology 1998;3:81-5.

103 De $S$. Assessment of patient satisfaction and lidocaine requirement during flexible bronchoscopy without sedation. J Bronchol 2009;16:176-9.

104 Morris LG, Zeitler DM, Amin MR. Unsedated flexible fiberoptic bronchoscopy in the resident clinic: technique and patient satisfaction. Laryngoscope 2007;117:1159-62

105 Reducing risk of overdose with midazolam injection in adults. NPSA/2008/ RRR011. 2008. http://www.nrls.npsa.nhs.uk/resources/?entryid45=59896 (accessed Jun 2013).

106 The Royal College of Anaesthestists. Implementing and ensuring safe sedation practice for healthcare procedures in adults. UK Academy of Medical Royal Colleges and their Faculties. 2001. http://www.rcoa.ac.uk/node/2270 (accessed Jun 2013).

107 Ramsay MA, Savege TM, Simpson BR, et al. Controlled sedation with alphaxalone-alphadolone. Br Med J 1974;2:656-9.

108 Chernik DA, Gillings D, Laine $H$, et al. Validity and reliability of the Observer's Assessment of Alertness/Sedation Scale: study with intravenous midazolam. J Clin Psychopharmacol 1990;10:244-51.

109 Clarkson K, Power CK, O'Connell F, et al. A comparative evaluation of propofol and midazolam as sedative agents in fiberoptic bronchoscopy. Chest 1993;104:1029-31.

110 Clark G, Licker M, Younossian AB, et al. Titrated sedation with propofol or midazolam for flexible bronchoscopy: a randomised trial. Eur Respir J 2009;34:1277-83.

111 Crawford M, Pollock J, Anderson K, et al. Comparison of midazolam with propofo for sedation in outpatient bronchoscopy. Br J Anaesth 1993;70:419-22.

112 Greig JH, Cooper SM, Kasimbazi HJ, et al. Sedation for fibre optic bronchoscopy. Respir Med 1995;89:53-6.

113 Houghton CM, Raghuram A, Sullivan PJ, et al. Pre-medication for bronchoscopy: a randomised double blind trial comparing alfentanil with midazolam. Respir Med 2004;98:1102-7.

114 Hwang J, Jeon Y, Park HP, et al. Comparison of alfetanil and ketamine in combination with propofol for patient-controlled sedation during fiberoptic bronchoscopy. Acta Anaesthesio/ Scand 2005:49:1334-8.

115 Lo YL, Lin TY, Fang YF, et al. Feasibility of bispectral index-guided propofol infusion for flexible bronchoscopy sedation: a randomized controlled trial. PLOS ONE 2011;6:e27769.

116 Schlatter L, Pflimlin E, Fehrke B, et al. Propofol versus propofol plus hydrocodone for flexible bronchoscopy: a randomised study. Eur Respir J 2011;38:529-37.

117 Stolz D, Chhajed PN, Leuppi JD, et al. Cough suppression during flexible bronchoscopy using combined sedation with midazolam and hydrocodone: a randomised, double blind, placebo controlled trial. Thorax 2004:59:773-6.

118 Stolz D, Kurer G, Meyer A, et al. Propofol versus combined sedation in flexible bronchoscopy: a randomised non-inferiority trial. Eur Respir J 2009;34:1024-30.

119 Dundee JW, Collier PS, Carlisle RJ, et al. Prolonged midazolam elimination half-life. Br J Clin Pharmacol 1986;21:425-9.

120 Dreher $\mathrm{M}$, Ekkernkamp $\mathrm{E}$, Storre $\mathrm{JH}$, et al. Sedation during flexible bronchoscopy in patients with pre-existing respiratory failure: midazolam versus midazolam plus alfentanil. Respiration 2010;79:307-14.

121 Korttila K, Saarnivaara L, Tarkkanen J, et al. Effect of age on amnesia and sedation induced by flunitrazepam during local anaesthesia for bronchoscopy. $\mathrm{Br} J$ Anaesth 1978;50:1211-18.

122 Gray AJG, Bell GD. Elderly patients vulnerable because of excessive doses of sedatives. National Confidential Enquiry into Patient Outcome and Death (NCEPOD). http://www.ncepod.org.uk/pdf/current/NPSA\%20sedation\%20article.pdf (accessed Jun 2013)

123 Cullinane M, Gray AJG, Hargraves CMK, et al. Scoping our practice. The 2004 Report of the National Confidential Enquiry into Patient Outcome and Death. 2004.

124 Diaz-Fuentes G, Dalvi A, Blum S, et al. Requirement of sedation during flexible bronchoscopy among substance and nonsubstance users. J Bronchol 2006;13:58-60.

125 Antoniades N, Worsnop C. Topical lidocaine through the bronchoscope reduces cough rate during bronchoscopy. Respirology 2009;14:873-6.

126 Guay J. Methemoglobinemia related to local anesthetics: a summary of 242 episodes. Anesth Analg 2009;108:837-45.

127 Osula S, Stockton P, Abdelaziz MM, et al. Intratracheal cocaine induced myocardial infarction: an unusual complication of fibreoptic bronchoscopy. Thorax 2003:58:733-4

128 Benzaquen BS, Cohen V, Eisenberg MJ. Effects of cocaine on the coronary arteries Am Heart J 2001;142:402-10.
129 Randell T, Yli-Hankala A, Valli $\mathrm{H}$, et al. Topical anaesthesia of the nasal mucosa for fibreoptic airway endoscopy. Br J Anaesth 1992;68:164-7.

130 Webb AR, Woodhead MA, Dalton HR, et al. Topical nasal anaesthesia for fibreoptic bronchoscopy: patients' preference for lignocaine gel. Thorax 1989:44:674-5

131 Zainudin BM, Rafia MH, Sufarlan AW. Topical nasal anaesthesia for fibreoptic bronchoscopy: lignocaine spray or gel? Singapore Med J 1993;34:148-9.

132 Keane D, McNicholas WT. Comparison of nebulized and sprayed topical anaesthesia for fibreoptic bronchoscopy. Eur Respir J 1992;5:1123-5.

133 Webb J. Local anaesthesia for fibreoptic bronchoscopy - where are we now? Respir Med 1990;84:349-50.

134 Isaac PA, Barry JE, Vaughan RS, et al. A jet nebuliser for delivery of topical anesthesia to the respiratory tract. A comparison with cricothyroid puncture and direct spraying for fibreoptic bronchoscopy. Anaesthesia 1990;45:46-8.

135 Stolz D, Chhajed PN, Leuppi J, et al. Nebulized lidocaine for flexible bronchoscopy: a randomized, double-blind, placebo-controlled trial. Chest 2005;128:1756-60.

136 Hasmoni MH, Rani MFA, Harun R, et al. Randomized-controlled trial to study the equivalence of $1 \%$ versus $2 \%$ lignocaine in cough suppression and satisfaction during bronchoscopy. J Bronchol Interv Pulmonol 2008;15:78-82.

137 Mainland PA, Kong AS, Chung DC, et al. Absorption of lidocaine during aspiration anesthesia of the airway. J Clin Anesth 2001:13:440-6.

138 Xue FS, Liu HP, He N, et al. Spray-as-you-go airway topical anesthesia in patients with a difficult airway: a randomized, double-blind comparison of $2 \%$ and $4 \%$ lidocaine. Anesth Analg 2009;108:536-43.

139 Efthimiou J, Higenbottam T, Holt D, et al. Plasma concentrations of lignocaine during fibreoptic bronchoscopy. Thorax 1982;37:68-71.

140 Milman N, Laub M, Munch EP, et al. Serum concentrations of lignocaine and its metabolite monoethylglycinexylidide during fibre-optic bronchoscopy in local anaesthesia. Respir Med 1998;92:40-3.

141 Frey WC, Emmons EE, Morris MJ. Safety of high dose lidocaine in flexible bronchoscopy. J Bronchol 2008;15:33-7.

142 Martin KM, Larsen PD, Segal R, et al. Effective nonanatomical endoscopy training produces clinical airway endoscopy proficiency. Anesth Analg 2004:99:938-44.

143 Langmack EL, Martin RJ, Pak J, et al. Serum lidocaine concentrations in asthmatics undergoing research bronchoscopy. Chest 2000;117:1055-60.

144 Ameer B, Burlingame MB, Harman EM. Systemic absorption of topical lidocaine in elderly and young adults undergoing bronchoscopy. Pharmacotherapy 1989;9:74-81.

145 Rivera MP, Mehta AC. Initial diagnosis of lung cancer. Chest 2007;132(3 Suppl):131S-48S.

146 McLean AN, Semple PA, Franklin DH, et al. The Scottish multi-centre prospective study of bronchoscopy for bronchial carcinoma and suggested audit standards. Respir Med 1998:92:1110-15.

147 Gellert AR, Rudd RM, Sinha G, et al. Fibreoptic bronchoscopy: effect of multiple bronchial biopsies on diagnostic yield in bronchial carcinoma. Thorax 1982;37:684-7.

148 Popovich J Jr, Kvale PA, Eichenhorn MS, et al. Diagnostic accuracy of multiple biopsies from flexible fiberoptic bronchoscopy. A comparison of central versus peripheral carcinoma. Am Rev Respir Dis 1982;125:521-3.

149 Slade MG, Rahman NM, Stanton AE, et al. Improving standards in flexible bronchoscopy for lung cancer. Eur Respir J 2011;37:895-901.

150 Hetzel J, Eberhardt R, Herth FJF, et al. Cryobiopsy increases the diagnostic yield of endobronchial biopsy: a multicentre trial. Eur Respir J 2012;39:685-90.

151 Navani N, Brown JM, Nankivell $M$, et al. Suitability of EBUS-TBNA specimens for subtyping and genotyping of NSCLC: a multi-centre study of 774 patients. Am J Respir Crit Care Med 2012;185:1316-22.

152 van der Drift MA, van der Wilt G-J, Thunnissen FBJM, et al. A prospective study of the timing and cost-effectiveness of bronchial washing during bronchoscopy for pulmonary malignant tumors. Chest 2005;128:394-400.

153 Bradley B, Branley HM, Egan JJ, et al. Interstitial lung disease guideline: the British Thoracic Society in collaboration with the Thoracic Society of Australia and New Zealand and the Irish Thoracic Society. Thorax 2008;63(Suppl 5):v1-58.

154 Reynolds HY. Present status of bronchoalveolar lavage in interstitial lung disease. Curr Opin Pulm Med 2009;15:479-85.

155 Leonard C, Tormey VJ, O'Keane C, et al. Bronchoscopic diagnosis of sarcoidosis. Eur Respir J 1997;10:2722-4.

156 Agusti C, Xaubet A, Arriols R, et al. Multinuclear giant cells in bronchoalveolar lavage in interstitial lung diseases. Respiration 1987;51:307-11.

157 Puar HS, Young RC Jr, Armstrong EM. Bronchial and transbronchial lung biopsy without fluoroscopy in sarcoidosis. Chest 1985;87:303-6.

158 Milman N, Graudal N, Jacobsen GK. Bronchoalveolar lavage in radiologically detected diffuse lung disease. Diagnostic value of total and differential cell count in a series of 130 patients. APMIS 1995;103:764-8

159 Mitchell DM, Emerson CJ, Collins JV, et al. Transbronchial lung biopsy with the fibreoptic bronchoscope: analysis of results in 433 patients. Br J Dis Chest 1981;75:258-62.

160 Shorr AF, Torrington KG, Hnatiuk OW. Endobronchial biopsy for sarcoidosis: a prospective study. Chest 2001:120:109-14 
161 Velardocchio JM, Boutin C, Irisson M. Broncho alveolar lavage in diffuse lung diseases, comparison with transbronchial and thoracoscopic lung biopsy. Eur $J$ Respir Dis 1983:62(Suppl 126):457-8.

162 Rohatgi PK, Kuzmowych TV, Delaney MD. Indications for transbronchial lung biopsy in the diagnosis of intrathoracic sarcoidosis. Respiration 1981:42:155-60.

163 Navani N, Booth HL, Kocjan G, et al. Combination of endobronchial ultrasound-guided transbronchial needle aspiration with standard bronchoscopic techniques for the diagnosis of stage I and stage II pulmonary sarcoidosis. Respirology 2011;16:467-72.

164 Descombes E, Gardiol D, Leuenberger P. Transbronchial lung biopsy: an analysis of 530 cases with reference to the number of samples. Monaldi Arch Chest Dis 1997:52:324-9.

165 Anders GT, Johnson JE, Bush BA, et al. Transbronchial biopsy without fluoroscopy. A seven-year perspective. Chest 1988;94:557-60.

166 Rhee CK, Kang HH, Kang JY, et al. Diagnostic yield of flexible bronchoscopy without fluoroscopic guidance in evaluating peripheral lung lesions. J Bronchol 2010;17:317-22

167 Chechani V. Bronchoscopic diagnosis of solitary pulmonary nodules and lung masses in the absence of endobronchial abnormality. Chest 1996;109:620-5.

168 de Fenoyl O, Capron F, Lebeau B, et al. Transbronchial biopsy without fluoroscopy: a five year experience in outpatients. Thorax 1989:44:956-9.

169 McLeod DT, Neill P, Gwanzura L, et al. Pneumocystis carinii pneumonia in patients with AIDS in Central Africa. Respir Med 1990;84:225-8.

170 Malin AS, Gwanzura LK, Klein S, et al. Pneumocystis carinii pneumonia in Zimbabwe. Lancet 1995:346:1258-61.

171 Abramson MJ, Stone CA, Holmes PW, et al. The role of bronchoalveolar lavage in the diagnosis of suspected opportunistic pneumonia. Aust N Z J Med 1987;17:407-12.

172 Rano A, Agusti C, Jimenez P, et al. Pulmonary infiltrates in non-HIV immunocompromised patients: a diagnostic approach using non-invasive and bronchoscopic procedures. Thorax 2001;56:379-87.

173 Jensen BN, Gerstoft J, Hojlyng N, et al. Pulmonary pathogens in HIV-infected patients. Scand J Infect Dis 1990:22:413-20.

174 Golden JA, Hollander H, Stulbarg MS, et al. Bronchoalveolar lavage as the exclusive diagnostic modality for Pneumocystis carinii pneumonia. A prospective study among patients with acquired immunodeficiency syndrome. Chest 1986;90:18-22

175 Heurlin N, Elvin K, Lidman C, et al. Fiberoptic bronchoscopy and sputum examination for diagnosis of pulmonary disease in AIDS patients in Stockholm. Scand J Infect Dis 1990;22:659-64.

176 Orenstein M, Webber CA, Cash M, et al. Value of bronchoalveolar lavage in the diagnosis of pulmonary infection in acquired immune deficiency syndrome. Thorax 1986;41:345-9.

177 Chuang MT, Rosen MJ, Teirstein AS, et al. Flexible bronchoscopy in the diagnosis of Pneumocystis carinii pneumonia in patients with acquired immune deficiency syndrome. South Med J 1986:79:1363-5.

178 Gracia JD, Miravitlles M, Mayordomo C, et al. Empiric treatments impair the diagnostic yield of BAL in HIV-positive patients. Chest 1997:111:1180-6.

179 Meduri GU, Stover DE, Greeno RA, et al. Bilateral bronchoalveolar lavage in the diagnosis of opportunistic pulmonary infections. Chest 1991;100:1272-6.

180 Baughman RP, Dohn MN, Shipley R, et al. Increased Pneumocystis carinii recovery from the upper lobes in Pneumocystis pneumonia. The effect of aerosol pentamidine prophylaxis. Chest 1993:103:426-32.

181 Read CA, Cerrone F, Busseniers AE, et al. Differential lobe lavage for diagnosis of acute Pneumocystis carinii pneumonia in patients receiving prophylactic aerosolized pentamidine therapy. Chest 1993;103:1520-3.

182 Salzman SH, Schindel ML, Aranda CP, et al. The role of bronchoscopy in the diagnosis of pulmonary tuberculosis in patients at risk for HIV infection. Chest 1992;102:143-6.

183 Kennedy DJ, Lewis WP, Barnes PF. Yield of bronchoscopy for the diagnosis of tuberculosis in patients with human immunodeficiency virus infection. Chest 1992:102:1040-4.

184 Miro AM, Gibilara E, Powell S, et al. The role of fiberoptic bronchoscopy for diagnosis of pulmonary tuberculosis in patients at risk for AIDS. Chest 1992; 101:1211-14

185 Kibiki GS, Mulder B, van der Ven AJ, et al. Laboratory diagnosis of pulmonary tuberculosis in TB and HIV endemic settings and the contribution of real time PCR for M. tuberculosis in bronchoalveolar lavage fluid. Trop Med Int Health 2007; 12:1210-17.

186 Baughman RP, Dohn MN, Loudon RG, et al. Bronchoscopy with bronchoalveolar lavage in tuberculosis and fungal infections. Chest 1991;99:92-7.

187 Levy H, Horak DA, Tegtmeier BR, et al. The value of bronchoalveolar lavage and bronchial washings in the diagnosis of invasive pulmonary aspergillosis. Respir Med 1992;86:243-8.

188 Kahn FW, Jones JM, England DM. The role of bronchoalveolar lavage in the diagnosis of invasive pulmonary aspergillosis. Am J Clin Pathol 1986;86:518-23.

189 Guo YL, Chen YQ, Wang K, et al. Accuracy of BAL galactomannan in diagnosing invasive aspergillosis: a bivariate metaanalysis and systematic review. Chest 2010;138:817-24
190 Meersseman W, Lagrou K, Maertens J, et al. Galactomannan in bronchoalveolar lavage fluid: a tool for diagnosing aspergillosis in intensive care unit patients. Am J Respir Crit Care Med 2008;177:27-34.

191 Mattei D, Rapezzi D, Mordini N, et al. False-positive Aspergillus galactomannan enzyme-linked immunosorbent assay results in vivo during amoxicillin-clavulanic acid treatment. J Clin Microbiol 2004;42:5362-3.

192 Adam O, Auperin A, Wilquin F, et al. Treatment with piperacillin-tazobactam and false-positive Aspergillus galactomannan antigen test results for patients with hematological malignancies. Clin Infect Dis 2004;38:917-20.

193 Viscoli C, Machetti M, Cappellano P, et al. False-positive galactomannan platelia Aspergillus test results for patients receiving piperacillin-tazobactam. Clin Infect Dis 2004:38:913-6.

194 Aubry A, Porcher R, Bottero J, et al. Occurrence and kinetics of false-positive Aspergillus galactomannan test results following treatment with beta-lactam antibiotics in patients with hematological disorders. J Clin Microbiol 2006;44:389-94.

195 Luong ML, Clancy CJ, Vadnerkar A, et al. Comparison of an Aspergillus real-time polymerase chain reaction assay with galactomannan testing of bronchoalvelolar lavage fluid for the diagnosis of invasive pulmonary aspergillosis in lung transplant recipients. Clin Infect Dis 52:1218-26.

196 Musher B, Fredricks D, Leisenring W, et al. Aspergillus galactomannan enzyme immunoassay and quantitative PCR for diagnosis of invasive aspergillosis with bronchoalveolar lavage fluid. J Clin Microbiol 2004:42:5517-22.

197 Lewin SR, Hoy J, Crowe SM, et al. The role of bronchoscopy in the diagnosis and treatment of pulmonary disease in HIV-infected patients. Aust N Z J Med 1995;25:133-9.

198 Malabonga VM, Basti J, Kamholz SL. Utility of bronchoscopic sampling techniques for cryptococcal disease in AIDS. Chest 1991;99:370-2.

199 Dugan JM, Avitabile AM, Rossman MD, et al. Diagnosis of Pneumocystis carinii pneumonia by cytologic evaluation of Papanicolaou-stained bronchial specimens. Diagn Cytopathol 1988;4:106-12.

200 Hartman B, Koss M, Hui A, et al. Pneumocystis carinii pneumonia in the acquired immunodeficiency syndrome (AIDS). Diagnosis with bronchial brushings, biopsy, and bronchoalveolar lavage. Chest 1985;87:603-7.

201 Albelda SM, Talbot GH, Gerson SL, et al. Role of fiberoptic bronchoscopy in the diagnosis of invasive pulmonary aspergillosis in patients with acute leukemia. Am J Med 1984;76:1027-34

202 Lim WS, Baudouin SV, George RC, et al. BTS guidelines for the management of community acquired pneumonia in adults: update 2009. Thorax 2009;64(Suppl 3): iii $1-55$.

203 Mandell LA, Wunderink RG, Anzueto A, et al. Infectious Diseases Society of America/American Thoracic Society consensus guidelines on the management of community-acquired pneumonia in adults. Clin Infect Dis 2007;44(Suppl 2):S27-72.

204 Gibson SP, Weir DC, Burge PS. A prospective audit of the value of fibre optic bronchoscopy in adults admitted with community acquired pneumonia. Respir Med 1993;87:105-9.

205 Dikensoy O, Usalan C, Filiz A. Foreign body aspiration: clinical utility of flexible bronchoscopy. Postgrad Med J 2002;78:399-403.

206 Dasgupta KS, Mundada PS, Soni N. Diagnostic role of fibreoptic bronchoscopy in pulmonary tuberculosis. Indian J Otolaryngol Head Neck Surg 2000;52:347-9.

207 Jaiswal AK, Kulpati DD, Jain NK, et al. Role of bronchoscopy in the early diagnosis of suspected smear negative cases of pulmonary tuberculosis. Ind J Tub 1989;36:233.

208 de Gracia J, Curull V, Vidal R, et al. Diagnostic value of bronchoalveolar lavage in suspected pulmonary tuberculosis. Chest 1988;93:329-32.

209 Altaf Bachh A, Gupta R, Haq I, et al. Diagnosing sputum/smear-negative pulmonary tuberculosis: does fibre-optic bronchoscopy play a significant role? Lung India 2010;27:58-62.

210 Bartlett JG, Alexander J, Mayhew J, et al. Should fiberoptic bronchoscopy aspirates be cultured? Am Rev Respir Dis 1976;114:73-8.

211 Wimberley N, Faling LJ, Bartlett JG. A fiberoptic bronchoscopy technique to obtain uncontaminated lower airway secretions for bacterial culture. Am Rev Respir Dis 1979;119:337-43.

212 Manali E, Papadopoulos A, Tsiodras S, et al. The impact on community acquired pneumonia empirical therapy of diagnostic bronchoscopic techniques. Scand J Infect Dis 2008;40:286-92.

213 Jimenez $\mathrm{P}$, Saldias F, Meneses $\mathrm{M}$, et al. Diagnostic fiberoptic bronchoscopy in patients with community-acquired pneumonia. Comparison between bronchoalveolar lavage and telescoping plugged catheter cultures. Chest 1993:103:1023-7.

214 Ortqvist A, Kalin M, Lejdeborn L, et al. Diagnostic fiberoptic bronchoscopy and protected brush culture in patients with community-acquired pneumonia. Chest 1990;97:576-82.

215 Jimenez $\mathrm{P}$, Meneses M, Saldias F, et al. Pneumococcal antigen detection in bronchoalveolar lavage fluid from patients with pneumonia. Thorax 1994;49:872-4.

216 Jacobs JA, Stobberingh EE, Cornelissen El, et al. Detection of Streptococcus pneumoniae antigen in bronchoalveolar lavage fluid samples by a rapid immunochromatographic membrane assay. J Clin Microbiol 2005;43:4037-40. 
217 Jaulhac B, Nowicki M, Bornstein N, et al. Detection of Legionella spp. in bronchoalveolar lavage fluids by DNA amplification. J Clin Microbio/ 1992;30:920-4.

218 Reischl U, Linde HJ, Lehn N, et al. Direct detection and differentiation of Legionella spp. and Legionella pneumophila in clinical specimens by dual-color real-time PCR and melting curve analysis. J Clin Microbiol 2002;40:3814-17.

219 Charoenratanakul S, Dejsomritrutai W, Chaiprasert A. Diagnostic role of fiberoptic bronchoscopy in suspected smear negative pulmonary tuberculosis. Respir Med 1995;89:621-3

220 Liam CK, Chen YC, Yap SF, et al. Detection of Mycobacterium tuberculosis in bronchoalveolar lavage from patients with sputum smear-negative pulmonary tuberculosis using a polymerase chain reaction assay. Respirology 1998;3:125-9.

221 Tueller C, Chhajed PN, Buitrago-Tellez C, et al. Value of smear and PCR in bronchoalveolar lavage fluid in culture positive pulmonary tuberculosis. Eur Respir $\mathrm{J}$ 2005:26:767-72.

222 Jafari C, Thijsen S, Sotgiu G, et al. Bronchoalveolar lavage enzyme-linked immunospot for a rapid diagnosis of tuberculosis: a Tuberculosis Network European Trials group study. Am J Respir Crit Care Med 2009;180:666-73.

223 Jafari C, Ernst M, Kalsdorf B, et al. Rapid diagnosis of smear-negative tuberculosis by bronchoalveolar lavage enzyme-linked immunospot. Am J Respir Crit Care Med 2006;174:1048-54.

224 Fujii $\mathrm{H}$, Ishihara J, Fukaura A, et al. Early diagnosis of tuberculosis by fibreoptic bronchoscopy. Tuber Lung Dis 1992;73:167-9.

225 Brimacombe J, Dunbar-Reid K. The effect of introducing fibreoptic bronchoscopes on gas flow in laryngeal masks and tracheal tubes. Anaesthesia 1996;51:923-8.

226 Haenel JB, Moore FA, Moore EE, et al. Efficacy of selective intrabronchial air insufflation in acute lobar collapse. Am J Surg 1992;164:501-5.

227 Harada K, Mutsuda T, Saoyama N, et al. Re-expansion of refractory atelectasis using a bronchofiberscope with a balloon cuff. Chest 1983;84:725-8.

228 Lee TS, Wright BD. Selective insufflation of collapsed lung with fiberoptic bronchoscope and Swan-Ganz catheter. Intensive Care Med 1981;7:241-3.

229 Tsao TC, Tsai YH, Lan RS, et al. Treatment for collapsed lung in critically ill patients. Selective intrabronchial air insufflation using the fiberoptic bronchoscope. Chest 1990;97:435-8.

230 Tabboush ZS, Ayash RH, Badran HM. When fiberoptic bronchoscopy is indicated in the management of postoperative atelectasis. Acta Anaesthesiol Scand 1998:42:384

231 Jaworski A, Goldberg SK, Walkenstein MD, et al. Utility of immediate postlobectomy fiberoptic bronchoscopy in preventing atelectasis. Chest 1988;94:38-43.

232 Dupree HJ, Lewejohann JC, Gleiss J, et al. Fiberoptic bronchoscopy of intubated patients with life-threatening hemoptysis. World J Surg 2001;25:104-7.

233 Khalil A, Soussan M, Mangiapan G, et al. Utility of high-resolution chest CT scan in the emergency management of haemoptysis in the intensive care unit: severity, localization and aetiology. Br J Radiol 2007;80:21-5.

234 Baigelman W, Bellin S, Cupples LA, et al. Bacteriologic assessment of the lower respiratory tract in intubated patients. Crit Care Med 1986;14:864-8.

235 Barreiro B, Dorca J, Manresa F, et al. Protected bronchoalveolar lavage in the diagnosis of ventilator-associated pneumonia. Eur Respir J 1996;9:1500-7.

236 Bello S, Tajada A, Chacon E, et al. 'Blind' protected specimen brushing versus bronchoscopic techniques in the aetiolological diagnosis of ventilator-associated pneumonia. Eur Respir J 1996;9:1494-9.

237 Casetta M, Blot F, Antoun S, et al. Diagnosis of nosocomial pneumonia in cancer patients undergoing mechanical ventilation: a prospective comparison of the plugged telescoping catheter with the protected specimen brush. Chest 1999;115:1641-5.

238 Humphreys H, Winter R, Baker M, et al. Comparison of bronchoalveolar lavage and catheter lavage to confirm ventilator-associated lower respiratory tract infection. J Med Microbiol 1996;45:226-31.

239 Jorda R, Parras F, Ibanez J, et al. Diagnosis of nosocomial pneumonia in mechanically ventilated patients by the blind protected telescoping catheter. Intensive Care Med 1993;19:377-82.

240 Jourdain B, Novara A, Joly-Guillou ML, et al. Role of quantitative cultures of endotracheal aspirates in the diagnosis of nosocomial pneumonia. Am J Respir Crit Care Med 1995;152:241-6.

241 Kirtland SH, Corley DE, Winterbauer RH, et al. The diagnosis of ventilator-associated pneumonia: a comparison of histologic, microbiologic, and clinical criteria. Chest 1997;112:445-57.

242 Lambert RS, Vereen LE, George RB. Comparison of tracheal aspirates and protected brush catheter specimens for identifying pathogenic bacteria in mechanically ventilated patients. Am J Med Sci 1989;297:377-82.

243 Leal-Noval SR, Alfaro-Rodriguez E, Murillo-Cabeza F, et al. Diagnostic value of the blind brush in mechanically ventilated patients with nosocomial pneumonia. Intensive Care Med 1992;18:410-14.

244 Marquette $\mathrm{CH}$, Herengt $\mathrm{F}$, Mathieu $\mathrm{D}$, et al. Diagnosis of pneumonia in mechanically ventilated patients. Repeatability of the protected specimen brush. Am Rev Respir Dis 1993;147:211-14.

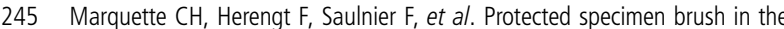
assessment of ventilator-associated pneumonia. Selection of a certain lung segment for bronchoscopic sampling is unnecessary. Chest 1993;103:243-7.
246 Pham LH, Brun-Buisson C, Legrand P, et al. Diagnosis of nosocomial pneumonia in mechanically ventilated patients. Comparison of a plugged telescoping catheter with the protected specimen brush. Am Rev Respir Dis 1991;143(5 Pt 1):1055-61.

247 Potgieter PD, Hammond JM. Etiology and diagnosis of pneumonia requiring ICU admission. Chest 1992;101:199-203.

248 Pugin J, Auckenthaler R, Mili N, et al. Diagnosis of ventilator-associated pneumonia by bacteriologic analysis of bronchoscopic and nonbronchoscopic 'blind' bronchoalveolar lavage fluid. Am Rev Respir Dis 1991;143(5 Pt 1):1121-9.

249 Rodriguez de Castro F, Sole-Violan J, Aranda Leon A, et al. Do quantitative cultures of protected brush specimens modify the initial empirical therapy in ventilated patients with suspected pneumonia? Eur Respir J 1996;9:37-41.

250 Rouby JJ, Rossignon MD, Nicolas MH, et al. A prospective study of protected bronchoalveolar lavage in the diagnosis of nosocomial pneumonia. Anesthesiology 1989:71:679-85.

251 Sanchez-Nieto JM, Torres A, Garcia-Cordoba F, et al. Impact of invasive and noninvasive quantitative culture sampling on outcome of ventilator-associated pneumonia: a pilot study. Am J Respir Crit Care Med 1998;157:371-6.

252 Souweine B, Veber B, Bedos JP, et al. Diagnostic accuracy of protected specimen brush and bronchoalveolar lavage in nosocomial pneumonia: impact of previous antimicrobial treatments. Crit Care Med 1998;26:236-44

253 Swanson JM, Wood GC, Croce MA, et al. Utility of preliminary bronchoalveolar lavage results in suspected ventilator-associated pneumonia. J Trauma 2008;65:1271-7.

254 Timsit JF, Misset B, Francoual S, et al. Is protected specimen brush a reproducible method to diagnose ICU-acquired pneumonia? Chest 1993;104:104-8.

255 Timsit JF, Misset B, Renaud B, et al. Effect of previous antimicrobial therapy on the accuracy of the main procedures used to diagnose nosocomial pneumonia in patients who are using ventilation. Chest 1995;108:1036-40.

256 Torres A, el-Ebiary M, Padro L, et al. Validation of different techniques for the diagnosis of ventilator-associated pneumonia. Comparison with immediate postmortem pulmonary biopsy. Am J Respir Crit Care Med 1994;149(2 Pt 1):324-31.

257 Torres A, Puig de la Bellacasa J, Rodriguez-Roisin R, et al. Diagnostic value of telescoping plugged catheters in mechanically ventilated patients with bacterial pneumonia using the Metras catheter. Am Rev Respir Dis 1988;138:117-20.

258 Berton DC, Kalil AC, Cavalcanti M, et al. Quantitative versus qualitative cultures of respiratory secretions for clinical outcomes in patients with ventilator-associated pneumonia. Cochrane Database Syst Rev 2008;(4):CD006482.

259 Berton DC, Kalil AC, Teixeira PJ. Quantitative versus qualitative cultures of respiratory secretions for clinical outcomes in patients with ventilator-associated pneumonia. Cochrane Database Syst Rev 2012;(1):CD006482.

260 Pincus PS, Kallenbach JM, Hurwitz MD, et al. Transbronchial biopsy during mechanical ventilation. Crit Care Med 1987;15:1136-9.

261 Ghamande S, Rafanan A, Dweik R, et al. Role of transbronchial needle aspiration in patients receiving mechanical ventilation. Chest 2002;122:985-9.

262 Perkins GD, Chatterjie S, McAuley DF, et al. Role of nonbronchoscopic lavage for investigating alveolar inflammation and permeability in acute respiratory distress syndrome. Crit Care Med 2006;34:57-64.

$263 \mathrm{Kim}$ YH, Suh GY, Kim MH, et al. Safety and usefulness of bronchoscopy in ventilator-dependent patients with severe thrombocytopenia. Anaesth Intensive Care 2008:36:411-17.

264 Steinberg KP, Mitchell DR, Maunder RJ, et al. Safety of bronchoalveolar lavage in patients with adult respiratory distress syndrome. Am Rev Respir Dis 1993:148:556-61.

265 Bauer TT, Torres A, Ewig S, et al. Effects of bronchoalveolar lavage volume on arterial oxygenation in mechanically ventilated patients with pneumonia. Intensive Care Med 2001;27:384-93.

266 Hilbert G, Gruson D, Vargas F, et al. Bronchoscopy with bronchoalveolar lavage via the laryngeal mask airway in high-risk hypoxemic immunosuppressed patients. Crit Care Med 2001;29:249-55

267 Klein U, Karzai W, Zimmermann P, et al. Changes in pulmonary mechanics after fiberoptic bronchoalveolar lavage in mechanically ventilated patients. Intensive Care Med 1998;24:1289-93.

268 Maitre B, Jaber S, Maggiore SM, et al. Continuous positive airway pressure during fiberoptic bronchoscopy in hypoxemic patients. A randomized double-blind study using a new device. Am J Respir Crit Care Med 2000;162(3 Pt 1):1063-7.

269 Papazian L, Colt HG, Scemama F, et al. Effects of consecutive protected specimen brushing and bronchoalveolar lavage on gas exchange and hemodynamics in ventilated patients. Chest 1993;104:1548-52.

270 Previgliano IJ, Ripoll PI, Chiappero G, et al. Optimizing cerebral perfusion pressure during fiberoptic bronchoscopy in severe head injury: effect of hyperventilation. Acta Neurochir Supp/ 2002;81:103-5.

271 Trouillet JL, Guiguet M, Gibert C, et al. Fiberoptic bronchoscopy in ventilated patients. Evaluation of cardiopulmonary risk under midazolam sedation. Chest 1990;97:927-33.

272 Maclntyre NR, Ramage JE, Follett JV. Jet ventilation in support of fiberoptic bronchoscopy. Crit Care Med 1987;15:303-7.

273 Ramanathan S, Sinha K, Arismendy J, et al. Humidification and airway pressures during high-frequency jet ventilation delivered through the suction-biopsy channel of a flexible bronchofiberscope. Crit Care Med 1984;12:820-3. 
274 Flatau E, Lewinsohn G, Konichezky S, et al. Mechanical ventilation in fiberoptic-bronchoscopy: comparison between high frequency positive pressure ventilation and normal frequency positive pressure ventilation. Crit Care Med 1982;10:733-5.

275 Antonelli M, Conti G, Riccioni L, et al. Noninvasive positive-pressure ventilation via face mask during bronchoscopy with BAL in high-risk hypoxemic patients. Chest 1996;110:724-8.

276 Chiner E, Sancho-Chust JN, Llombart M, et al. Fiberoptic bronchoscopy during nasal non-invasive ventilation in acute respiratory failure. Respiration 2010;80:321-6

277 Baumann HJ, Klose $\mathrm{H}$, Simon M, et al. Fiber optic bronchoscopy in patients with acute hypoxemic respiratory failure requiring noninvasive ventilation - a feasibility study. Crit Care 2011;15:R179.

278 Bajwa MK, Henein S, Kamholz SL. Fiberoptic bronchoscopy in the presence of space-occupying intracranial lesions. Chest 1993;104:101-3.

279 Kerwin AJ, Croce MA, Timmons SD, et al. Effects of fiberoptic bronchoscopy on intracranial pressure in patients with brain injury: a prospective clinical study. $J$ Trauma 2000:48:878-82; discussion 882-3.

280 Peerless JR, Snow N, Likavec MJ, et al. The effect of fiberoptic bronchoscopy on cerebral hemodynamics in patients with severe head injury. Chest 1995;108:962-5.

281 Cleaning and disinfection of equipment for gastrointestinal endoscopy. Report of a Working Party of the British Society of Gastroenterology Endoscopy Committee. Gut 1998:42:585-93.

282 Beilenhoff U, Neumann CS, Rey JF, et al. The ESGE Guidelines Committee. ESGEESGENA guideline: cleaning and disinfection in gastrointestinal endoscopy. Update 2008. Endoscopy 2008;40:939-57.

283 National Endoscopy Programme. Decontamination standards for flexible endoscopes 2008. 2009. http://www.thejag.org.uk/downloads\%5CUnit\% 20Resources $\% 5$ CDecontamination $\% 20$ Standards $\% 20$ for $\% 20$ Flexible $\%$ 20Endoscopy.pdf (accessed Jun 2013).

284 MHRA. Medical Devices Agency (MDA) Device Bulletin DB2002 (05) on decontamination of endoscopes. 2005. http://www.mhra.gov.uk/Publications/ Safetyguidance/DeviceBulletins/CON007329

285 MHRA. MDA/2004/028 - Flexible and rigid endoscopes. June 2004. http://www. mhra.gov.uk/Publications/Safetywarnings/MedicalDeviceAlerts/CON008543 (accessed Jun 2013).

286 The Health Act 2006: Code of practice for the prevention and control of healthcare associated infections. 2006. http://www.hpa.org.uk/Topics/InfectiousDiseases/ InfectionsAZ/HCAI/GuidelinesForProfessionalsHCAl/ (accessed Jun 2013).

287 Niven K. An evaluation of chemical disinfecting agents used in endoscopy suites in the NHS. 2007. http://www.hse.gov.uk/research/rrpdf/rr445.pdf (accessed Jun 2013).
288 Health and Safety Executive (HSE) UK. An evaluation of disinfecting agents used in endoscopy suites. 2007. http://www.hse.gov.uk/research/rrhtm/rr445.htm (accessed Jun 2013).

289 British Society of Gastroenterology Provision of Endoscopy Related Services in District General Hospitals. 2001. http://www.bsg.org.uk/images/stories/docs/ clinical/guidelines/endoscopy/endo_related_services.pdf (accessed Jun 2013).

290 Poi PJ, Chuah SY, Srinivas P, et al. Common fears of patients undergoing bronchoscopy. Eur Respir J 1998;11:1147-9.

291 Diette GB, White P Jr, Terry P, et al. Quality assessment through patient self-report of symptoms prefiberoptic and postfiberoptic bronchoscopy. Chest 1998; 114:1446-53.

292 Lechtzin N, Rubin HR, White P Jr, et al. Patient satisfaction with bronchoscopy. Am J Respir Crit Care Med 2002;166:1326-31.

293 Hirose $\mathrm{T}$, Okuda $\mathrm{K}$, Ishida $\mathrm{H}$, et al. Patient satisfaction with sedation for flexible bronchoscopy. Respirology 2008;13:722-7.

294 Hadzri H, Azarisman S, Fauzi A, et al. Can a bronchoscopist reliably assess a patient's experience of bronchoscopy? JRSM Short Rep 2010;1:35.

295 Bernasconi M, Chhajed PN, Muller P, et al. Patients' satisfaction with flexible bronchoscopy in a hospital-based community practice. Respiration 2009;78:440-5.

296 Mitsumune T, Senoh E, Adachi M. Prediction of patient discomfort during fibreoptic bronchoscopy. Respirology 2005;10:92-6.

297 Diette GB, Lechtzin N, Haponik E, et al. Distraction therapy with nature sights and sounds reduces pain during flexible bronchoscopy: a complementary approach to routine analgesia. Chest 2003;123:941-8.

298 Dubois JM, Bartter T, Pratter MR. Music improves patient comfort level during outpatient bronchoscopy. Chest 1995;108:129-30.

299 Colt HG, Powers A, Shanks TG. Effect of music on state anxiety scores in patients undergoing fiberoptic bronchoscopy. Chest 1999;116:819-24.

300 Brady M, Kinn S, Stuart P. Preoperative fasting for adults to prevent perioperative complications. Cochrane Database Syst Rev 2003;(4):CD004423.

301 Molina JA, Lobo CA, Goh HK, et al. Review of studies and guidelines on fasting and procedural sedation at the emergency department. Int J Evid Based Healthc 2010;8:75-8.

302 Department of Health UK. Understanding what matters: a guide to using patient feedback to transform care. May 2009. http://webarchive.nationalarchives.gov.uk +/www.dh.gov.uk/en/Publicationsandstatistics/Publications/ PublicationsPolicyAndGuidance/DH_099780

303 General Medical Council UK. Consent guidance: patients and doctors making decisions together. 2008. http://www.gmc-uk.org/guidance/ethical_guidance/ consent_guidance_index.asp 


\section{APPENDIX 1 CONTRIBUTIONS AND DECLARATIONS OF INTEREST}

\begin{tabular}{|c|c|c|}
\hline Name & Affiliation & Declaration of interest \\
\hline $\begin{array}{l}\text { Dr IA Du Rand } \\
\text { Consultant Physician in Respiratory Medicine with a special } \\
\text { interest in lung cancer and evidence based medicine } \\
\text { Worcestershire Acute Hospitals NHS Trust } \\
\text { MSc University of Oxford, Evidence Based Health Care }\end{array}$ & $\begin{array}{l}\text { Worcestershire Royal Hospital, Worcestershire } \\
\text { Acute Hospitals NHS Trust, Worcester, UK }\end{array}$ & None \\
\hline $\begin{array}{l}\text { Dr J Blaikley } \\
\text { Academic Clinical Lecturer and specialist registrar in } \\
\text { Respiratory Medicine } \\
\text { The University of Manchester }\end{array}$ & University of Manchester, Manchester, UK & None \\
\hline $\begin{array}{l}\text { R Booton } \\
\text { Consultant Physician in Respiratory Medicine and Clinical } \\
\text { Senior Lecturer in Respiratory Medicine Respiratory Research } \\
\text { Group, School of Translational Medicine, The University of } \\
\text { Manchester }\end{array}$ & $\begin{array}{l}\text { The University of Manchester, Manchester } \\
\text { Academic Health Science Centre, University } \\
\text { Hospital South Manchester NHS Foundation Trust, } \\
\text { Manchester, UK }\end{array}$ & None \\
\hline $\begin{array}{l}\text { N Chaudhuri } \\
\text { Consultant Physician in Respiratory Medicine } \\
\text { United Hospital of South manchester, Wythenshawe } \\
\text { hospital, Manchester. }\end{array}$ & $\begin{array}{l}\text { University Hospital of South Manchester, } \\
\text { Manchester, UK }\end{array}$ & None \\
\hline $\begin{array}{l}\text { V Gupta } \\
\text { Academic Clinical Lecturer and specialist registrar in } \\
\text { Respiratory Medicine } \\
\text { The University of Manchester }\end{array}$ & University of Manchester, Manchester, UK & None \\
\hline $\begin{array}{l}\text { S Khalid } \\
\text { Consultant Physician in Respiratory Medicine } \\
\text { Royal Blackburn Hospital, Lancashire, UK }\end{array}$ & Royal Blackburn Hospital, Lancashire, UK & None \\
\hline $\begin{array}{l}\text { S Mandal } \\
\text { Specialist Registrar in Respiratory Medicine } \\
\text { Lane Fox Unit, St Thomas' Hospital, London, UK }\end{array}$ & Lane Fox Unit, St Thomas' Hospital, London, UK & None \\
\hline $\begin{array}{l}\text { J Martin } \\
\text { Consultant nurse bronchoscopist. } \\
\text { South Manchester University Hospital NHS Trust }\end{array}$ & $\begin{array}{l}\text { University Hospital of South Manchester, } \\
\text { Manchester, UK }\end{array}$ & None \\
\hline $\begin{array}{l}\text { J Mills } \\
\text { Endoscopy Nurse \& Respiratory Research Nurse } \\
\text { Lancashire Teaching Hospitals } \\
\text { Preston }\end{array}$ & $\begin{array}{l}\text { Lancashire Teaching Hospitals NHS Trust, Preston, } \\
\text { UK }\end{array}$ & None \\
\hline $\begin{array}{l}\text { N Navani } \\
\text { Consultant Physician in Respiratory Medicine } \\
\text { University College London Hospital \& MRC Clinical Trials } \\
\text { Unit, London }\end{array}$ & $\begin{array}{l}\text { University College London Hospital, National } \\
\text { Institute for Health Research University College } \\
\text { London Hospitals Biomedical Research Centre, } \\
\text { London, UK }\end{array}$ & $\begin{array}{l}\text { Dr Navani is chair of the EBUS Live courses, sponsored } \\
\text { by Olympus. He has no financial interest in Olympus and } \\
\text { there is no conflict of interest in relation to these } \\
\text { guidelines. }\end{array}$ \\
\hline $\begin{array}{l}\text { NM Rahman } \\
\text { Consultant Physician in Respiratory Medicine and Senior } \\
\text { Lecturer } \\
\text { NIHR Oxford Biomedical Research Centre, University of } \\
\text { Oxford and Oxford Centre for Respiratory Medicine, Churchill } \\
\text { Hospital, Oxford }\end{array}$ & $\begin{array}{l}\text { Oxford Centre for Respiratory Medicine, NIHR } \\
\text { Oxford Biomedical Research Centre, Oxford } \\
\text { Respiratory Trials Unit, University of Oxford, } \\
\text { Oxford, UK }\end{array}$ & $\begin{array}{l}\text { Involved in evaluation of interventional bronchoscopy/ } \\
\text { thoracoscopy related equipment for Olympus and } \\
\text { Spiration. Involved in Interventional Bronchoscopy/ } \\
\text { Thoracoscopy and Basic Bronchoscopy courses, which are } \\
\text { sponsored by numerous companies including Olympus, } \\
\text { ERBE, Wolf, Sonosite, Rocket, UK Medical, Nucleotron, } \\
\text { Pentax, Fujinon, Conmed, Cook, Spiration and others. No } \\
\text { financial interest in any of these companies and there is } \\
\text { no conflict of interest in relation to the guidelines. }\end{array}$ \\
\hline $\begin{array}{l}\text { JM Wrightson } \\
\text { Specialist Registrar in Respiratory Medicine and Clinical } \\
\text { Research Fellow } \\
\text { NIHR Oxford Biomedical Research Centre, University of } \\
\text { Oxford and Oxford Centre for Respiratory Medicine, Churchill } \\
\text { Hospital, Oxford }\end{array}$ & $\begin{array}{l}\text { Oxford Centre for Respiratory Medicine, NIHR } \\
\text { Oxford Biomedical Research Centre, Oxford } \\
\text { Respiratory Trials Unit, University of Oxford, } \\
\text { Oxford, UK }\end{array}$ & None \\
\hline $\begin{array}{l}\text { M Munavvar } \\
\text { Consultant Respiratory Physician with a special interest in } \\
\text { interventional bronchoscopy and lung cancer } \\
\text { Lancashire Teaching Hospitals NHS Trust Preston }\end{array}$ & $\begin{array}{l}\text { Lancashire Teaching Hospitals NHS Trust, Preston, } \\
\text { UK }\end{array}$ & $\begin{array}{l}\text { Dr M Munavvar has been involved in prototype } \\
\text { evaluation of interventional bronchoscopy/ thoracoscopy } \\
\text { related equipment for Olympus, Boston Scientific, Cook } \\
\text { and Spiration. He organises Interventional Bronchoscopy/ } \\
\text { Thoracoscopy and Basic Bronchoscopy courses, which are } \\
\text { sponsored by numerous companies including Olympus, } \\
\text { ERBE, Wolf, Sonosite, Rocket, UK Medical, Nucleotron, } \\
\text { Pentax, Fujinon, Conmed, Cook, Spiration and others. Dr } \\
\text { Munavvar has no financial interest in any of these } \\
\text { companies and there is no conflict of interest in relation } \\
\text { to the guidelines. }\end{array}$ \\
\hline Mrs A McCloy & Patient representative for this guideline & None \\
\hline
\end{tabular}


The assistance of the following colleagues is gratefully acknowledged:

- Nicholas Smith BA(Hons) MsC, Information Support Librarian.

Rowlands Library, Worcestershire Royal Hospital

- Emma Gibbs, Senior Library Assistant.

Rowlands Library, Worcestershire Royal Hospital.

- Philip Adams, Illustrator and artist

Master of Arts (Art Education)

- Prof Richard Lewis (Chairman) and the Guideline Committee of the BTS guideline for advanced diagnostic and therapeutic flexible bronchoscopy in adults.

- Sally Welham, Deputy Chief Executive of the BTS

\section{APPENDIX 2 - SEARCH STRATEGY: BRITISH THORACIC SOCIETY GUIDELINE FOR DIAGNOSTIC FLEXIBLE BRONCHOSCOPY IN ADULTS}

The search strategy is available on-line on the BTS website. Please also see Data supplement 1 (http://dx.doi.org/10.1136/ thoraxjnl-2013-203618)

\section{APPENDIX 3 THE EVIDENCE TABLES}

The evidence tables are available on-line on the BTS website for review. Please also see Data supplement 2 (http://dx.doi.org/ 10.1136/thoraxjnl-2013-203618)

\section{APPENDIX 4 LIST OF STAKEHOLDERS}

\section{List of Stakeholders}

Association of Respiratory Nurse Specialists

Association of Chartered Physiotherapists in Respiratory Care

Royal College of Physicians

Royal College of Physicians, Edinburgh

Royal College of Surgeons of England

Royal College of Surgeons of Edinburgh

Royal College of Physicians and Surgeons of Glasgow

Royal College of Anaesthetists

The Intensive Care Society

British Geriatrics Society

British Cardiovascular Society

Society for Acute Medicine

TSANZ

Endoscopy UK

Royal College of Nursing

Joint Royal Colleges Ambulance Liaison Committee

Royal College of General Practitioners

British Pharmacological Society

British Society for Rheumatology

British Infection Society

Association for Palliative Medicine of Great Britain and Ireland

The British Heart \& Lung Transplant Association

Royal College of Pathologists

Society for Cardiothoracic Surgery in GB and Ireland

College of Emergency Medicine

British Society for Immunology

The Association for Clinical Biochemistry

British Society of Clinical Cytology

Royal College of Radiologists

KeyMed Olympus, Pentax 


\section{APPENDIX 5: FLEXIBLE BRONCHOSCOPY SAFETY CHECKLIST}

Also available electronically on the BTS website

\section{$T$}

\section{BTS Flexible Bronchoscopy Safety Checklist (adapted from the WHO Surgical Safety Checklist)}
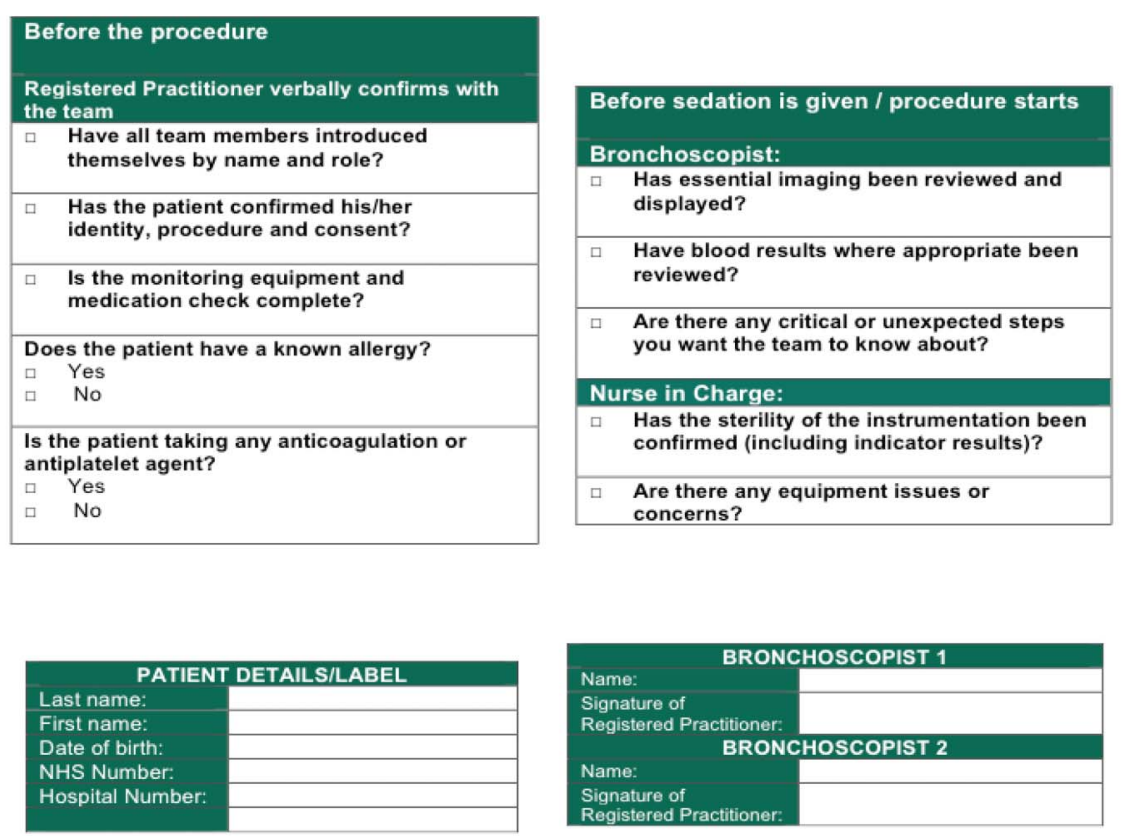
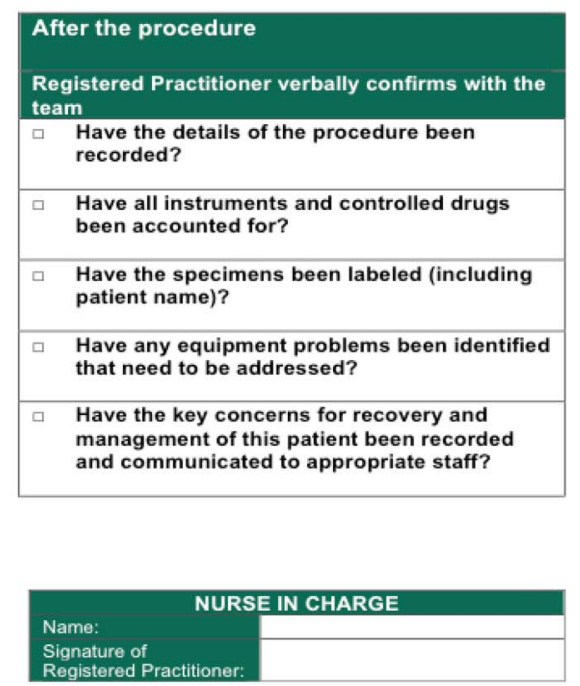

THIS CHECKLIST IS NOT INTENDED TO BE COMPREHENSIVE. ADDITIONS AND MODIFICATIONS TO FIT LOCAL PRACTICE ARE ENCOURAGED.

\section{APPENDIX 6: MANAGEMENT APPROACH TO BLEEDING AT BRONCHOSCOPY}

Major bleeding is uncommon following bronchoscopic biopsies but can complicate transbronchial lung biopsy or biopsy of some endobronchial tumours.

General Approach:

1. Consider whether the biopsy is necessary.

Only a few diagnoses can be reliably made with TBLB, and sampling of nodal or extra thoracic disease in malignancy may be more appropriate than endobronchial disease where staging information is required.

2. Does the patient have appropriate physiological reserve to withstand haemorrhage, and a secure patent airway

3. If bleeding is considered highly likely and biopsy urgent/ mandatory, ensure good IV access, commence supplemental oxygen, apply prophylactic local vasoconstrictor therapy and achieve the best first biopsy possible. Where available, the use of argon plasma coagulation or other similar haemostatic biopsy technique could be considered. Ensure senior advice/ assistance is available. As an alternative, consider a rigid bronchoscopic biopsy.

In the event of significant unexpected bleeding:

1. Ensure adequate oxygenation and IV access for fluid resuscitation. Vital signs should be monitored regularly.

2. Retract the bronchoscope proximally to maintain vision, and apply suction to remove free blood to preserve airway patency. Consider lying patient onto the side of the bleeding. Do not suction to remove clot.

3. Consider the application of local vasoconstrictor therapy. Agents include $5-10 \mathrm{ml} 1: 10000$ epinephrine or $5-10 \mathrm{ml} 4{ }^{\circ} \mathrm{C}$ saline. Saline has the advantage that it may be administered repeatedly.

4. If bleeding continues, the bronchoscope should be wedged into the bleeding segmental bronchus, if possible, and held in place for 10-15 minutes.

5. If this does not control the bleeding, a balloon catheter can be used to apply pressure and isolate the segment.

6. Check platelet count, PT and PTT, and recheck drug history.

7. Seek senior/expert assistance, and consider referral to critical care.

(TBLB : Transbronchial lung biopsy, PT: Prothrombin time, PTT: partial thromboplastin time) 


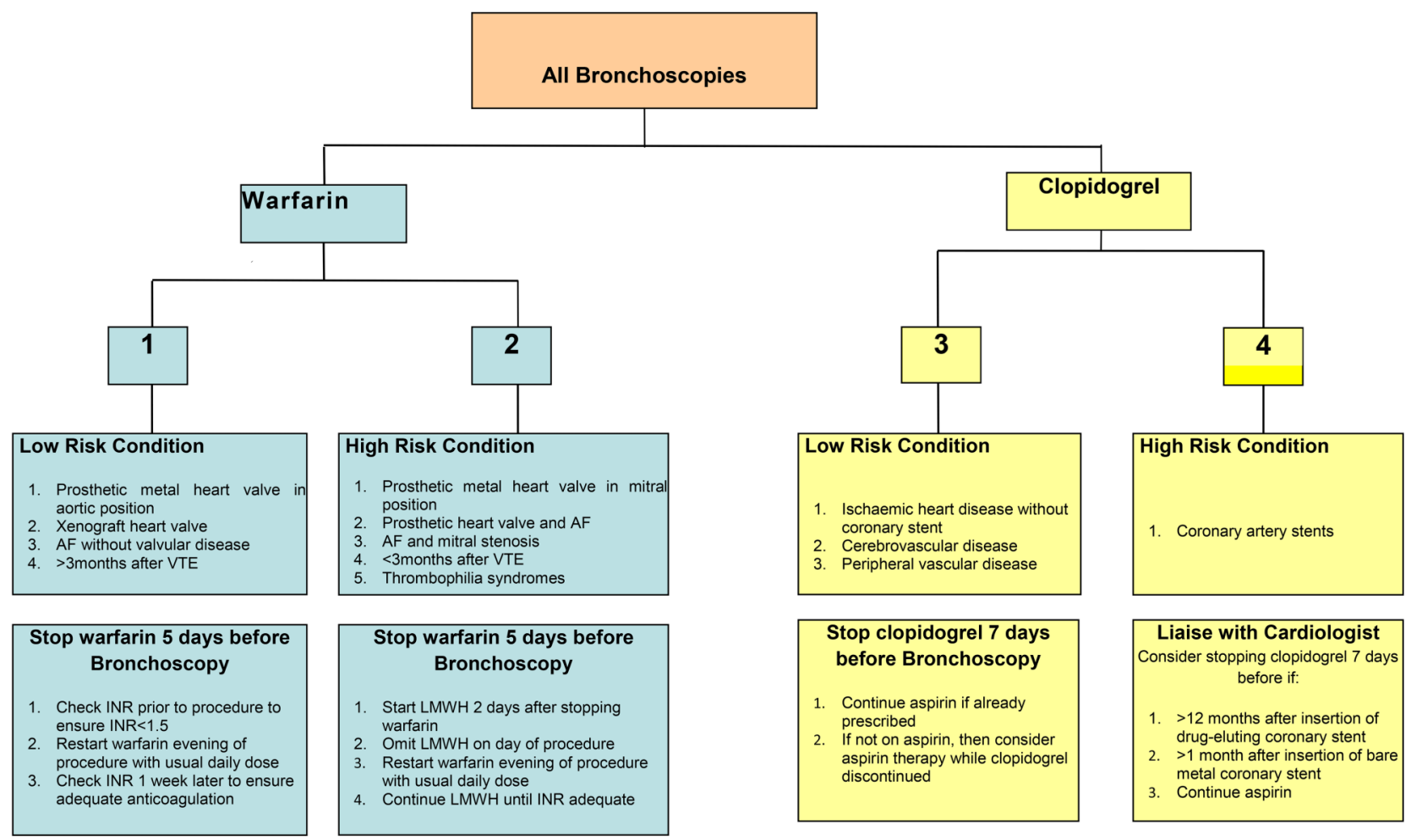

(INR: international normalised ratio, AF: atrial fibrillation, VTE: venous thromboembolism, LMWH: low molecular weight heparin)

Adapted from the Guidelines for the management of anticoagulant and antiplatelet therapy in patients undergoing endoscopic procedures. A M Veitch, T P Baglin, A H Gershlick, S M Harnden, R Tighe, S Cairns Gut 2008;57:9 1322-1329 Published Online First: 9 May 2008 doi:10.1136/gut.2007.142497 
APPENDIX 8: DRUGS USED IN BRONCHOSCOPY.

All tables are for guidance only; for up to date information on doses, side effects and interactions, refer to the British National Formulary.

Table 1 Commonly used drugs in bronchoscopy

\begin{tabular}{|c|c|c|c|c|}
\hline Drug & Dose & $\begin{array}{c}\text { Speed of Onset / Duration of } \\
\text { Action / Half-life }\end{array}$ & Common / Serious Side Effects & Comments \\
\hline Midazolam & $\begin{array}{l}\text { Slow IV injection - maximum rate } 2 \mathrm{mg} / \mathrm{min} \\
\text { Initial dose: } 2 \mathrm{mg}-2.5 \mathrm{mg}(0.5-1 \mathrm{mg} \text { in the frail or } \\
\text { elderly) given } 5-10 \text { mins before procedure } \\
\text { Supplemental doses, if required: } 1 \mathrm{mg} \text { (0.5-1 mg in } \\
\text { frail or elderly), at } 2-10 \text { mins intervals } \\
\text { Usual maximum total dose: } 3.5-7 \mathrm{mg} \text { ( } 3.5 \mathrm{mg} \text { in frail } \\
\text { or elderly) for standard bronchoscopic procedures. } \\
\text { May be higher in longer procedures (e.g. EBUS) }\end{array}$ & $\begin{array}{l}\text { Onset of Sedation } \\
\text { Within } 2 \text { mins, with } \\
\text { maximum effect at 5-10 } \\
\text { mins. (May be longer in frail } \\
\text { or elderly or those with } \\
\text { chronic illnesses) } \\
\text { Duration of Action } \\
\text { Variable, but typical range is } \\
30-120 \text { mins } \\
\text { Approximate half-life } \\
1.5-2.5 \text { hours }\end{array}$ & $\begin{array}{l}\text { Respiratory depression, apnoea, bronchospasm, laryngospasm, } \\
\text { hypotension, heart rate alterations, cardiac arrest. } \\
\text { Life-threatening side effects and prolonged sedation are more } \\
\text { likely in the elderly and those with impaired respiratory or } \\
\text { cardiovascular status, hepatic impairment, renal impairment, } \\
\text { myasthenia gravis, and with rapid IV injection. }\end{array}$ & $\begin{array}{l}\text { Enhanced sedation and increased risk of respiratory } \\
\text { depression when combined with opioids. When } \\
\text { combined sedation is used, opioids should always be } \\
\text { administered prior to midazolam. } \\
\text { To prevent risk of accidental overdose, only } 1 \mathrm{mg} / \mathrm{mL} \\
\text { vials should be available in bronchoscopy suites. } 2 \mathrm{mg} / \\
\mathrm{mL} \text { or } 5 \mathrm{mg} / \mathrm{mL} \text { vials should not be available unless a } \\
\text { formal risk assessment has been undertaken. }\end{array}$ \\
\hline Fentanyl & $\begin{array}{l}\text { Slow IV injection - usually over } 1-3 \text { mins } \\
\quad \text { Initial dose: } 25 \text { micrograms } \\
\text { Supplemental doses, if required: } 25 \text { micrograms } \\
\text { Usual maximum total dose: } 50 \text { micrograms }\end{array}$ & $\begin{array}{l}\text { Onset of Sedation } \\
\text { Almost immediate, with } \\
\text { maximum effect at } 5 \text { mins } \\
\text { Duration of Action } \\
\text { Variable, but typical range is } \\
30-60 \text { mins } \\
\text { Approximate half-life } \\
\quad 2-7 \text { hours }\end{array}$ & $\begin{array}{l}\text { Nausea, vomiting and other GI upset, myoclonic movements, } \\
\text { respiratory depression, apnoea, bronchospasm, laryngospasm, } \\
\text { hypo/hypertension, arrhythmia, cardiac arrest. } \\
\text { Caution in elderly patients and those with impaired respiratory } \\
\text { or cardiovascular status, hepatic impairment and myasthenia } \\
\text { gravis. }\end{array}$ & $\begin{array}{l}\text { Enhanced sedation and respiratory depression when } \\
\text { given with benzodiazepines. When combined sedation } \\
\text { is used, opioids should always be administered prior to } \\
\text { midazolam. }\end{array}$ \\
\hline Alfentanil & $\begin{array}{l}\text { Slow IV injection - usually over } 30 \text { secs } \\
\text { Initial dose: } 250 \text { micrograms } \\
\text { Supplemental doses, if required: } 250 \text { micrograms } \\
\text { Usual maximum total dose: } 500 \text { micrograms }\end{array}$ & $\begin{array}{l}\text { Onset of Sedation } \\
\text { Almost immediate onset and } \\
\text { maximum effect } \\
\text { Duration of Action } \\
\text { Variable, but usually shorter } \\
\text { than fentanyl } \\
\text { Approximate half-life } \\
\quad 1-2 \text { hours }\end{array}$ & See Fentanyl & See Fentanyl \\
\hline Lidocaine & $\begin{array}{l}\text { Intranasal } \\
\text { Oropharnyx } \\
\text { Lidocaine } 2 \% \text { gel: } 6 \mathrm{~mL} \text { (120 mg) } \\
\text { Vocal cords, tracheobronchial tree } \\
\text { Lidocaine } 1 \% \text { solution: } 2 \mathrm{~mL} \text { boluses applied } \\
\text { topically, as required } \\
\text { Maximum total dose (see Table 3) } \\
\text { Use minimum dose to achieve effective cough } \\
\text { suppression and patient comfort. Subjective } \\
\text { symptoms of Lidocaine toxicity are common } \\
\text { when >9.6 mg/kg is used; much lower doses are } \\
\text { usually sufficient. }\end{array}$ & $\begin{array}{l}\text { Onset of Action } \\
\text { Duration of Action } \\
\text { Variable, but typical range is } \\
60-90 \text { mins } \\
\text { Approximate half-life } \\
\quad 1.5-2 \text { hours }\end{array}$ & $\begin{array}{l}\text { CNS effects (confusion, blurred vision, dizziness, drowsiness, } \\
\text { lightheadedness, myoclonus, nausea, nystagmus, paraesthesia, } \\
\text { restlessness, tremulousness, coma, convulsions, respiratory } \\
\text { failure) } \\
\text { CVS effects (hypotension, bradycardia, arrhythmia, cardiac } \\
\text { arrest). } \\
\text { Methaemoglobinaemia (rare). } \\
\text { Caution in those with hepatic and cardiac dysfunction, and with } \\
\text { significant renal impairment. }\end{array}$ & \\
\hline Adrenaline & $\begin{array}{l}\text { Topical } \\
\text { - Adrenaline 1:10,000: } 2 \text { to } 10 \mathrm{~mL}\end{array}$ & & Hypertension, tachycardia, arrhythmia, tremor. & \\
\hline
\end{tabular}


Table 2 Antagonists available for sedative drugs used in bronchoscopy

\begin{tabular}{|c|c|c|c|c|c|}
\hline Drug & \multicolumn{2}{|l|}{ Dose } & $\begin{array}{l}\text { Speed of Onset / } \\
\text { Duration of Action / } \\
\text { Half-life }\end{array}$ & Common / Serious Side Effects & Comments \\
\hline Flumazenil & \multicolumn{2}{|c|}{$\begin{array}{l}\text { To reverse midazolam } \\
\text { Initial dose: } 200 \\
\text { micrograms IV over } 15 \\
\text { secs } \\
\text { Supplemental doses: } \\
100 \text { micrograms every } \\
60 \text { secs if inadequate } \\
\text { response } \\
\text { Typical cumulative dose } \\
\text { range: } 300-600 \\
\text { micrograms } \\
\text { Maximum total dose: } \\
1 \text { mg }\end{array}$} & $\begin{array}{l}\text { Onset of Action } \\
1 \text { min } \\
\text { Duration of Action } \\
1-4 \text { hours } \\
\text { Approximate half-life } \\
\quad 40-80 \text { mins }\end{array}$ & $\begin{array}{l}\text { Nausea, vomiting, anxiety, agitation, dizziness, } \\
\text { hypertension, tachycardia. May lower seizure } \\
\text { threshold. May cause withdrawal in chronic } \\
\text { benzodiazepine users. }\end{array}$ & $\begin{array}{l}\text { Flumazenil has a shorter duration of action than } \\
\text { midazolam, and so patients should be observed long } \\
\text { enough to ensure that sedation and cardiorespiratory } \\
\text { depression does not recur once the effect of } \\
\text { flumazenil ceases. Further doses may be required. } \\
\text { Where combined sedation with midazolam and opioid } \\
\text { has been used, it is recommended that flumazenil is } \\
\text { administered first, unless a large dose of opioid has } \\
\text { been given }\end{array}$ \\
\hline Naloxone & \multicolumn{2}{|c|}{$\begin{array}{l}\text { To reverse opioids } \\
\text { Initial dose: } 100-200 \\
\text { micrograms IV } \\
\text { Supplemental dose: } 100 \\
\text { micrograms every } 2 \\
\text { mins if inadequate } \\
\text { response }\end{array}$} & $\begin{array}{l}\text { Onset of Action } \\
2-3 \text { mins } \\
\text { Duration of Action } \\
45 \text { mins to } \\
4 \text { hours } \\
\text { Approximate half-life } \\
1-1.5 \text { hours }\end{array}$ & $\begin{array}{l}\text { Nausea, vomiting, dizziness, headache, } \\
\text { tachycardia, hypo/hypertension. May cause } \\
\text { withdrawal in chronic opioid users. }\end{array}$ & $\begin{array}{l}\text { Naloxone has a shorter duration of action than many } \\
\text { opioids, and so patients should be observed long } \\
\text { enough to ensure that sedation and cardiorespiratory } \\
\text { depression does not recur once its effect ceases. } \\
\text { Further doses may be required. }\end{array}$ \\
\hline \multicolumn{6}{|c|}{$\begin{array}{l}\text { Adapted from 'Drugs in Bronchoscopy' (BTS Bronchoscopy eLearning Module available at: http://learninghub.brit-thoracic.org.uk/?bts=topic\&param=3), with kind permission of Toby } \\
\text { Capstick and Daniel G Peckham. }\end{array}$} \\
\hline Table 3 & \multicolumn{5}{|c|}{ Doses and concentrations of lidocaine used for bronchoscopy } \\
\hline \multicolumn{2}{|l|}{ Drug } & \multicolumn{2}{|c|}{ Dose per unit volume } & Site of application & Comments \\
\hline \multicolumn{2}{|c|}{ Lidocaine $2 \%$ gel } & \multicolumn{2}{|c|}{$20 \mathrm{mg} / \mathrm{mL}$} & Nasal & Gel preparation syringe typically contains $6 \mathrm{~mL}(120 \mathrm{mg}$ ) \\
\hline \multicolumn{2}{|c|}{ Lidocaine $10 \%$ aerosol spray } & \multicolumn{2}{|c|}{$10 \mathrm{mg} /$ actuation } & Oropharynx & 3 actuations $(30 \mathrm{mg}$ ) often sufficient \\
\hline \multicolumn{2}{|c|}{ Lidocaine $1 \%$ solution } & \multicolumn{2}{|c|}{$10 \mathrm{mg} / \mathrm{mL}$} & Vocal cords, trachea and bronchial tree & \\
\hline
\end{tabular}

\section{APPENDIX 9 SEDATION SCORING SCALES WHICH MAY BE USED TO AID ASSESSMENT AND DOCUMENTATION OF SEDATION LEVEL}

\begin{tabular}{ll}
\hline Ramsay Scale (RS) & Response \\
\hline Level & Anxious and agitated or restless \\
\hline 1 & Cooperative, orientated and tranquil \\
2 & Responds only to commands \\
3 & Brisk response to light glabellar touch or loud noise \\
4 & Sluggish response to light glabellar touch or loud noise \\
5 & No response to light glabellar touch or loud noise \\
6 & Ramsay MA, Savege $T M$, Simpson BR, Goodwin R. Controlled sedation with \\
alphaxalone-alphadolone. Br Med J. 1974 Jun. 22;2(5920):656-9.
\end{tabular}

\begin{tabular}{ll}
\begin{tabular}{l} 
Modified Observer's Assessment of Alertness/Sedation (MOAAS) scale \\
\hline Level
\end{tabular} \\
\hline 5 & $\begin{array}{l}\text { Responds readily to name spoken in normal tone } \\
\text { Lethargic response to name spoken in normal tone }\end{array}$ \\
4 & Responds only after name is called loudly or repeatedly \\
3 & Responds only after mild prodding or shaking \\
2 & Does not respond to mild prodding or shaking \\
1 & Does not respond to pain \\
0 & \\
\hline $\begin{array}{l}\text { Chernik DA, Gillings D, Laine H, Hendier J, Silver JM, Davidson AB, et al. Validity and } \\
\text { reliability of the Observer's Assessment of Alertness/Sedation Scale: study with } \\
\text { intravenous midazolam. J Clin Psychopharmacol. 1990 Aug.; 10(4):244-51. }\end{array}$
\end{tabular}




\section{APPENDIX 10 BTS FLEXIBLE BRONCHOSCOPY - SUGGESTED GUIDES ON HOW TO PERFORM STANDARD PROCEDURES}

\section{BRONCHOALVEOLAR LAVAGE}

This procedure is performed during flexible bronchoscopy and is useful in the diagnosis of pulmonary infections and may be useful in the diagnosis of parenchymal lung disease.

The site of BAL should be guided by available radiology.

i. Once the site for BAL has been chosen, the bronchoscope should be advanced until the desired site is reached.

ii. The bronchoscope should then be wedged in to a position where the lumen of the bronchus is occluded by the bronchoscope

iii. Whilst the bronchoscope is in this position, $60-180 \mathrm{mls}$ of normal saline should be instilled in to the segment

iv. Low-pressure suction should then be applied to retrieve the sample. The aim should be to apply enough suction to retrieve the sample without causing airway collapse.

v. To ensure an adequate sample is retrieved it is useful to have tubing with several containers in series attached to the suction pump.

vi. Some centres employ a syringe to retrieve the sample from the same port that the saline is instilled through.

\section{ENDOBRONCHIAL BIOPSY}

This procedure is performed for visible airway abnormalities, most commonly in the diagnosis of suspected lung cancer. Once again, radiology should be available to plan where sampling should occur. There are several types of biopsy forceps, most commonly alligator or open cup forceps are used. Studies have not demonstrated any advantage of one type of forceps over the other in diagnostic yield.

i. The bronchoscope should be advanced until the endobronchial lesion is visualised.

ii. Secure the bronchoscope in this position so that the abnormality can be fully visualised, then advance the forceps in the closed position through the working channel of the bronchoscope.

iii. Once the forceps are visualised at the distal end of the bronchoscope the forceps can be opened.

iv. Whilst keeping the bronchoscope still the forceps should be advanced in the open position towards the abnormality.

v. Once the target is reached the forceps should be closed trapping as much tissue as possible.

vi. The forceps should be retracted and removed from the working channel of the bronchoscope.

vii. A "tugging" sensation may be felt whilst retracting the forceps.

viii. Once the sample has been removed from the forceps, it should be reinserted into the working channel and the procedure repeated $5-6$ times.

\section{ENDOBRONCHIAL BRUSH}

This procedure is performed to gain cytology samples in areas of abnormal mucosa or endobronchial lesions. Although no evidence exists for which procedure is performed first we suggest that endobronchial biopsies are performed prior to brushings.

i. Once the area of abnormality is detected the bronchial brush is inserted through the working channel of the bronchoscope in its retracted position in its protective sheath

ii. Once the bronchial brush is seen at the distal end of the bronchoscope the brush can be pushed out (opened)

iii. The brush is then advanced towards the lesion and moved back and forth over the lesion several times

iv. The brush is then retracted into its sheath (closed) and removed from the working channel

V. The procedure is repeated

\section{TRANSBRONCHIAL LUNG BIOPSY}

This procedure is performed via flexible bronchoscopy, to aid the diagnosis of parenchymal lung disease. Unlike endobronchial biopsy this procedure can be performed "blind", without direct visualisation of the lesion. Some centres chose to perform this procedure with radiological guidance (fluoroscopy), diagnostic yield does not differ between methods. Accurate radiological imaging is essential to guide the bronchoscopist to the lung parenchyma with the greatest potential diagnostic yield in non-diffuse interstitial disease.

i. The bronchoscope is advanced as far as possible into the area of the lung to be biopsied

ii. The forceps are then inserted into the working channel and advanced as far as possible

iii. At this point the forceps are retracted $1 \mathrm{~cm}$ to avoid a biopsy of the pleura Instruct the patient to take a slow deep breath in. The forceps are then opened during inspiration

iv. Instruct the patient to breathe out slowly, whilst the patient is breathing out the forceps are advanced and closed

v. The forceps are then removed and the sample retrievedWarning: Do not take the biopsy if the patient experience pain when forceps is pulled back or removed, pleura could have been caught in the forceps. Open forceps and remove without biopsy.

vi. The procedure is repeated a further 5-6 timesClinical Tip: Whilst performing a TBLB co-operation of the patient is necessary. Patients should be able to follow commands and light sedation is therefore advised. 
This is electronically available on the BTS website to download and print

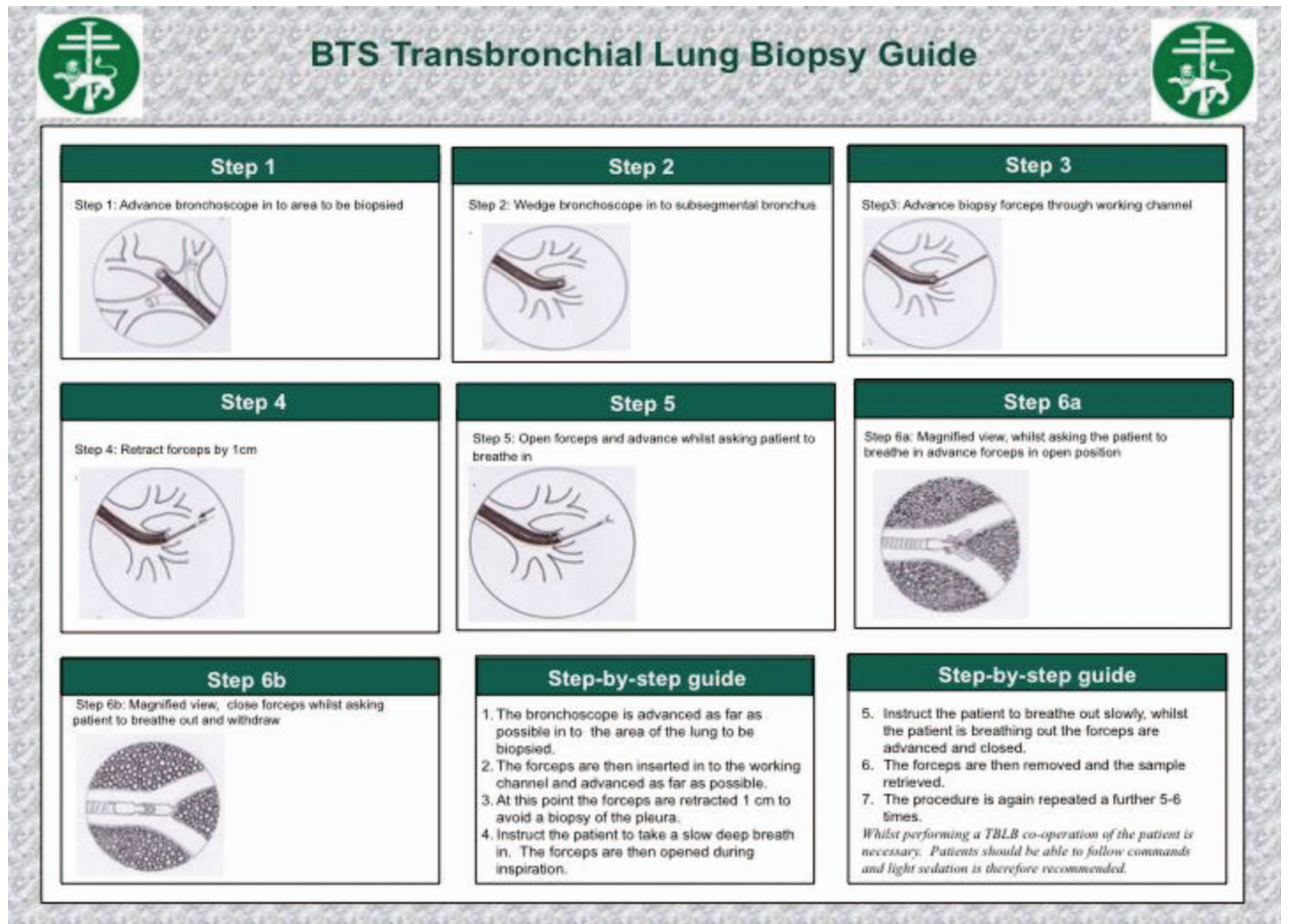

APPENDIX 11 RECOMMENDATIONS FOR SIZE OF BRONCHOSCOPE WITH DIFFERENT AIRWAY DEVICES

\begin{tabular}{lc} 
& \\
\hline Airway device size & New scope sizes \\
\hline ETT 3.5 & $<2.7$ \\
ETT 5.5 & 2.7 \\
ETT 6.5 & 2.7 \\
ETT 8.0 & 5.3 \\
ETT 9.0 & 7.3 \\
ETT 10.5 & 8.7 \\
\hline ETT- Endotracheal Tube & \\
Table adapted from: & \\
Brimacombe J, Dunbar-Reid K. The effect of introducing fibreoptic bronchoscopes on \\
gas flow in laryngeal masks and tracheal tubes. Anaesthesia. 1996 Oct; $51(10): 923-8$
\end{tabular}




\section{APPENDIX 12: CLEANING AND DISINFECTING PROCEDURE}

\section{Wear appropriate, protective clothing}

(Gloves, aprons, visors or goggles, face mask and forearm covers)

1 Before removing bronchoscope from light source or video processor suction with enzymatic detergent, followed by 10 seconds of air. This will remove blood, mucus and debris from internal channels. Wipe the external surface of the scope with a soft lint free cloth soaked in detergent. Check scope for damage. Remove, flush and wipe biopsy and suction ports. Discard single use valves.

2 Transport bronchoscope to the decontamination area in an appropriately sized and covered receptacle.

3 Leak test bronchoscope according to manufacturer's recommendations.

4 Manual cleaning of bronchoscope to be performed in a dedicated sink filled with water and detergent according to manufacturer's recommendations. Water and detergent to be discarded after each use.

5 Brush suction and biopsy channels and ports with a single-use brush, according to manufacturer's guidelines. Ensure the brush is visibly clean at the end of the process.

6 Clean the external surface of the scope, around the angulation control and distal tip of the bronchoscope.

7 Brush the biopsy and suction channel ports with a single-use short brush.

8 Irrigate the channels with an enzymatic detergent followed by water, then air.

9 Transfer the bronchoscope to a separate sink for rinsing, to remove residual detergent.

10 Transfer bronchoscope to AER in an appropriately sized receptacle.

11 Disinfect in Automated Endoscope Reprocessor alongside reusable valves. Ensure all channels are exposed to disinfectant process.

12 Store hanging in cupboard or drying cabinet following manufacturers recommendations.

13 Maintain record of decontamination process for bronchoscope and accessories. 


\section{APPENDIX 13: PATIENT INFORMATION LEAFLET}

This is electronically available on the BTS website to download and print

\section{Can anything go wrong?}

Bronchoscopy is a safe procedure with little risk and complications are relatively rare. There is a small risk of infection and bleeding associated with the procedure, but every effort is made to prevent this from happening. Air can also sometimes leak into the space surrounding your lung during the procedure but this is not usually a problem and the air can be taken out with a needle and syringe or in some instances a drain (little plastic tube) needs to be inserted and left inside for a couple of hours to drain the air out.

Occasionally patients take a longer time to recover after the procedure and may need to be admitted to hospital for observation.

\section{What happens after the bronchoscopy?}

You will be looked after by a nurse until you are awake enough to leave. Your breathing rate, pulse and blood pressure will be checked. You may have a chest X-ray. You may cough up small amounts of blood: inform the nurse if you cough up more than a tablespoon of blood, have chest pain or difficulty breathing. You should not eat or drink until the feeling in the back of your throat has returned, usually 2 hours.

It is common to experience a mild sore throat, hoarseness and cough after the procedure. It is important that you do not drive a car or operate machinery if you had sedation (medication in the vein to make you sleepy).

In some cases the doctor can give you some initial results of your bronchoscopy when you are awake before you leave.

\begin{tabular}{|l|}
\hline Important aspects of bronchoscopy \\
\hline$\quad$ Do not eat anything for 4 hours or drink liquids for 2 hours before your procedure \\
\hline Inform your doctor of all the medication you take and any medical conditions. \\
\hline $\begin{array}{l}\text { Arrange for someone to take you to and fetch you home after the bronchoscopy. It } \\
\text { may be necessary for someone to stay with you for a couple of hours after the proce- } \\
\text { dure if you live by yourself. }\end{array}$ \\
\hline $\begin{array}{l}\text { Do not eat or drink after the procedure until the numbness in your mouth/throat has } \\
\text { completely worn off. }\end{array}$ \\
\hline $\begin{array}{l}\text { For } 24 \text { hours after the bronchoscopy you can not drive, return to work, operate machin- } \\
\text { ery, drink alcohol, sign legal documents or be responsible for small children. }\end{array}$ \\
\hline $\begin{array}{l}\text { Contact your doctor if you are short of breath, have chest pain or cough up more than a } \\
\text { tablespoon of blood. }\end{array}$ \\
\hline $\begin{array}{l}\text { Contact phone number: } \\
\text { Appointment Date: } \\
\text { Appointment Time: }\end{array}$ \\
\hline
\end{tabular}

What is a bronchoscopy?

A bronchoscopy is an examination of the breathing passages/tubes (airways) of your lungs. The bronchoscopy is done with a thin tube-like instrument with a mini camera at its tip, called a bronchoscope (see picture). The bronchoscope enables your doctor to see inside the breathing passages of your lungs and if needed take samples of mucus (phlegm) or tissue from inside your lungs.

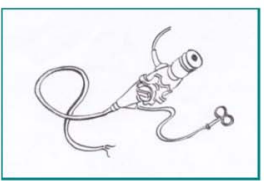

A bronchoscope

Why do I need a bronchoscopy?

Some of the common reasons why bronchoscopies are done include:

- Infection, specific samples from your lungs can help your doctor to find the causes of infections and give you appropriate treatment. Your doctor can also use the bronchoscope to clear some of the mucus from your breathing passages.

- Bleeding, your doctor can look inside your breathing passages to determine where the blood is coming from that you are coughing up.

- An abnormal Chest X-ray, there may be a narrowing of a breathing passage or a "spot" on your lung that your doctor wants to investigate with the bronchoscope and take small samples to find the cause.

- Persistent cough, samples taken during a bronchoscopy and an examination of the breathing passages can sometimes help to determine the cause for a cough that does not respond to usual medication.

- Noisy breathing, a bronchoscopy can help to see if narrowing of your breathing passages or erratic movement of your vocal cords (voice box) is causing additional breathing sounds.

Your doctor will explain why you need the bronchoscopy.

\section{(9) \\ British Thoracic Society \\ Bronchoscopy \\ Patient Information Leaflet}

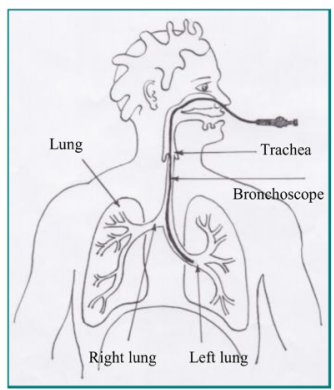

This leaflet explains the procedure called a BRONCHOSCOPY it explains why we do bronchoscopies and what you can expect if you are having the procedure done. It also answers the most frequently asked questions about having a bronchoscopy.

If you have any further questions, please do not hesitate to ask a member of your medical team.
Is there an alternative test that I can have instead of the bronchoscopy? Your doctor can do breathing tests, X-rays and CT-scans of your lungs. These tests will give additional information about your lungs. A bronchoscopy gives very specific information to your doctor by looking inside your breathing passages and obtaining specific samples.

How do I prepare for my bronchoscopy?

You will need to provide an up-to-date list of all your medication, allergies and any medical conditions. Your doctor will review this with you. It is important to let your doctor know if you are taking any blood thinning medication like Warfarin/Asprin etc or if you are diabetic. You can not eat for 4 hours or drink anything for 2 hours before the procedure. It is better not to smoke before the procedure.

What will happen before the procedure?

The procedure will be explained to you again and you will have the opportunity to ask any questions. You will be asked to sign a consent form. In the room where you will have your bronchoscopy the oxygen levels in you blood and your heart rate is monitored with a probe (loose clip) on you finger. A cannula (tiny plastic tube) is inserted in one of your veins and you may be given medication in the vein to make you feel sleepy. You may be given some oxygen.

What happens during a bronchoscopy?

A local anaesthetic will be applied to your nose and the back of your throat This can be uncomfortable; the anaesthetic does not taste very good and you may have a sensation of numb 'blocking' in the back of your throat. It feels as if you can't swallow, but you can: the back of your throat is just temporarily numb. Your doctor will then insert the bronchoscope via your nose/mouth to the back of your throat to numb your voice box with local anaesthetic. While your voice box and breathing passages are being numbed, you may cough: this usually settles down when the local anaesthetic takes effect. The coughing may however cause you some discomfort during the procedure. The doctor will look inside the breathing passages and take samples; you may be offered more anaesthetic or sedation to help address any discomfort you may experience. 
